\title{
Neural coding of
}

\section{grasp force planning and \\ control in macaque areas \\ AIP, F5, and M1}

\author{
Dissertation \\ for the award of the degree \\ "Doctor rerum naturalium" \\ of the Georg-August-Universität Göttingen
}

within the doctoral program Center for Systems Neuroscience

of the Georg-August University School of Science (GAUSS)

\author{
submitted by \\ Rijk W. in 't Veld
}

from Utrecht, The Netherlands

Göttingen, 2016 


\section{Doctoral thesis committee}

Referee: $\quad$ Prof. Dr. Hansjörg Scherberger

Research Group Neurobiology

German Primate Center

Kellnerweg 4

37077 Göttingen

Co-referee: $\quad$ Prof. Dr. Alexander Gail

Sensorimotor Group

German Primate Center

Kellnerweg 4

37077 Göttingen

Dr. Igor Kagan

Decision and Awareness Group

German Primate Center

Kellnerweg 4

37077 Göttingen

External members of the examination board

Prof. Dr. Andrea Antal

Göttingen University Medical School

Dept. of Clinical Neurophysiology

Robert-Koch-Straße 40

37075 Göttingen

Germany

Prof. Dr. Dario Farina

University Medical Center Göttingen

Dept. of Neurorehabilitation Engineering

Von-Siebold-Straße 4

37075 Göttingen

Germany

Prof. Dr. Ralf Heinrich

Dept. Cellular Neurobiology

Schwann-Schleiden Research Centre

Julia-Lermontowa-Weg 3

37077 Göttingen

Germany

Date of oral examination: September 20 ${ }^{\text {th }}, 2016$ 
Herewith I declare that I have written this thesis independently and with no other aids and sources than quoted.

Göttingen, June $20^{\text {th }}, 2016$ Rijk W. in 't Veld 





\section{Acknowledgements}

There are many people I need to thank for helping me putting this work together. First of all, I want to thank my supervisor, Hans Scherberger. He took me into his lab about four years ago and has supported me since the first day. He was always easy to approach for questions and I think that it mainly is thanks to his relaxed and friendly character that we always had such a great atmosphere in the lab. He has also taught me much about science and provided me with important feedback that helped me improve whatever work I produced. For this, I also want to thank my other thesis committee members, Alexander Gail and Igor Kagan. I could always count on receiving useful comments from them in our annual meetings and on other occasions.

I also thank all my former teachers and supervisors who all contributed to teaching me essential knowledge and skills that are required in science. Especially Richard van Wezel, who introduced me to the world of primate neurophysiology, and Nicholas Hatsopoulos, who introduced me to the field of motor neuroscience.

Great thanks goes out to all my colleagues in the Neurobiology Laboratory, both former and current. Not just for their support during the countless hours in the lab, but also for all the fun activities like movie nights, barbecues, parties, games, and sport we did outside the lab. I thank you Matthias, Natalie, Ricarda, Sabine, Anja, Sebastian, Steve, Yves, Stefan S, 
Katharina, Ben, Jonathan, Wei-An, Andres, Jeroen, Daniela, Anne-Do, Swathi, Stefan G, Andrej, Lukas, Megha, and all the other students who spent some time in our lab over the last years.

Special thanks goes out to Matthias Dörge for his invaluable support in designing, building and repairing the experimental setup. I especially appreciate the speed and easiness of how he got things done, most requests were followed by a "Ja, I will do that", and it was done a few hours later. Also special thanks to Natalie Nazarenus for her assistance in animal training, all her lab managing, and for her unorthodox attitude. She not only made the lab run smoothly, but also a fun place to work. Great thanks as well to Benjamin Dann and Jonathan Michaels for teaching me so much relevant knowledge and for proofreading parts of this thesis, but also for the many hours of interesting work-related and non-work-related discussions.

Much respect goes to the two monkeys I worked with, Sally and Bart. Without their contributions none of this would have been possible. I am also very grateful for the work of the animal caretakers and veterinarians who took care of them and the other monkeys.

Besides all my lab colleagues, there are also many other people in Göttingen to thank for the great time I had outside of work and for helping me improving my German. First of all, my roommates of the Gotmar-Kommune and the Zimmermannstraße who made me feel at home here in this weird country. But also the people from the Leichtathletik and Kickboxen 
courses at the Uni-Sportzentrum. And of course all the other employees of the German Primate Center that I have had the pleasure of meeting.

Thanks to all my friends and family in the Netherlands as well. It was always great to go back home and have so many people I can meet and spend some quiet or exciting time with. I also loved it whenever they came to visit me in Göttingen. Thanks to Jeff and Alain, who I could often meet online. And great thanks to my parents and siblings, Wim, Rinske, Tess, and Niek, for all the support and love that I have received all my life. 


\section{Table of contents}

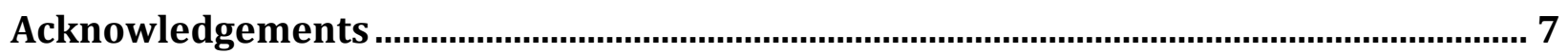

Table of contents.....................................................................................................

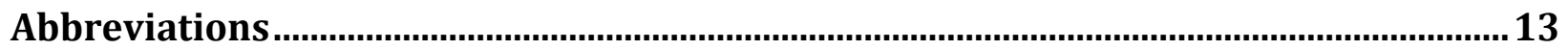

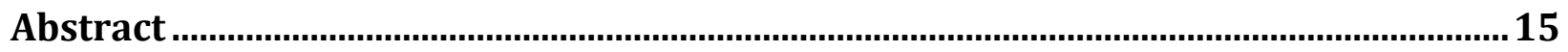

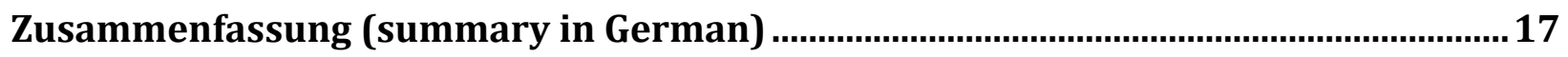

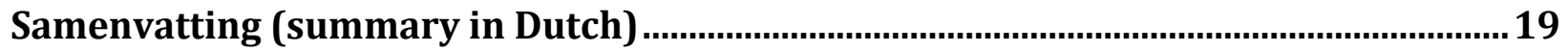

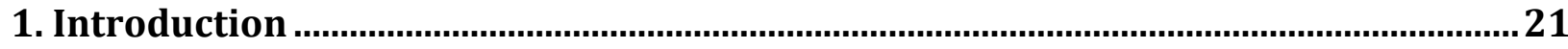

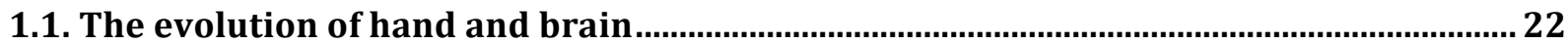

1.1.1. Origin of the hand ........................................................................................................................22

1.1.2. The brain behind the hand …………………………………………………………………………….....23

1.2. Visual guidance of grasping ....................................................................................... 26

1.2.1. Visual information pathways …………………………………………………………………….22

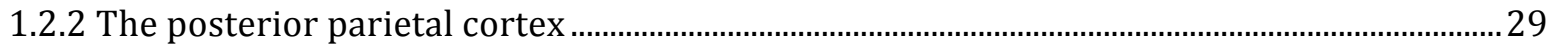

1.3. Cortical grasping network

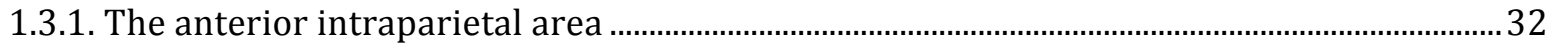

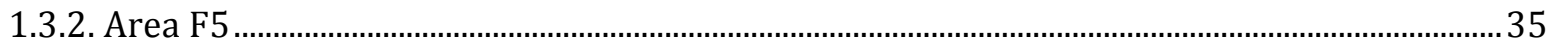

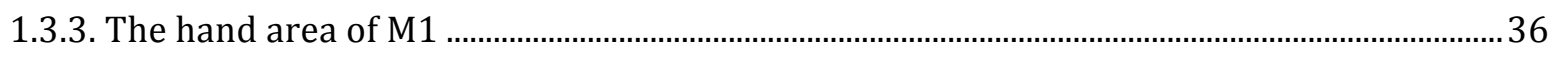

1.4. Force coding in the cortical grasping network .................................................................. 38

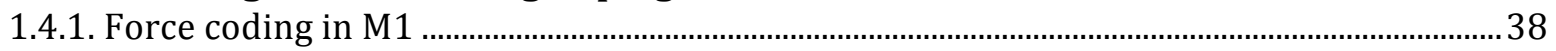

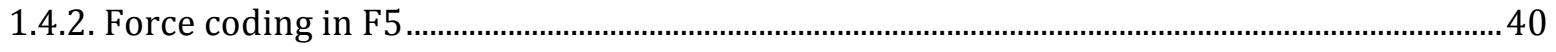

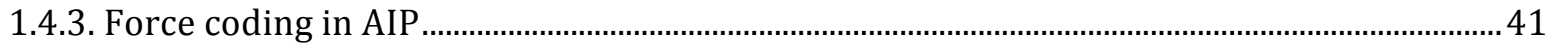

1.5. Motivation for this study .............................................................................................. 44

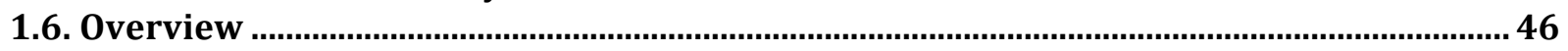

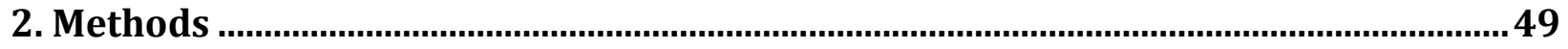

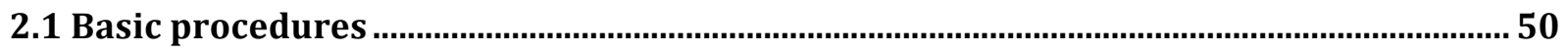

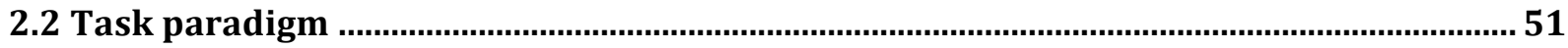

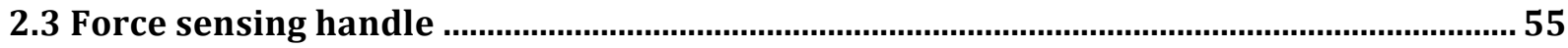

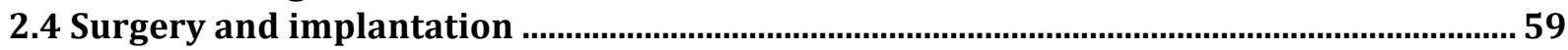

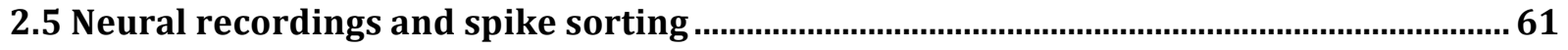

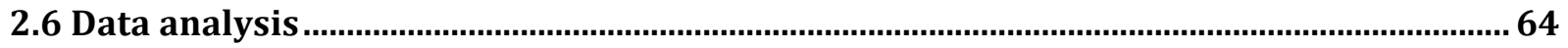

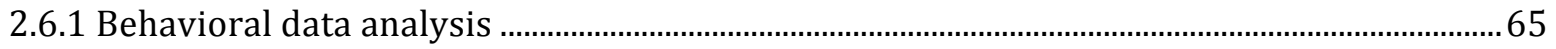

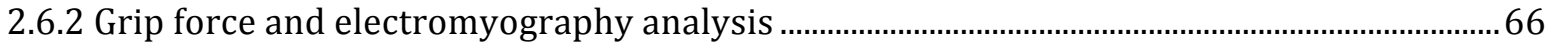

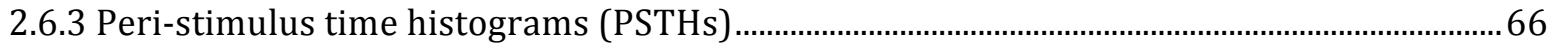

2.6.4 Cluster-based permutation test ............................................................................................................6.

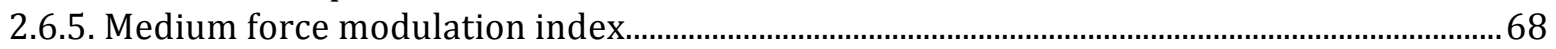

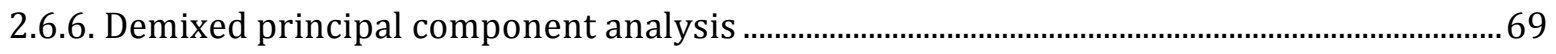

2.6.7 Best-channel set........................................................................................................................

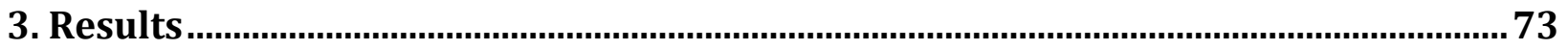

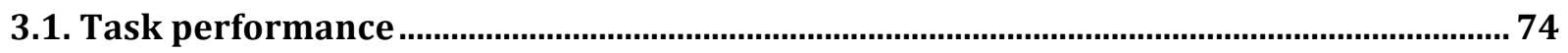

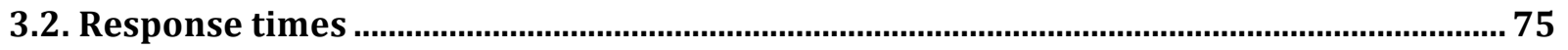

3.3. Force profiles.......................................................................................................... 76

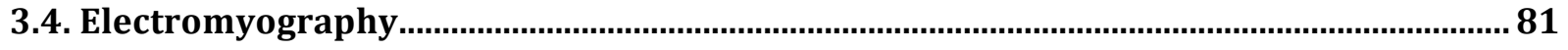




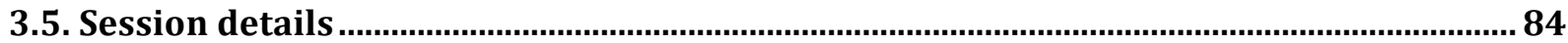

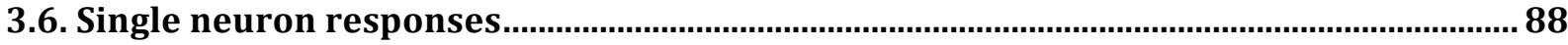

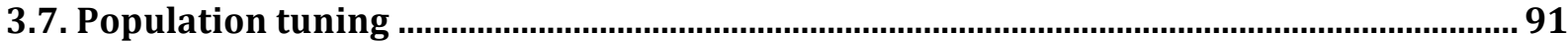

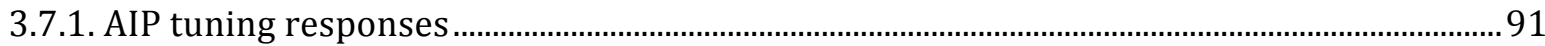

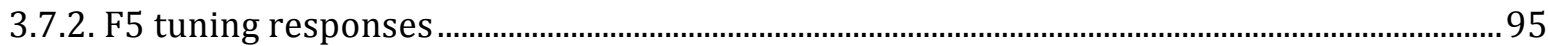

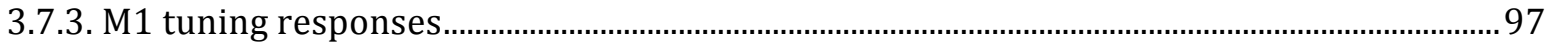

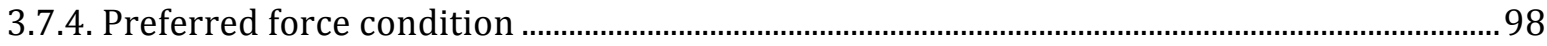

3.7.5. Comparing best-channel set to average tuning.................................................................... 104

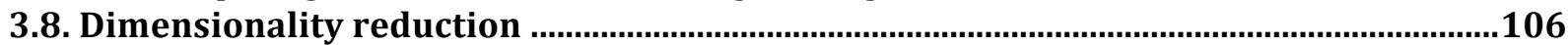

3.8.1. Demixed components and explained variance in AIP ....................................................... 109

3.8.2. Demixed components and explained variance in F5 ........................................................... 115

3.8.3. Demixed components and explained variance in M1 .............................................................. 119

3.8.4. Response of demixed components to medium- and high force ........................................... 121

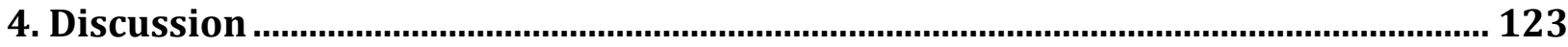

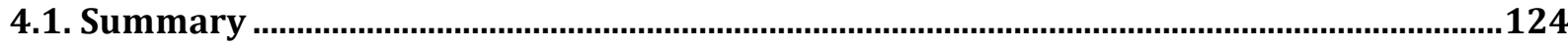

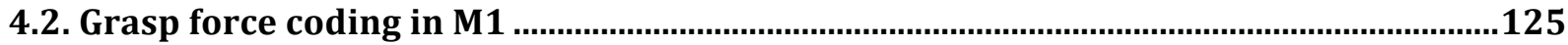

4.3. Grasp force coding in F5

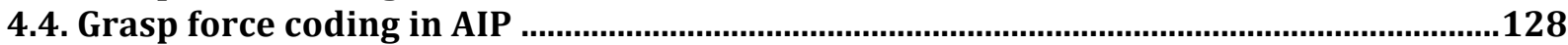

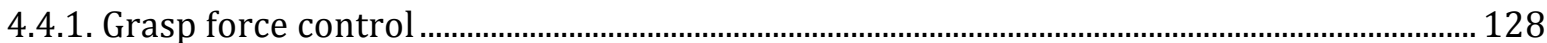

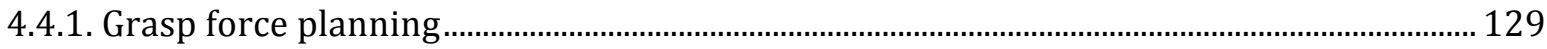

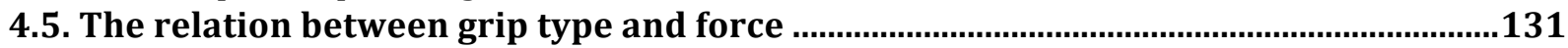

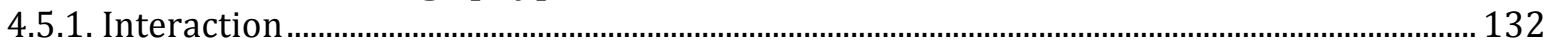

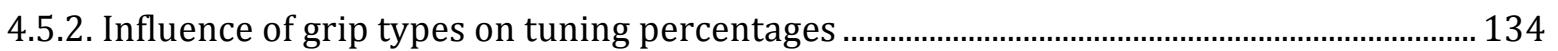

4.5.3. Temporal difference between grip type and force coding ...................................................... 135

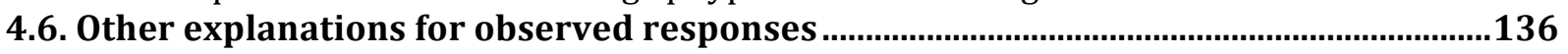

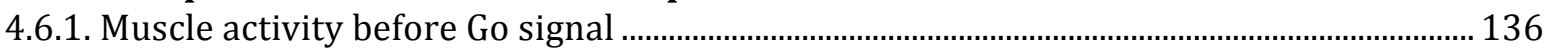

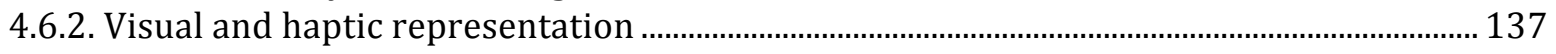

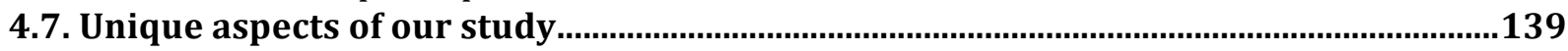

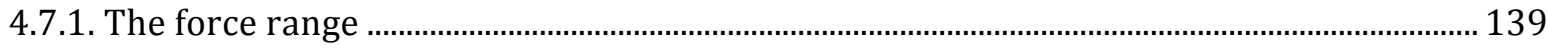

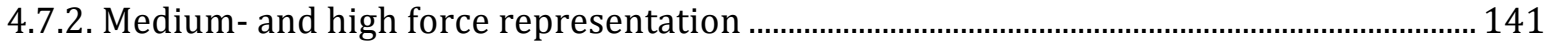

4.8. Functional role of AIP, F5, and M1 in grasp force control .................................................141

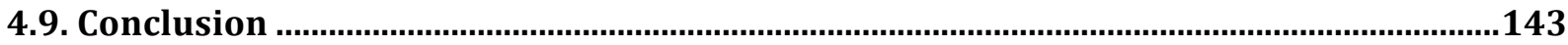

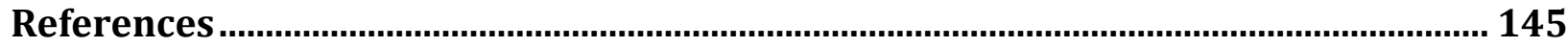

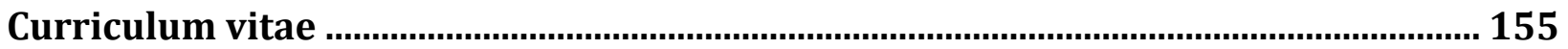

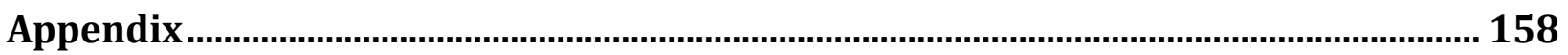




\section{Abbreviations}

$\mathrm{AIP}=$ Anterior intraparietal area

$\mathrm{AIT}=$ Anterior inferotemporal cortex

BOLD = Blood oxygen level-dependent

$\mathrm{dPCA}=$ Demixed principal component analysis

EMG= Electromyography

F5= Rostral part of the ventral premotor area

$\mathrm{fMRI}=$ functional magnetic resonance imaging

LIP= Lateral intraparietal cortex

M1= Primary motor cortex

MT= Middle temporal area

PHF $=$ Precision grip High Force (task condition)

PLF $=$ Precision grip Low Force (task condition)

$\mathrm{PMC}=$ Premotor cortex

$\mathrm{PMF}=$ Precision grip Medium Force (task condition)

$\mathrm{PMv}=$ Ventral premotor area

$\mathrm{PPC}=$ Posterior parietal cortex

PSTH $=$ Peristimulus time histogram

TMS= Transcranial magnetic stimulation

V1= Primary visual cortex

WHF $=$ Whole-hand grip High Force (task condition) 
WLF = Whole-hand grip Low Force (task condition)

WMF $=$ Whole-hand grip Medium Force (task condition) 


\section{Abstract}

Much research has been done in the last decades to decipher how the brain controls grasping movements. The anterior intraparietal area (AIP), the hand area of the ventral premotor cortex (F5), and the hand area of the primary motor cortex (M1) have been identified as essential cortical areas for the control of hand shape. However, much less is known about how neurons from these areas code another essential parameter of grasping actions, grasp force. Especially, the role of the higher order areas F5 and AIP in this process remains elusive. This study aims to address the lack of knowledge about the neural coding of grasp force planning and control in these areas.

To achieve this, we trained two macaque monkeys (Macaca mulatta) on a delayed grasping task with two grip types (whole-hand grip or precision grip) and three different levels of force $(0-12 \mathrm{~N})$. While the monkeys performed the task, we recorded the activity of singleand multi-units from AIP, F5, and M1. We calculated the percentage of grip type and force tuned units (cluster-based permutation test) and calculated the amount of variance explained by grip type and force for the population of units of each brain area (demixed principal component analysis).

We show here, for the first time, the modulation of single AIP neurons to grasp force. Furthermore, we confirm and extend previous findings that showed such neural modulation in F5 and M1. Surprisingly, the percentages of units responding to grasp force control in AIP and F5 were not much less than M1 and similar to the amount of units responding to grip type. In F5, the amount of variance explained by grasp force was almost 
as high as that explained by grip type. In AIP and M1, grip type clearly explained more variance than grasp force, but also in these areas, the amount of variance explained by force was sufficient to reliably decode the force conditions. We also found strong neural modulation to grasp force conditions before movement onset in F5, which possibly represents a role of this area for grasp force planning. In AIP, grasp force planning activity was found only in one of both monkeys, and as expected, not in M1 (checked only in one animal). Lastly, we found that, although force tuning was influenced by grip type in some units, only a small fraction of the population variance in each area contained mixed selectivity for grip type and force. Information about grasp force could therefore be extracted separately from grip type.

These findings suggest an important role of AIP and F5 in grasp force control in addition to M1. F5 is likely also involved in planning grasp force, while the role of AIP and M1 are likely smaller in this process. Finally, since grip type and force information could be extracted separately, these results show that grasp force is possibly coded independently from hand shape in the cortical grasping network. 


\section{Zusammenfassung (summary in German)}

Thanks to Benjamin Dann for helping with the translation.

In den letzte Jahrzehnten wurde viel daran geforscht zu entschlüsseln wie das Gehirn Greifbewegungen koordiniert. Das anteriore intraparietale Areal (AIP), das Hand Areal des ventralen premotorischen Kortex (F5), und das Hand Areal des primären motorischen Kortex (M1) wurden als essentielle kortikale Arealen für die Kontrolle der Hand identifiziert. Nichtsdestotrotz ist deutlich weniger darüber bekannt wie die Neuronen dieser Areale einen weiteren essentielle Parameter von Greifbewegungen kodieren: Greifkraft. Insbesondere die Rolle der tertiären, kortikalen Areale AIP und F5 in diesen Prozess ist bisher unklar. Die hier durchgeführte Studie befasst sich mit der Wissenslücke über die neuronale Kodierung von Greifkraft Planung und Steuerung in diesen Arealen.

Um dies zu erreichen, haben wir zwei Makaken (Macaca mulatta) trainiert eine verzögerte Greifaufgabe auszuführen mit zwei Grifftypen (ein Griff mit der ganzen Hand oder ein Präzisionsgriff) und mit drei verschiedene Kraftniveaus (0-12 N). Während die Affen die Aufgabe ausführten, haben wir die Aktivität von "single-units" (einzelnen Neuronen) und "multi-units" (Gruppen von mehreren Neuronen) in den Arealen AIP, F5 und M1 aufgenommen. Wir berechneten den Prozentsatz von Grifftyp modulierten und Griffkraft modulierten "units" (cluster-based permutation test) und berechneten wie viel Varianz in der Population von "units" durch Grifftyp und Kraft erklärbar ist, separat für jedes Gehirn Areal mit einer modernen Dimensionalitätsreduktionsanalyse (demixed principal component analysis). 
Wir zeigen hier zum ersten Mal die Modulation von einzelnen AIP Neuronen durch Greifkraft. Weiterhin bestätigen und erweitern wir hier vorherige Ergebnisse, welche solche neuronale Modulationen bereits in F5 und M1 gezeigt haben. Überaschenderweise war der Prozentsatz von "units" welche durch Griffkraft moduliert werden, in AIP und F5 nicht wesentlich kleiner als in M1 und ähnlich zu dem Prozentsatz an Grifftyp modulierte Neuronen. Der Anteil an erklärte Varianz in F5 durch Greifkraft war nahezu so groß, wie der Anteil erklärt durch Grifftyp. In AIP und M1 war klar mehr Varianz durch Grifftyp erklärt als durch Kraft, aber der Anteil an erklärte Varianz beider Arealen war ausreichend, um zuverlässig Kraftbedingung zu dekodieren. Wir fanden ebenfalls eine starke neuronale Modulation für Griffkraftbedingungen vor der Bewegungsinitiierung in F5, was wahrscheinlich eine Rolle dieses Areals in der Greifkraftplanung repräsentiert. In AIP war Greifkraftplanungsaktivität nur in einen der beiden Affen vorhanden und wie erwartet nicht präsent in M1 (gemessen nur in einen Affen). Letztendlich, obwohl Greifkraftmodulation in einigen Fällen durch Grifftypmodulation beeinflusst war, war nur ein kleiner Anteil der Populationsvarianz, in den jeweiligen Arealen, durch interaktive Modulation erklärt. Information über Greifkraft können somit folglich separat vom Grifftyp extrahiert werden.

Diese Ergebnisse legen eine wichtige Rolle von AIP und F5 bei der Greifkraftkontrolle, neben M1, nah. F5 ist mit hoher Wahrscheinlichkeit auch bei der Planung von Greifkraft involviert, während die Rolle von AIP und M1 geringer ist in diesem Prozess. Letztendlich, da Grifftyp- und Kraftinformation separat extrahierbar sind, zeigen diese Ergebnisse, dass Greifkraft vermutlich unabhängig von Grifftyp, im kortikalen Greifnetzwerk kodiert ist. 


\section{Samenvatting (summary in Dutch)}

In de laatste decennia is er veel onderzoek gedaan om te interpreteren hoe de hersenen grijpbewegingen besturen. Het anterieure intra pariëtale gebied (AIP), het handgebied van de ventrale premotorische schors (F5) en het handgebied van de primaire motorische schors (M1) zijn geïdentificeerd als essentiële gebieden van de hersenschors die de vorm van de hand besturen. Maar er is veel minder bekend over hoe de hersenen een andere parameter van grijpbewegingen bestuurt: grijpkracht. Vooral de rol in dit proces van AIP en F5, gebieden van hogere orde, is nog nagenoeg onbekend. Deze studie richt zich op het gebrek aan kennis over de neurale codering van het plannen en besturen van grijpkracht.

Om dit te bereiken, hebben we twee makaken (Macaca mulatta) getraind om een vertraagde grijptaak uit te voeren met twee grepen van de hand (een grip met de hele hand of een precisie grip) en met drie verschillende krachtniveaus (0-12 N). Terwijl de apen de taak uitvoerden, maten we de activiteit van single-units (individuele neuronen) en multiunits (collectie van enkele neuronen) in de gebieden AIP, F5 en M1. We berekenden het percentage van units die hun activiteit moduleerden op basis van grip vorm of kracht met een moderne statistieke test (cluster-based permutation test) en we berekenden de hoeveelheid variantie die werd verklaard door de grip vorm en kracht door de populatie van units van elk hersengebied met een moderne dimensie vermindering techniek (demixed principal component analysis).

We laten hier voor het eerst zien dat individuele neuronen van AIP hun activiteit moduleren op basis van grijpkracht. Verder bevestigen we dat neuronen van F5 en M1 
dergelijke modulaties vertonen en breiden we de kennis hierover uit. Verassend genoeg was het percentage units dat reageert op het besturen van grijpkracht in AIP en F5 niet veel lager dan in M1 en ongeveer gelijk aan de hoeveelheid units dat reageert op grip vorm. De hoeveelheid variantie die werd verklaard door grijpkracht in F5 was bijna net zo hoog als wat werd verklaard door grip vorm. In AIP en M1 verklaarde grip vorm duidelijk meer variantie dan grijpkracht, maar ook in deze gebieden was de hoeveelheid variantie dat grijpkracht verklaarde hoog genoeg om de kracht conditie te decoderen. We vonden ook een sterke neurale modulatie voor grijpkracht condities in F5 voordat de arm bewoog, wat mogelijk een rol voor dit gebied representeert in het plannen van grijpkracht. In AIP was activiteit voor het plannen van grijpkracht alleen in één van beide apen gevonden en zoals verwacht was het niet gevonden in M1 (onderzocht in één aap). Tenslotte vonden we dat, hoewel modulatie voor kracht werd beïnvloedt door grip vorm in sommige eenheden, slechts een kleine fractie van de variantie van de neurale populatie van elk hersengebied een gemixte selectiviteit voor grip vorm en kracht had. Informatie over grijpkracht kon daarom onafhankelijk van grip vorm worden geëxtraheerd.

Deze bevindingen suggereren een belangrijke rol voor AIP en F5 in het besturen van grijpkracht, samen met M1. F5 is waarschijnlijk ook betrokken met het plannen van grijpkracht, terwijl AIP en M1 waarschijnlijk een kleinere rol hebben in dit proces. Tenslotte, omdat informatie over grip vorm en grijpkracht onafhankelijk konden worden geëxtraheerd, laten deze resultaten zien dat grijpkracht vermoedelijk onafhankelijk van hand vorm is gecodeerd in het grijpnetwerk van de hersenschors. 


\section{Introduction}

This chapter introduces the importance of the hand, how it originated and how it shaped the evolution of the brain. I describe the most important cortical brain areas for hand control and what aspects of grasping movements they encode. I then explain what knowledge about grasping is still lacking and what the motivation for this study was.

I describe:

- How the hand originated and how both hand and brain evolved in primates (chapter 1.1.)

- How vision is used to guide grasping movements (chapter 1.2.)

- Which cortical areas are involved in controlling grasping movements (chapter 1.3.)

- How these cortical areas code force (chapter 1.4.)

- The motivation for this study (chapter 1.5)

- An overview of the experiment (chapter 1.6) 


\subsection{The evolution of hand and brain}

\subsubsection{Origin of the hand}

One of the foremost characteristics of our species, Homo sapiens, is that we stand upright and use our hands to manipulate complex objects. It is not just due to our great intellect and language that civilizations eventually emerged, our hands were just as essential. Our brains are greatly devoted to the control of our hands and this allows us to shape our hands in many different ways and carefully control the force we apply on objects. But how did this evolve?

Many mammals, such as rodents and cats, use their front paws for grasping food, but they cannot hold firmly to objects without using nails or both paws simultaneously. Grasping became more advanced about 60 million years ago, when a group of mammals, closely related to rodents, started living most of their lives in trees. Their front paws evolved longer fingers with skin ridges and a mobile thumb that allowed them to grasp tree branches much more effectively (Rose, 1994).

These mammals were the ancestors of the mammalian order Primates to which lemurs, tarsiers, monkeys and apes, including humans, belong. All primates have hands that are suitable for grasping, but only monkeys and apes have a fully opposable thumb, meaning that the thumb can touch the tips of all four fingers on the same hand, resulting in a much wider range of grip types. Having a variety of grip types allows these animals to effectively grab a great variety of objects and to carefully control how much force is applied on the 
object. This is not just useful in arboreal environments. Monkeys and apes can quickly grab food to eat later in a save place, groom group members, build nests, and even use tools.

Dexterous hand movements became more advanced in the human lineage, which even gave the oldest known human species, Homo habilis (handy man), its name. It is unclear what the exact evolutionary pressure was, that made human hands much more dexterous than hands of other apes, but there is a general agreement that this is somehow linked to tool use (Marzke and Marzke, 2000; Young, 2003).

\subsubsection{The brain behind the hand}

Hand anatomy strongly affects the dexterity of the hand, but it is equally important to have a complex neural network that can control these dexterous and voluntary movements. The brains of mammals are likely one of the most complex neural networks that can be found in the animal kingdom, as they have on average the largest brains compared to their body size, about ten times larger than that of reptiles of similar body size (Northcutt, 2002).

A key feature of mammalian brains is the large neocortex, which allows the animals to better process incoming sensory information and to better control their movements. Different areas of the neocortex started specializing in different functions over the course of evolution, with movement controlling areas on the anterior side and sensory information processing areas on the posterior side. 
After the discovery in 1870 that electrical stimulation of the frontal lobe of mammals evoke muscle activations on the opposite side of the body, this technique has been extensively used to understand the organization of the neocortex (Fritsch and Hitzig, 1870). They found that lowest intensities were required to elicit movements in the contralateral precentral gyrus (Brodmann's area 4), which is now called the primary motor cortex (M1), but also the anterior region, which is now called the premotor cortex (PMC), evoked movements. In M1 and PMC of most modern mammals there exists a mosaic organization of which movements are evoked by electrical stimulation and this organization crudely represents the arrangement of different body parts (Kaas and Stepniewska, 2016).

Within primates, the neocortex became not only larger, but also more specialized. By electrically stimulating the cortex of monkeys and humans, researchers observed that a crude motor map could be found in the PMC and M1 of primates that represent muscle activations from different body parts. They also found that the hand and face, which are often involved in tasks that require great precision, have disproportionally large representations (Penfield and Rasmussen, 1950). A caudal portion of primate M1 contains neurons that project directly from the cortex to motor neurons in the spinal cord that innervate shoulder, elbow, and finger muscles, possibly to coordinate very precise movements (Rathelot and Strick, 2009). This area of M1 was only found in higher primates and was dubbed "new" M1.

The initial idea was that every muscle was simply represented in a specific location of the motor cortex, but more precise microstimulation experiments later proved this idea wrong, 
as the same muscle could be activated by stimulating different sites of the cortex, and retrograde transneural transport of rabies virus from single muscles showed a widespread representation of these muscles in the neocortex (Rathelot and Strick, 2006).

It was later proposed that the cortical organization represents a map of muscle synergies that generate different movement types (Rathelot and Strick, 2006; Kaas and Stepniewska, 2016). By electrically stimulating different parts of the PMC and M1 of galagos, owl monkeys, squirrel monkeys, and macaque monkeys, stereotypical movements can be evoked that some have suggested to resemble reaching, grasping, or defending actions (Graziano et al., 2002; 2005; Stepniewska et al., 2014). Even though movements evoked by high-current stimulations could possibly also be spasms that happen to look like natural behaviors, these results emphasize a cortical layout of specific muscle groups and that this is similar in multiple primate species. This organization of the PMC and M1 possibly increased the effectiveness of certain common movements. However, to optimize these movements for different situations, they should be modifiable based on incoming sensory information.

The close vicinity of M1 to the somatosensory cortex suggests that haptic and proprioceptive information is used to execute movements accurately. Indeed, inactivating the somatosensory cortex results in awkward movements (Brochier et al., 1999). However, for grasping movements, animals need to rely on auditory and visual information to locate an object at a distance. I will focus here on how visual information is integrated by the brain, as this is the primary modality used by diurnal primates. 


\subsection{Visual guidance of grasping}

\subsubsection{Visual information pathways}

Visual information travels from the eyes, through the lateral geniculate nucleus, to the occipital lobe in mammals. At every step, the visual information is being processed to extract behaviorally relevant information. Already in the retina, basic visual information is processed due to the organization of ganglion cells to increase contrast perception. But most complex processing of information in mammals occurs in the neocortex.

Several occipital cortical areas are specialized in processing visual information such as object orientation and color (Hubel and Wiesel, 1968). The first cortical area where visual information arrives is the primary visual cortex (V1). From here, neurons project to many different parts of the brain and create a hierarchy of visual processing (Felleman and Van Essen, 1991). Of the different visual pathways, two pathways are particularly well described, one that travels from V1, via visual areas V2 and V4 to the inferotemporal cortex (ventral stream) and one that travels via V3 and the middle temporal area (MT) to the posterior parietal cortex (dorsal stream), see Figure 1.

These two streams were originally identified by Ungerleider and Mishkin in the macaque monkey (Ungerleider and Mishkin, 1982), and they proposed that different aspects of visual stimuli are processed in these streams. The ventral stream was proposed to involve 
object recognition and the dorsal stream processes object location in visual space. Neural responses in visual areas support this view, since neurons in V4 to object form (Desimone and Schein, 1987), and some neurons in the inferotemporal cortex respond only to very specific types of complex stimuli like faces (Freiwald and Tsao, 2010). On the other hand, neurons in MT do not respond strongly to object shape, but respond strongly to objects moving in visual space (Dubner and Zeki, 1971; Newsome et al., 1988; Britten et al., 1992). Furthermore, when shape encoding responses of neurons in the lateral intraparietal cortex (LIP), a high-level dorsal area, were directly compared to those in the anterior inferotemporal cortex (AIT), a high-level ventral area, the latter group was significantly more shape selective (Lehky and Sereno, 2007).

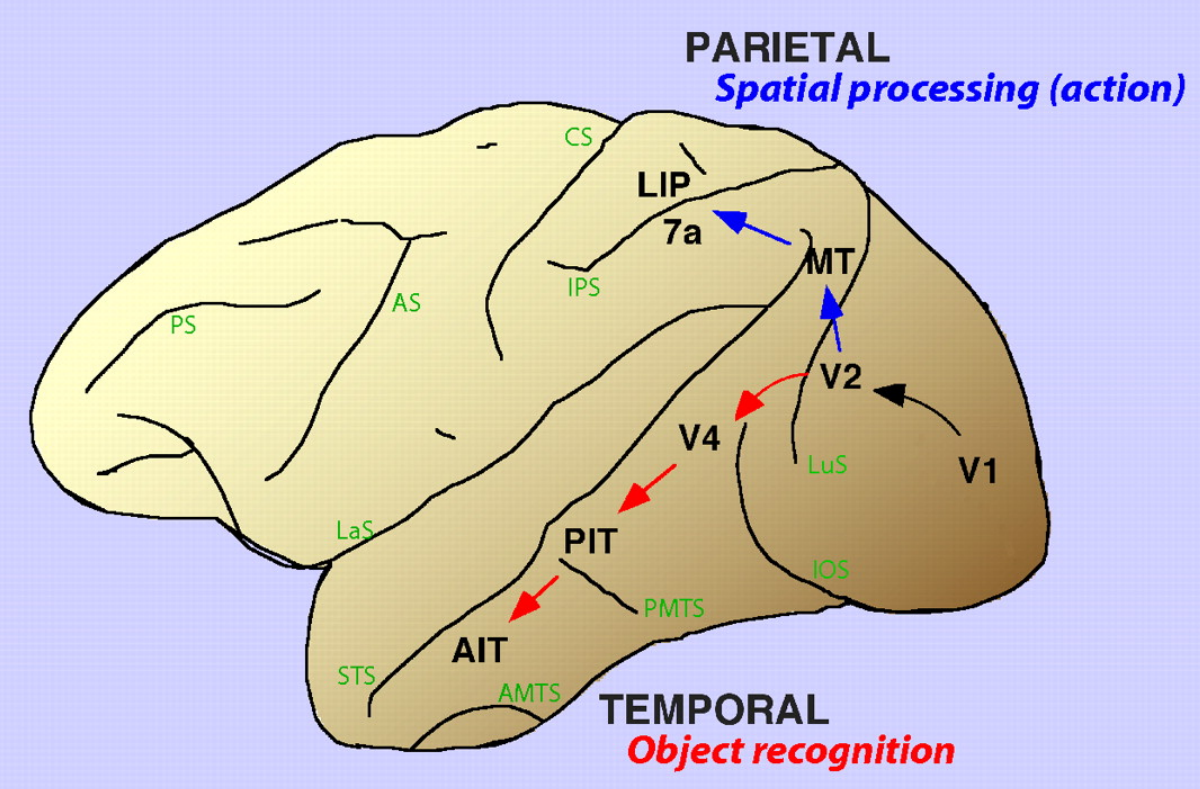

Figure 1. Schematic localization of visual pathways in the macaque monkey brain. 
(red arrows) to the temporal lobe, or along the dorsal pathway (blue arrows) to the parietal lobe. Labeled areas of the ventral pathway are: area V4, the posterior inferior temporal cortex (PIT), and the anterior inferotemporal cortex (AIT). Labeled areas of the dorsal pathway are: the middle temporal area (MT), the lateral intraparietal cortex (LIP), and area 7a. Labeled with green text are the sulci: principal sulcus (PS), arcuate sulcus (AS), central sulcus (CS), intraparietal sulcus (IPS), lateral sulcus (LaS), superior temporal sulcus (STS), anterior middle temporal sulcus (AMTS), posterior middle temporal sulcus (PMTS), inferior occipital sulcus (IOS), lunate sulcus (LuS). The label for LIP is placed next to the IPS, although LIP is actually located on the lateral bank of this sulcus and not visible from a surface view. Similarly, the label for MT is placed next to the STS, although MT is actually located in the posterior fundus of the STS and not visible from a surface view. (Figure adopted from Lehky and Sereno, 2007).

However, it was later proposed by Goodale and Milner (Goodale and Milner, 1992) that it is not so much the type of visual information processed in these two streams that defines them, but it is the way in which this information is used. Information in the ventral stream is used to form long-term perceptual representations to identify objects, while the dorsal stream information is used for actions such as saccades, reaching and grasping. How these two streams affect a subject's behavior becomes most apparent when only one of these streams is damaged.

In a study of Goodale and colleagues (Goodale et al., 1994), two patients were described, one patient (DF) had bilateral damage in the ventrolateral occipital region, and another patient (RV) had bilateral lesions of the occipitoparietal cortex. Both patients were exposed to a shape discrimination task and to a grasping task. As predicted by their hypothesis, patient DF had no problems with grasping and lifting various kinds of objects, but was 
unable to visually discriminate object differences. Conversely, patient RV had no difficulty with discriminating one object from another, but was unable to use visual information to place her fingers correctly on the circumference of irregularly shaped objects when asked to pick them up, a symptom known as 'visual apraxia'.

Activation patterns measured with functional magnetic resonance imaging (fMRI) are in line with these findings. One study compared fMRI activation patterns while human subjects observed intact or scrambled 2D object images and when subjects grasped or touched the object image (Culham et al., 2003). They found that a ventral stream area was significantly more active when viewing intact instead of scrambled objects, with no different activations when objects were grasped (grasping trials) or only touched (reaching trials). Dorsal stream areas located in the posterior parietal cortex (PPC) conversely showed a different activation pattern in grasping trials than in the reaching trials and did not respond differently to an intact or scrambled 2D object image. These lesion and fMRI results strongly suggest that ventral stream areas are involved in object recognition, while dorsal stream areas within the PPC are essential for using visual information to grasp objects.

\subsubsection{The posterior parietal cortex}

I have now described the integration of visual information in the PPC, and how this is relevant for performing accurate actions. In addition to visual information, this part of the cortex also receives strong input from somatosensory cortices (Cavada and Goldman-Rakic, 1989a). All this information is then integrated to be send to the motor cortices in the 
frontal lobe to influence upcoming movements (Cavada and Goldman-Rakic, 1989b). The motor cortices also send information like a copy of a motor plan, also called efference copy (Ford et al., 2012), back to the PPC to compare the planned movement to the executed movement for fine tuning.

In the brain of mammals closely related to primates, like rodents and tree shrews, the PPC is only a narrow strip of cortex between the somatosensory and visual cortex, extending ventrally to the auditory cortex (Kaas and Stepniewska, 2016), but in primates this area extended over the course of evolution to take up a larger proportion of the brain, as can been seen in Figure 1. Like in motor and somatosensory cortex, there is an overall somatotopy in the PPC, with the medial part representing the lower body and the lateral part representing the upper body in monkeys (Seelke et al., 2012) and humans (Huang et al., 2012). By applying long trains of electrical stimulation at specific areas of the PPC in galagos and new world monkeys (Stepniewska et al., 2014) stereotypical movements can be evoked that seem related to behaviors like feeding, defending, or grasping, and were almost identical to responses evoked by stimulating specific areas of M1 and PMC in these monkeys (Stepniewska et al., 2014) or in macaques (Graziano et al., 2002; 2005). What kind of movement was evoked depended very much on the exact location of stimulation in PPC, PMC, or M1, which was called the domain of that movement (e.g. grasping domain or reaching domain).

To emphasize the connection between the domains of different brain areas and to show the hierarchy of movement processes, Stepniewska and colleagues investigated the responses 
to electrical stimulation after inactivating specific domains (Stepniewska et al., 2014). Inactivating a specific domain in M1 greatly reduced or abolished movements evoked by stimulating the matching domain in PMC or PPC, while movements of nonmatching domains were mostly unaffected. Conversely, inactivating a domain in PMC or PPC, did not affect movements evoked from stimulating the matching domain in M1. However, the inactivation of PMC did suppress or alter movements evoked by stimulating the matching domain in PPC. These results strongly suggest a hierarchy for specific movements, with M1 being closest to movement execution, followed first by PMC and then by PPC (Kaas and Stepniewska, 2016).

Besides the integration of sensory information and the coordination of movements, it is also believed that the PPC is involved in making decisions about which actions to make. Originally it was believed that motor plans were generated after the decision was taken to perform a particular action, but it is now suggested that motor control and decision making are part of the same process (Cisek, 2012; Wolpert and Landy, 2012). Instead of only planning upcoming movements, it has been shown that areas in the PPC plan several movement plans in parallel (Klaes et al., 2011), integrate information about the reward that is linked to a particular action (Sugrue et al., 2004), and code information about which action is selected (Platt and Glimcher, 1999; Scherberger and Andersen, 2007; Pesaran et al., 2008; Cui and Andersen, 2011; for review, see McCoy and Platt, 2005; Andersen and Cui, 2009). Furthermore, it is even possible to influence the decisions of monkeys by electrically stimulating the parietal area LIP (Hanks et al., 2011). Many of these findings were also observed in areas of the PMC that have strong connections to parietal areas (Cisek and 
Kalaska, 2005; Pardo-Vazquez et al., 2008; Pesaran et al., 2008; Kennerley et al., 2009; So and Stuphorn, 2010; Pardo-Vazquez et al., 2011). This emphasizes the complex role of the PPC and PMC in the control of movement.

For the purpose of this thesis, I will now describe the area of the PPC particularly devoted to grasping movements. This will be followed by descriptions of the areas in the PMC and M1 that are also part of the cortical grasping network.

\subsection{Cortical grasping network}

\subsubsection{The anterior intraparietal area}

Observations of rhesus monkeys with lesions in the PPC revealed that they had an impaired grip formation and hand orientation (Haaxma and Kuypers, 1975). This suggested the existence of a grasp related area in the PPC, which was later identified in macaque monkeys by Taira and colleagues (Taira et al., 1990). They found a class of neurons in the posterior bank of the intraparietal sulcus (Figure 2D) specifically related to hand movements. This area was later called the anterior intraparietal area (AIP).

Many studies have later confirmed the findings that AIP neurons are strongly modulated by grip type and have extended this knowledge by finding that these neurons modulate their activity already while planning the movement (Baumann et al., 2009; Lehmann and Scherberger, 2013), and that these modulations can be used to predict upcoming grasping 
movements (Townsend et al., 2011; Menz et al., 2015; Schaffelhofer et al., 2015).

Furthermore, reaction time in a reach and grasp task could be predicted from AIP activity (Michaels et al., 2015).

The functional role of this area is probably best shown by studying the effect of inactivating this area. Gallese and colleagues found that after inactivating area AIP with the GABAagonist muscimol, monkeys had trouble preshaping the hand to fit to an object (Gallese et al., 1994). The monkeys could still grasp the object successfully by exploring the object with touch, but were unable to use visual information to preshape the hand.

Many other studies confirmed the high importance of visual information for AIP neurons (for review, see (Rizzolatti and Luppino, 2001; Janssen and Scherberger, 2015). Already in the study of Taira and colleagues it was described that the majority of hand-movement related neurons showed greater changes in activity when the monkeys grasped in a lighted room than in a dark room (Taira et al., 1990). Most importantly, many of these neurons showed a selective activation based on the configuration and orientation of the object to be manipulated, which was also confirmed in later studies (Sakata et al., 1995; Baumann et al., 2009; Chen et al., 2009; Schaffelhofer et al., 2015). Additional visual responses have been found in AIP as well, such as a selectivity for the location of a target in visual space (Lehmann and Scherberger, 2013; 2015) and a for binocular disparity (Srivastava et al., 2009). All these findings have given strong support to the idea of the important role of AIP in visually guiding grasping movements. 
Human homologues of AIP have also been identified. Binkofski and colleagues described that patients with lesions in the anterior bank of the intraparietal sulcus have selective deficits in the coordination of finger movements, while reaching is much less disturbed (Binkofski et al., 1998). Furthermore, patients with parietal lesions but intact AIP, showed normal grasping movements. This human AIP also showed specific fMRI activation when subjects were asked to make grasping movements in the MRI scanner (Binkofski et al., 1998; Culham et al., 2003), or when a subject imagined grasping movements (Aflalo et al., 2015).

As described in the previous chapter, areas in the PPC are believed to be higher order processing areas that receive sensory information and communicate with frontal areas to execute accurate and effective movements. Area AIP fits well within this view. I described in this chapter how it integrates many different types of grasp-relevant visual information and from anatomical evidence we know that it has many connections to the premotor cortex (Luppino et al., 1999). 

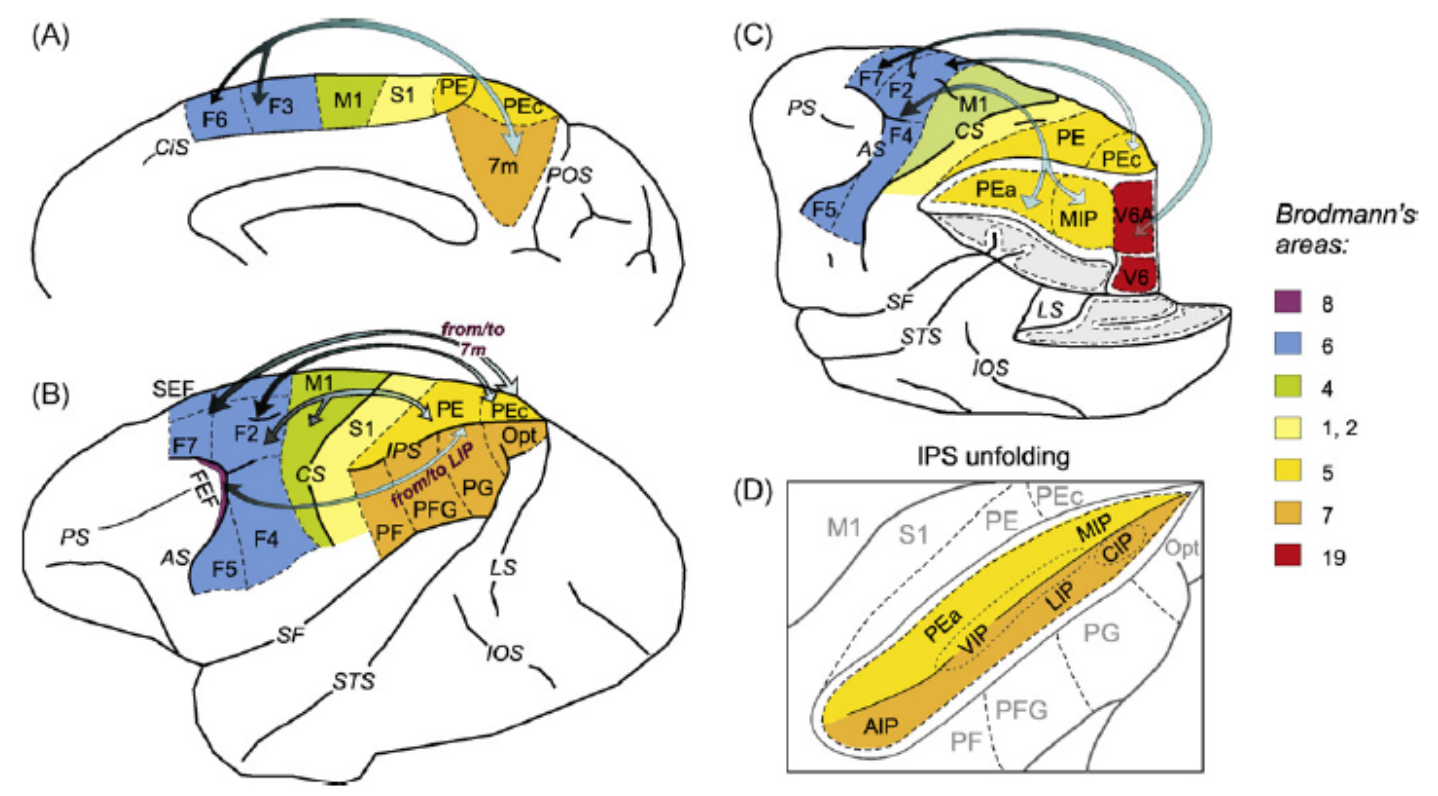

Figure 2. Macaque fronto-parietal network.

Different views of the monkey's brain with the main cortico-cortical connections linking different parietal and frontal areas. The color code distinguishes different areas according to the cytoarchitectonic parcellation of Brodmann. (A) Mesial view of the right hemisphere. (B) Lateral view of the left hemisphere. In (C) part of the parietal and occipital lobe have been "removed" to show the areas located in the medial bank of the intraparietal sulcus (IPS) and in the rostral bank of the parieto-occipital sulcus (POS). (D) Opening of the IPS to show the location of the areas buried in its medial and lateral banks. PS, AS, CS, SF, STS, LS, IOS indicate principal, arcuate, central, Sylvian, superior temporal, lateral, inferior-occipital sulci. (Figure and legend adopted from Ferraina et al., 2009).

\subsubsection{Area F5}

One of the foremost areas to which AIP projects, is the anterior portion of the ventral premotor cortex, called F5 (Luppino et al., 1999), see Figure 2B. Even before properties of AIP neurons were described, Rizzolatti and colleagues showed that neurons from F5 discharge in relation to actions like reaching, grasping, and holding (Rizzolatti et al., 1988). 
And, like AIP, inactivation of this area with muscimol severely impaired the ability to preshape the hand before grasping an object (Fogassi et al., 2001).

F5 neurons respond altogether very similar to those in AIP, they are also modulated by grip type and visual properties of objects, both during movement and during planning (Rizzolatti et al., 1988; Rizzolatti and Luppino, 2001; Fluet et al., 2010; Lehmann and Scherberger, 2013; Janssen and Scherberger, 2015; Lehmann and Scherberger, 2015; Menz et al., 2015; Schaffelhofer et al., 2015; Vargas-Irwin et al., 2015), but the movement related responses, like grip type, are on average stronger in F5, whereas visual stimuli, like object configurations, are stronger in AIP. Also reaction time for grasping movements was predicted better from F5 than from AIP (Michaels et al., 2015).

The stronger relation to movement execution of F5 compared to AIP is also evident in its anatomical connections to other areas. Unlike AIP, F5 projects to the hand field of M1, the brainstem and spinal cord (Borra et al., 2010). It is therefore possible to directly influence hand muscles, although it is believed that its main effect on hand movements is via M1, on which is has a strong facilitation effect (Shimazu et al., 2004).

\subsubsection{The hand area of M1}

As mentioned before, the primary motor cortex is believed to be the cortical area closest to movement execution, and the hand represents a relatively large portion of it. Many neurons of the hand area of M1 project directly to the spinal motoneuron pools (Muir and Lemon, 1983) and are part of the corticospinal tract, also called pyramidal tract, named for the 
medullary pyramids formed by the fibers of this projection. Some neurons in the caudal portion of M1, which have been called "new" M1 (Rathelot and Strick, 2009), even make direct monosynaptic connections with motoneurons (Cheney and Fetz, 1980; Bennett and Lemon, 1994; Rathelot and Strick, 2006) and are called "cortico-motoneuronal cells". Lesions of the pyramidal tract results in a loss of speed and agility in several movements and in a loss of fractionation which is especially notable in hand movements, due to the disability to move fingers individually (Lawrence and Kuypers, 1968).

A severe effect on hand movements can also be observed after inactivating contralateral M1 (Kubota, 1996; Schieber and Poliakov, 1998; Brochier et al., 1999), which is much stronger than the effect of deactivating AIP (Gallese et al., 1994) or F5 (Fogassi et al., 2001). Not surprisingly, many neurons in M1 are selective for grip type (Muir and Lemon, 1983; Mollazadeh et al., 2011) and it is possible to predict hand movements from M1 neural activity more accurately than from AIP and F5 (Carmena et al., 2003; Menz et al., 2015; Schaffelhofer et al., 2015). However, AIP and F5 activity before movement was a better predictor of upcoming movements than M1 activity before movement, indicating a relatively smaller role of M1 in movement planning.

I have described the cortical pathway from visual information to executing hand movements. Three cortical areas are particularly involved in controlling hand shape: AIP, F5, and M1. However, another essential parameter to successfully grasp objects is the amount of force applied on the object. We will now describe the role of areas AIP, F5, and M1 in the control of force. 


\subsection{Force coding in the cortical grasping network}

\subsubsection{Force coding in M1}

Because they project directly to spinal motoneurons, pyramidal tract neurons of M1 were among the first to be studied to investigate the relation between exerted force and neural firing rate. Already in the 1960s, Edward Evarts recorded the activity of these neurons while macaque monkeys made flexion and extension movements with the wrist under different load conditions (Evarts, 1968). He found that the discharge rate of most recorded pyramidal tract neurons was related primarily to force and only secondarily to the direction and amplitude of displacement. This was also the case when only corticomotoneuronal cells were investigated (Cheney and Fetz, 1980).

Many other types of force coding in M1 have later been documented in different experimental paradigms, such as reach tasks (Georgopoulos et al., 1992; Taira et al., 1996; Boline and Ashe, 2005; Gupta and Ashe, 2009) or tongue-protrusion tasks (Arce-McShane et al., 2014). However, the focus here will be on studies that investigated the neural coding of grip force.

Among the first researchers to study the relation of activity of M1 neurons to grip force, were Smith, Hepp-Reymond and Wyss (Smith et al., 1975). They trained macaque monkeys to compress a strain gauge between the thumb and forefinger, a so-called precision grip, at 
different instructed force levels from 1 to $2.5 \mathrm{~N}$. Spiking activity of M1 neurons was recorded simultaneously and they found several neurons of which firing rate correlated linearly with exerted force or with the rate of force change (i.e. the first derivative of force). They also mentioned that the change in discharge frequency coincided or occurred after the onset of EMG activity.

Hepp-Reymond and colleagues continued investigating the cortical control of grip force in a series of studies. Some notable findings were that the earlier described linear correlation between static grip force and neural firing rate is strongest in the small range of 0.1 to $1 \mathrm{~N}$ (Hepp-Reymond et al., 1978) and that tonic discharges during the hold period were most common (Hepp-Reymond and Diener, 1983). They also demonstrated similar effects when only cortico-motoneuronal cells were considered (Maier et al., 1993). Furthermore, they described that force coding depends heavily on the context. When monkeys performed a task with three force levels, the change in firing rate between the first two steps was significantly smaller than in a task that consisted only of those two force steps (HeppReymond et al., 1999).

A functional relationship between M1 and grip force was observed when M1 was inactivated by a muscimol injection. Similar to earlier M1 inactivation experiments (Lawrence and Kuypers, 1968; Kubota, 1996; Schieber and Poliakov, 1998) Brochier and colleagues observed that M1 injections produced a deficit in the ability to perform individual finger movements (Brochier et al., 1999), but they also noticed a reduction in peak grip forces applied to an object, shortly before the monkeys lost the ability to perform 
the task. Importantly, this was not observed after a muscimol injection in the somatosensory cortex, which conversely resulted in an increase in peak grip forces, likely due to a reduction in cutaneous feedback.

Finally, M1 signals have been used to successfully predict grip force (Carmena et al., 2003; Flint et al., 2014; Milekovic et al., 2015; Bataineh et al., 2016), providing further evidence that these signals are well represented in this brain area.

\subsubsection{Force coding in F5}

Because of its strong representation of hand movements and connections to M1, the brainstem and spinal cord (Borra et al., 2010), F5 is another obvious target to search for grip force coding.

In addition to her work on grip force coding in M1, Hepp-Reymond also investigated grip force coding properties of neurons in two regions of the ventral premotor cortex (PMv) (Hepp-Reymond et al., 1994). Like in M1, she found neurons that had a significant correlation between exerted force and neural firing rate. However, she did describe that the percentage of force coding neurons was lower in the premotor cortex than in M1. When two regions of PMv, a caudal and a rostral region, were compared, the rostral region, which roughly corresponds to $\mathrm{F} 5$, showed the highest incidence of force coding neurons and overall more similarity in responses to the hand field of M1. Another similarity to M1 was shown in one of her other studies, namely that force coding in the premotor cortex is also context-dependent (Hepp-Reymond et al., 1999). 
Later studies have not always been successful in finding force coding neurons in premotor cortex. Boudreau and colleagues did not find a correlation between the activity of PMv neurons and exerted grip force, but also mentioned this may have been because the monkeys hardly varied grip force for different object loads (Boudreau et al., 2001). Interestingly, grip force did vary for different object textures and an effect of texture on firing rate was observed, which possibly could have been related to grip force.

Other studies have looked at the dorsal premotor cortex for grip force coding, but consistently found low percentages of neurons coding grip force (Hepp-Reymond et al., 1999; Hendrix et al., 2009) and lower decoding performances than M1 (Carmena et al., 2003; Bataineh et al., 2016). Conversely, when grip force decoding was investigated for PMv, a preliminary report found predicting performances just as high as M1 (Bataineh et al., 2016).

All in all, it can be said that grip force is generally quite poorly represented in premotor cortex, but PMv, and especially its rostral portion F5, seems to be an exception to this, as it is much closer to M1 in respect to grip force.

\subsubsection{Force coding in AIP}

When force-coding neurons are not as common in the premotor cortex as in M1, they are likely even less common in the parietal cortex, as this area is even less directly involved in controlling movements. 
Classical work from Kalaska and colleagues supports this view. They showed that neurons in the superior parietal lobule, or area 5 (Figure 2), respond to movement kinematics in a reaching task, but are only weakly affected by directional loads, contrary to M1 neurons (Kalaska et al., 1990). And a later study showed that area 5 neurons were more strongly active or exclusively active in a reaching task than in a isometric task (Hamel-Pâquet et al., 2006). The conclusion from these findings was that area 5 neurons reflect movement kinematics, but not kinetics.

However, the inferior parietal lobule, or area 7 (Figure 2), was not addressed in this study. Based on the finding that parietal patients suffer from defective control of the direction of reach force (Ferrari-Toniolo et al., 2014), the responses of neurons in areas PF and PFG of the macaque inferior parietal lobule to directional force were investigated. The researchers found that a high percentage of these neurons responded to isometric force output and that the population vector predicted the force output better than cursor position (FerrariToniolo et al., 2015).

Since area AIP borders PF (Figure 2), it is likely that a population of neurons coding force, most likely grip force, can also be found here. Data obtained from fMRI experiments point in this direction. When subjects were required to produce grip force with the right hand on an object, several motor, premotor, but also parietal areas showed an increased activation in the left or both hemispheres (Ehrsson et al., 2003; Keisker et al., 2010; Neely et al., 2013). Even when subjects were instructed to only imagine right-handed grasping with a 
certain amount of force, an increased activity in bilateral premotor and inferior parietal cortex was observed, and this scaled with the amount of imagined force in the right hemisphere (Mizuguchi et al., 2014). The right/ipsilateral intraparietal cortex also showed higher activations when subjects were required to anticipate and coordinate grip force when lifting an object (Ehrsson et al., 2003), and in tasks when subjects were required to statically hold a fixed force level instead of dynamically squeezing an object (Keisker et al., 2010; Neely et al., 2013). Even though fMRI activation areas are rather large, it is quite likely that the human homologue of AIP is activated by these grip force tasks as well. This would then also suggest that neurons responding the grip force could be found in this area.

Probably the strongest evidence for the involvement of AIP in the control of grip force comes from an experiment that used transcranial magnetic stimulation (TMS) to induce a virtual lesion of AIP (Davare et al., 2007). They found that after inactivating left-sided AIP in right-handed individuals, grip force scaling was selectively impaired for both hands. Interestingly, inactivating right-sided AIP had no effect on grip force and to induce an effect on hand shaping, AIP had to be inactivated bilaterally. Furthermore, they describe that AIP needed to be inactivated $270-220 \mathrm{~ms}$ before object touch to impair hand shaping and 170120 ms before object touch to impair grip force scaling. These findings suggest an important role of AIP in the control of grip force and that this can be temporally dissociated from the effect on hand shaping. 


\subsection{Motivation for this study}

I have now described how the hand emerged over the course of primate evolution and how the brain evolved to control it with great dexterity. Three cortical areas are essential for hand control: the hand area of the primary motor cortex (M1), the hand area of the ventral premotor cortex (F5), and the anterior intraparietal area (AIP). Much is already known about how these areas are involved in shaping the hand. However, much less is known about how these areas are involved in controlling grip force, another essential parameter for successful grasping movements. This study aims to increase our knowledge about the cortical control of grasp force.

It has been known for a long time that M1 and, to a lesser extent, F5 neurons code grip force (Smith et al., 1975; Hepp-Reymond et al., 1994). However, to our knowledge, no study has shown these properties for AIP neurons. This is not a trivial knowledge gap, as we know that AIP is an essential region for the neural control of grasping (for review, see (Rizzolatti and Luppino, 2001; Janssen and Scherberger, 2015). Whole-brain scanning techniques have consistently found an increased activation of AIP and surrounding areas during grip force control (Ehrsson et al., 2003; Keisker et al., 2010; Neely et al., 2013) and inactivating AIP with TMS resulted in an impairment to control grip force (Davare et al., 2007). The first question we will address in this study is therefore: How is grasp force coded at the single neuron level in AIP? 
It has also not been investigated in great detail how grasp force planning is represented in neural signals. While delayed grasping paradigms have frequently been used to disentangle the influence of grip type planning from active grasping movements on neural activity (Taira et al., 1990; Sakata et al., 1995; Baumann et al., 2009; Fluet et al., 2010; Vargas-Irwin et al., 2010; Townsend et al., 2011; Fattori et al., 2012; Lehmann and Scherberger, 2013; 2015; Michaels et al., 2015; Schaffelhofer et al., 2015), only few have used this paradigm to investigate grasp force planning (Zaepffel et al., 2013; Milekovic et al., 2015). These studies found an effect of a grip force cue on EEG activity (Zaepffel et al., 2013) and on M1 LFP activity (Milekovic et al., 2015), but only from LFP activity the force level could be predicted and only with minor accuracy compared to grip type prediction. The effect of grasp force planning on spiking activity remains unclear.

One explanation for the much lower predictability of grasp force is that it relies mostly on haptic feedback and is therefore not planned very well in advance. However, an experiment that tested force control with a myoelectric hand prosthesis found that human subjects relied more on information about the prosthesis velocity than on force feedback, indicating a high importance of planning grasp force before touching an object (Ninu et al., 2014). The second question addressed in this study is therefore: How is grasp force planning represented in the neural spiking activity of neurons in AIP, F5, and M1?

Even though we know that grip type has a major effect on the neural signal in areas like AIP, F5, and M1 (Rizzolatti and Luppino, 2001; Janssen and Scherberger, 2015), most studies looking at grip force representation only did this for one grip type, usually the 
precision grip (Hepp-Reymond et al., 1978; Hepp-Reymond and Diener, 1983; Maier et al., 1993; Hepp-Reymond et al., 1994; 1999; Ehrsson et al., 2003; Davare et al., 2007; Neely et al., 2013). An interesting question is whether grip type and force coding are dependent on each other, just like muscle activations, or if they are coded independently in the brain.

Two studies speak in favor for the latter hypothesis. First, Davare and colleagues found that hand shaping and grip force control are distorted by AIP inactivation at different time points (Davare et al., 2007). Second, Hendrix and colleagues investigated the influence of grasp force on the neural activity of M1 and dorsal PMC for a number of different object shapes and found no interaction between grasp force and grasp dimension (Hendrix et al., 2009). However, much is still unknown about how grip type and force coding interact, which leads to our third question: What is the relation between grip type and force coding?

\subsection{Overview}

To answer these three questions, we trained two macaque monkeys to perform a delayed grasping task with two grip types and three force levels. While the monkeys performed the task, we recorded the neural signals in AIP, F5, and M1 with floating microelectrode arrays. In chapter 2 we give a detailed overview of the methods used to answer these questions. In chapter 3 we show the results obtained in this study with several different analyses. Finally, in chapter 4 we will summarize the results and discuss the meaning of these findings in the current literature. 
The terms 'grip force' and 'grasp force' are used interchangeably when I refer to the literature in this thesis. However, I use the term 'grasp force' when I refer to our task design to emphasize the reach and grasp action. 


\section{Methods}

This chapter describes the methodological procedures that were undertaken to answer the research questions presented in the last chapter.

Here I describe:

- The basic procedures of the animal training and the experimental setup (Chapter 2.1.)

- The task performed by the monkeys (Chapter 2.2.)

- The design of the force sensing handle (Chapter 2.3.)

- The surgery and implantation of the micro-electrode arrays (Chapter 2.4.)

- The neural data recording and spike sorting (Chapter 2.5.)

- The behavioral, analog, and neural data analysis (Chapter 2.6.)

Natalie Nazarenus and Lukas Schad assisted in animal training and recording. Matthias

Dörge assisted in the design and construction of the force sensing handle. Hansjörg

Scherberger performed the surgery and implantation. Benjamin Dann and Jonathan Michaels assisted in the neural data analysis. 


\subsection{Basic procedures}

One male (Monkey B) and one female (Monkey S) rhesus macaque (Macaca mulatta, 13.5 and $10.1 \mathrm{~kg}$, respectively) were used in this study. The monkeys were purpose bred and held in an enriched environment with one or two conspecific companions. Before experiments started, the monkeys were habituated to move from their cages into a custommade primate chair at their own speed. Great care was taken to avoid any actions that might evoke any unnecessary stress in the animals. Facial expressions and behavior were carefully monitored for signs of stress. All animal care and experiments with the animals were performed in accordance with German and European law and in agreement with the Guidelines for the Care and Use of Mammals in Neuroscience and Behavioral Research (National Research Council (US), 2003) as well as with the NC3Rs Guidelines (Watson, 2007).

Both monkeys were habituated to comfortably sit in a primate chair with the head fixated. Before starting the task paradigm, the monkeys were placed in front of a grasping handle at a horizontal distance of $\sim 26 \mathrm{~cm}$ in a dark, electrically shielded room. One or two capacitive sensors (Model EC3016NPAPL; Carlo Gavazzi) located at the level of the monkey's midtorso were placed in front of monkey B and S, respectively, and served as handrest buttons. Monkey S performed the task with her right hand and was required to keep her left hand on the handrest button for the entire trial duration. Monkey B performed the task with his left hand and his right arm was placed in a long tube, preventing it from interacting with the handle. Visual cues were projected from a TFT screen (CTF846-A; Screen size: 8" digital; Resolution 800x600; Refresh rate: $75 \mathrm{~Hz}$ ) onto the center of the handle via a half 
mirror. The TFT screen was masked so that direct view of the image was not possible. Eye movements were tracked with an infrared optical eye tracker that was calibrated at the start of each session. Eye tracking and the behavioral task were controlled with customwritten software implemented in LabView Realtime (National Instruments) with a time resolution of $1 \mathrm{~ms}$. Monkey behavior was continuously monitored via an infrared camera while the monkeys performed the task.

\subsection{Task paradigm}

The monkeys were instructed with visual cues how and when to grasp (Figure 3A). A trial was initiated by placing the acting hand on the handrest button and it had to remain here until the Go signal was given. A red dot was projected in the same location as the grasping handle and functioned as eye fixation target. The monkeys were required to fixate this dot for the entire trial duration. After fixating the dot for the first 400-500 ms (Fixation epoch), two spotlights illuminated the grasping handle for $800 \mathrm{~ms}$ and an instruction cue was visible on the left or right side of the fixation dot during this time (Cue epoch). The location of this cue instructed which grip type had to be made. A cue on the left side instructed a whole-hand grip (opposition of fingers and palm), while a cue on the right side instructed a precision grip (opposition of index fingers and thumb). As can be seen in Figure 3A, the cue consisted of a green disk and a grey bar in front. Within this grey bar, the height of a white square indicated how much force the monkey had to apply when executing the grasp. When the square was at the bottom of the bar, 0-5 $\mathrm{N}$ of force was required (Low Force), when this was one level higher, 5-9 $\mathrm{N}$ of force was required (Medium Force), and when the white square was in the middle of the bar, 9-12 $\mathrm{N}$ of force was required (High Force). The 
same force values were used for Whole-hand- and Precision grip trials. The selected force range was based on the amount of force the monkeys naturally applied to the handle before being trained on the force cues. After the cue epoch, illumination and instruction cues were turned off and the monkeys were required to memorize the instruction and continue fixating for 500-700 ms (Memory epoch). At the end of the Memory epoch, the fixation dot would briefly blink to indicate that movement could now be initiated (Go signal). The monkey would now reach for the handle and the moment the hand left the handrest button was termed Movement initiation. The Touch event was the moment the hand broke the infrared light barrier behind the handle, in case of a whole-hand grip, or when the thumb and index finger touched the electronic touch sensors on the side of the handle, in case of a precision grip. After Touch, the bar with the force cue would reappear and within it, a red slider bar was visible that moved up and down when force applied on the handle increased or decreased, respectively. The monkey had to bring the top of the red slider bar within the white square and keep it there for one second (Hold epoch). When this was done correctly, the monkey was rewarded with a small amount of water or fruit juice. The moment the valve of the reward system opened was called the Reward event. To signal when the valve was open, a high-pitched beep sound was played during this time. The handle was only visible during the cue epoch, hence the entire grasp and hold action was done in darkness. 


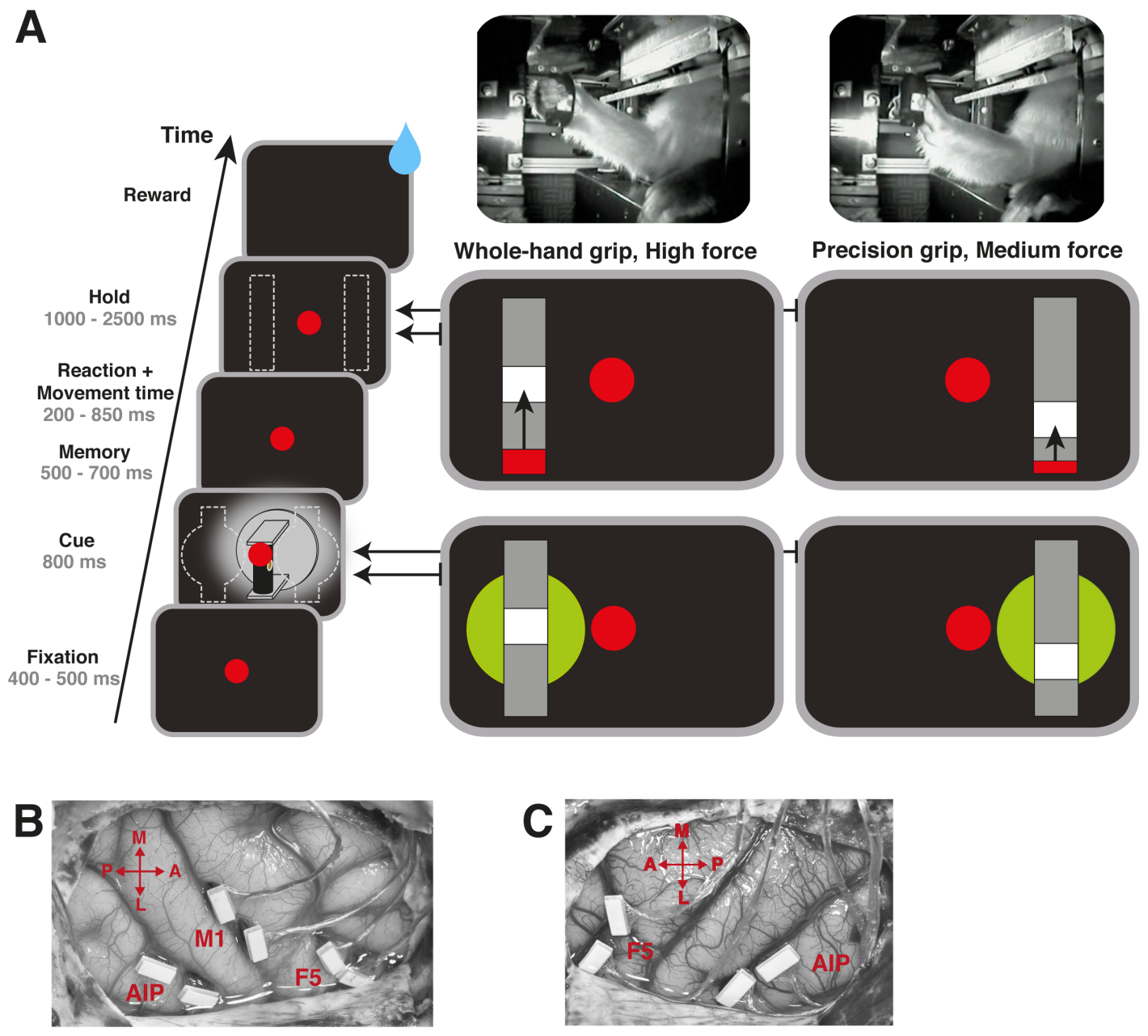

Figure 3. Task paradigm and recording locations.

A. Task paradigm. Each rectangle on the left shows the image visible during one particular epoch. The specific Cue and Hold epochs of two example conditions are shown on the right. Arrows in the Hold epoch indicate to where the red bar has to move in order to complete the task (arrows were not presented to the monkeys). The two pictures on the top show monkey S performing a whole-hand grip and a precision grip. B. Picture of arrays implanted in the right hemisphere of Monkey B. From left to right: arrays implanted in AIP, M1, and F5. C. Picture of arrays implanted in the left hemisphere of Monkey S. From left to right: arrays implanted in F5 and AIP. Orientation cross: P - Posterior, A - Anterior, L - Lateral, M - Medial. 
To investigate how well the information about the force conditions was used during the Cue epoch, we trained monkey B on a variation of the task were the white square did not reappear in the Hold epoch. This compelled the monkey to remember the force condition from the Cue epoch, as it was no longer able to simply bring the red slider bar to the white square to complete the trial.

To adequately measure grasp force, we wanted the monkeys to generate force by contracting the hand and not by strongly pushing or pulling on the handle. Monkey S was therefore not allowed to push or pull with more than $11.5 \mathrm{~N}$ in any condition and for monkey B this was 11.5, 23, and $34.5 \mathrm{~N}$ in the Low, Medium, and High Force condition, respectively. In this case, monkey B was less restrained than monkey S, because he had more difficulty with learning the Medium and High Force conditions. However, by the time monkey B had learned the task, he rarely pushed or pulled with more than $12 \mathrm{~N}$ in any of the conditions.

The task consisted of two grip type conditions and three force conditions, resulting in six different conditions: Whole-hand Low Force (WLF), Whole-hand Medium Force (WMF), Whole-hand High Force (WHF), Precision Low Force (PLF), Precision Medium Force (PMF), and Precision High Force (PHF). All trials were pseudo randomly interleaved. Trials were initially drawn from a pool of 30 trials, containing 5 copies of the six conditions. Successful trials were removed and the pool was refilled with a copy of each of the six conditions whenever the pool contained less than 25 trials (i.e. the pool size always varied between 25 to 30 trials). Unsuccessful trials stayed in the pool to force the monkey to complete each 
condition roughly the same amount of time. Hence, when one condition was not performed successfully as often as others, it would appear slightly more frequently, but the monkeys could never know with full certainty which condition would appear in the next trial.

\subsection{Force sensing handle}

In order to measure grip type and force simultaneously, we designed a new grasping handle. This handle was similar to the PVC handle used in previous experiments in our lab (Baumann et al., 2009; Fluet et al., 2010; Townsend et al., 2011; Lehmann and Scherberger, 2013; Michaels et al., 2015); an infrared (IR) light barrier was located behind the handle to detect a whole-hand grip and two touch sensors on the side of the handle could detect precision grips. However, unlike the PVC handle, this handle consisted of an air-filled rubber tube (figure 4a) connected to an air pressure sensor (figure 4b). Furthermore, because the previously used force-sensitive resistor sensors did not function on the rubber tube, electronic touch sensors replaced them. The air pressure in the tube was set to a value between 190 and $210 \mathrm{kPa}$ before an experiment started, and this typically did not change by more than $2 \mathrm{kPa}$ over the course of an experiment. The air-filled tube was surrounded by two convex metal plates, covered by another rubber tube, onto which the precision grip sensors where placed. Within the air-filled tube was a solid metal bar. This design was able to resist the rough actions of the monkey, prevented the tube from greatly changing shape during experiments, and the rubber prevented the precision grip sensors from short-circuiting. The precision grip sensors were regularly cleaned to not lose sensitivity. The handle was mounted on an S-shaped force sensor (KD24s, ME-Meßsysteme $\mathrm{GmbH}$ ) to additionally measure push and pull force. 

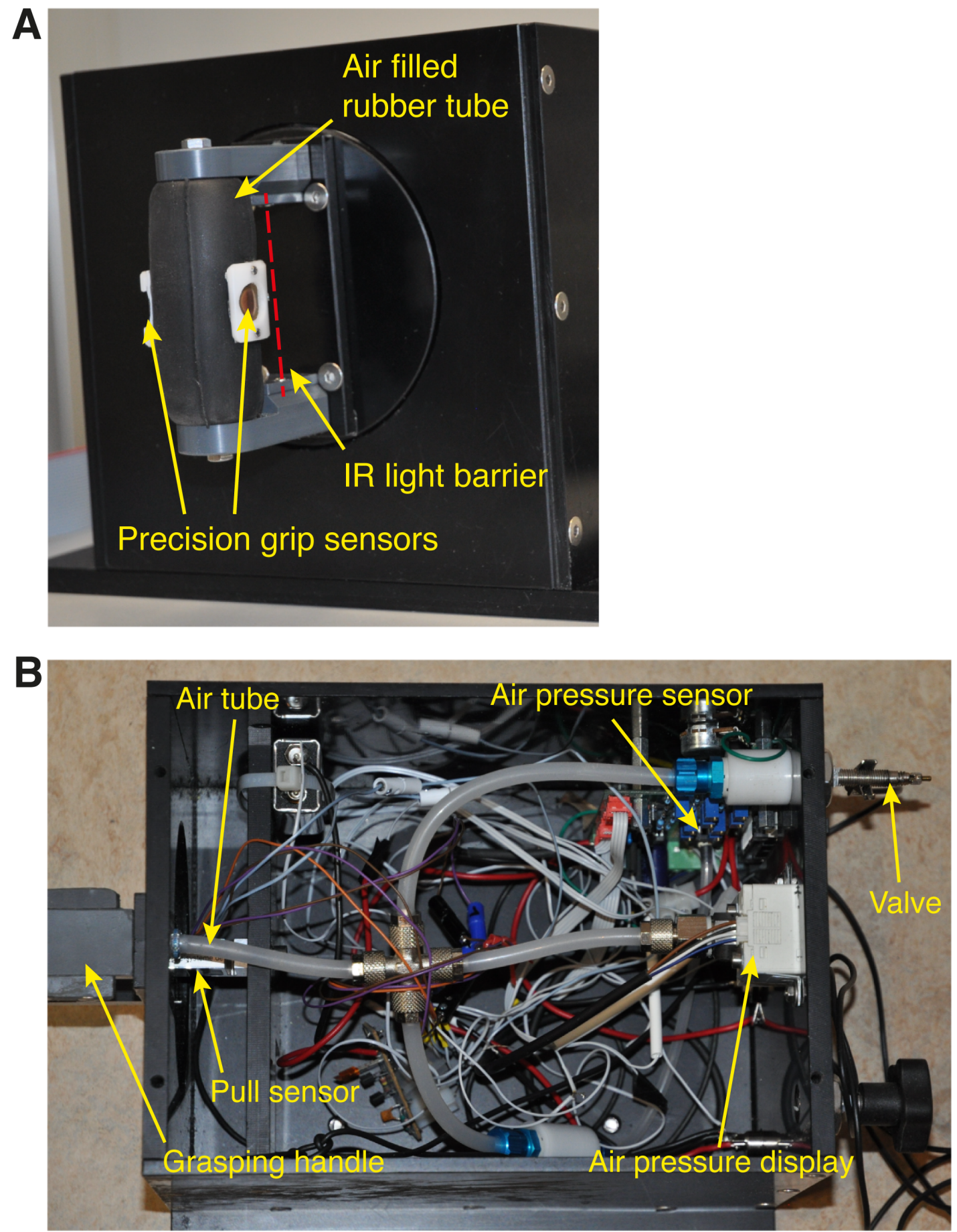

Figure 4. Force sensing handle.

A. Front view of the handle. Whole-hand grips could be detected when the IR light barrier was broken and precision grips could be detected when thumb and index finger simultaneously touched the sensors on both 
sides and a low current went through the hand. The amount of force applied on the handle was measured by measuring the change in air pressure in the rubber tube. B. Top view of the box behind the handle. The four plastic air tubes are connected to an air valve, an air pressure sensor, an air pressure display, and to the grasping handle. Air pressure was controlled by manually pumping air in the tubes, or by releasing air from the valve on the right side. The air pressure sensor located on the top-right measured small changes in air pressure in the tubes and send this to the recording system. The air pressure display is a separate, less accurate air pressure sensor with a display that was only used to roughly know the amount of air pressure in the tubes, to keep this similar from session to session. A pull sensor was located behind the grasping handle on the left side to record and control the amount of force the monkeys applied on the handle by pushing or pulling.

Changes in air pressure in the tube resulted in voltage changes and grip force was calculated as the difference $(\Delta$ Voltage) between voltage $(\mathrm{mV})$ recorded while the monkey touched the handle and voltage recorded in the fixation period just before the handle was touched. In order to estimate with how much force $(\mathrm{N})$ the handle was grasped, the change in voltage during experiments was compared to the voltage change when a fixed amount of force (ranging from 2-20 Newton) was applied simultaneously on two sides of the handle. Push force (N) was measured in the same way, except that the force was now applied only on the front side. Pull force (N) was simply negative push force.

Figure 5 shows recorded $\Delta$ Voltage after applying a fixed amount of force on the handle. We applied several linear and non-linear fits (Matlab function: $c f t o o l$ ) with intercept to zero on the data. It can be seen in figure 5 that a linear model (black line) gave a good fit for the grip force $\left(\mathrm{R}^{2}=0.96\right)$ and push force $\left(\mathrm{R}^{2}=0.93\right)$. The non-linear power model gave an even 
better fit for the grip force (magenta line, $\mathrm{R}^{2}=0.99$ ), but did not improve for the push force.

For calculating the grip force, we therefore used the following formula:

$$
y=4.3 x^{\wedge} 1.3
$$

And for calculating the push force, we used:

$$
y=-43.4 x
$$

Where $y$ is force $(\mathrm{N})$ and $x$ is $\Delta$ Voltage $(\mathrm{mV})$.
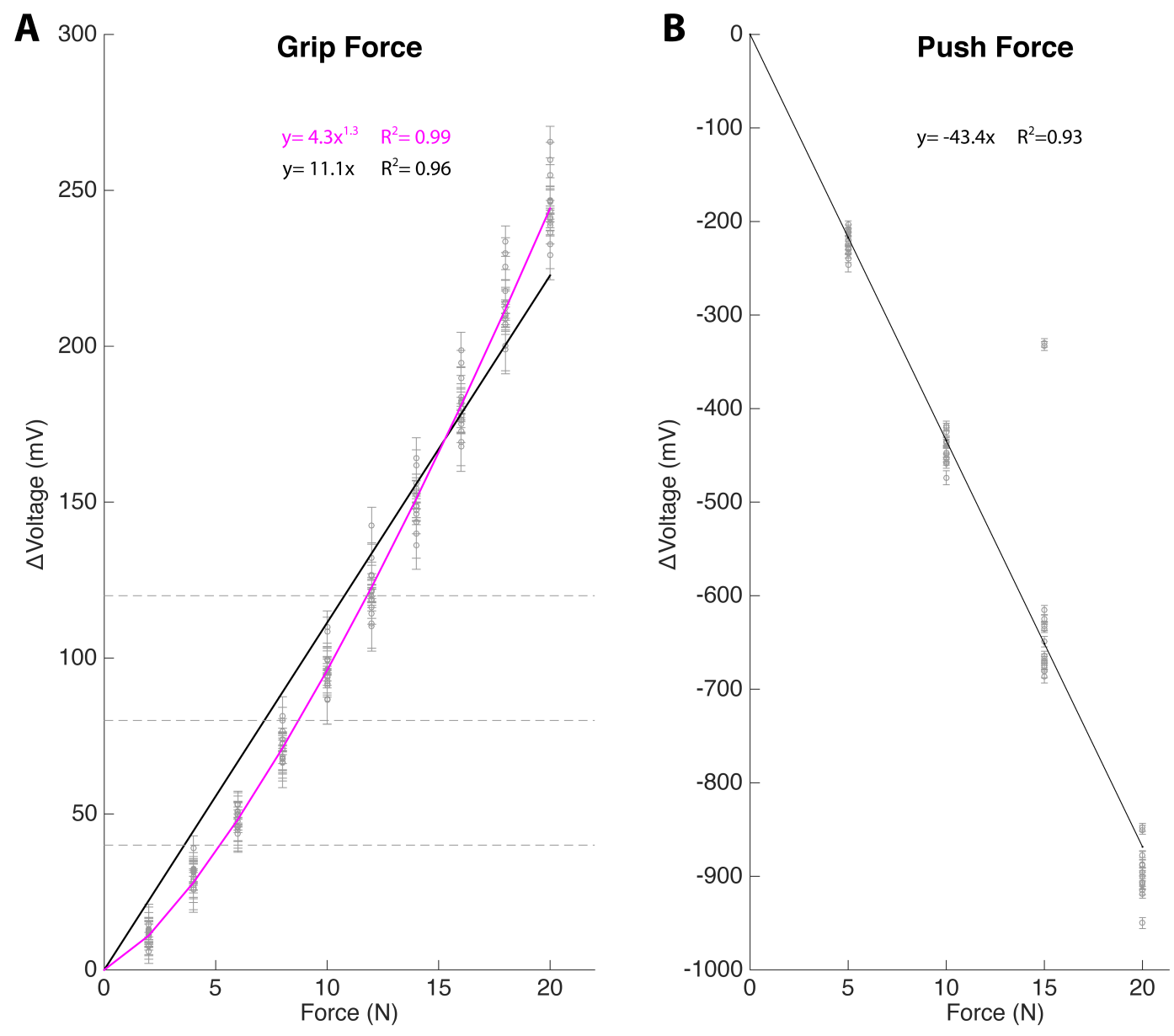

Figure 5. Force calibration.

The $\mathrm{x}$-axis shows the amount of force applied on the handle and the y-axis shows the change in voltage from baseline ( $\Delta$ Voltage) measured by the recording system. Every grey circle shows the mean value of the $\Delta$ Voltage of a recording sample over a few seconds, with standard deviation. The black line shows the linear 
fit and the magenta line the non-linear fit. The formulas on the top of the figure correspond to the line with the same color. A. Grip force calibration. Grey horizontal dashed lines show the upper and lower borders of the Low Force $(0-40 \Delta \mathrm{mv})$, Medium Force $(40-80 \Delta \mathrm{mv})$, and High Force $(80-120 \Delta \mathrm{mv})$ condition. B. Same as A, but for push force calibration.

This method gives a good approximation of the amount of force applied at the tip of the thumb and index finger during a precision grip and, due to the convex plates surrounding the tube, the overall force applied by the hand during a whole-hand grip. Note that the different force conditions were based on linearly increasing $\Delta$ Voltage $(40,80$ and $120 \mathrm{mV}$; dashed lines in figure 5). Because of the nonlinear relationship between $\Delta$ Voltage and force, the lower force conditions had a slightly wider range than the higher force conditions, but this was not considered to be relevant for the task design.

\subsection{Surgery and implantation}

Surgery and implantation procedures have been described before (Townsend et al., 2011; Michaels et al., 2015). Monkey S and monkey B were implanted with 2 floating microelectrode arrays (FMAs; MicroProbe for Life Science) in area F5 and 2 in AIP (Figure 5). Monkey B was also implanted with 2 additional FMAs in area M1 (Figure 5B). All arrays were implanted in the hemisphere contralateral to the arm used in the experiment. FMAs consisted of 32 non-moveable monopolar platinum-iridium electrodes used for recordings, 2 electrodes served as grounds, and 2 electrodes served as references. Impedances of recording electrodes ranged between 300 and $600 \mathrm{k} \Omega$ at $1 \mathrm{kHz}$ before implantation and in 
vivo. For ground and reference electrodes this ranged between 5-200 k $\Omega$. Lengths of electrodes were between 1.5 and $7.1 \mathrm{~mm}$.

Before array implantation, a head post (titanium cylinder; diameter, $18 \mathrm{~mm}$ ) was implanted to train the animals to do the task while head-fixated. Array implantations followed when the animals were able to adequately perform the task in this way and were completely recovered from this initial surgery.

An MRI scan was done to locate anatomical landmarks like the arcuate and intraparietal sulci to guide the later array implantation. The monkeys were sedated (e.g., $10 \mathrm{mg} / \mathrm{kg}$ ketamine and $0.5 \mathrm{mg} / \mathrm{kg}$ xylazine, i.m.) and placed in the scanner (GE Healthcare $1.5 \mathrm{~T}$ or Siemens Trio 3T) in a prone position. Analysis of T1-weighted volumetric images of the brain and skull was done as described previously (Baumann et al., 2009). The arcuate sulcus of monkey S did not present a spur, but a small indentation in the posterior bank of the arcuate sulcus, about $2 \mathrm{~mm}$ medial to the knee, was revealed in the MRI image and treated as a spur. FMAs were placed lateral to that mark.

All surgical procedures were performed in sterile conditions while the monkeys were anaesthetized (e.g., induction with $10 \mathrm{mg} / \mathrm{kg}$ ketamine, i.m., and $0.05 \mathrm{mg} / \mathrm{kg}$ atropine, s.c., followed by intubation, 1-2\% isoflurane, and analgesia with $0.01 \mathrm{mg} / \mathrm{kg}$ buprenorphine). Heart and respiration rate, electrocardiogram, oxygen saturation, and body temperature were monitored continuously. Systemic antibiotics and analgesics were administered for several days after each surgery. The monkey was mildly hyperventilated to prevent brain 
swelling while the dura was open (end-tidal $\mathrm{CO}_{2}, \sim 30 \mathrm{mmHg}$ ). Mannitol was given as necessary. Training continued only after the monkeys were fully recovered from the surgery, which took about 2 weeks.

\subsection{Neural recordings and spike sorting}

All recordings were made in an electrically shielded room. Signals from the implanted arrays were amplified and digitally stored using a 128 channel recording system (Blackrock Microsystems; sampling rate $30 \mathrm{kS} / \mathrm{s} ; 0.3-7500 \mathrm{~Hz}$ hardware filter). Data was first filtered with a median filter (window length: $3.33 \mathrm{~ms}$ ) and the result was subtracted from the raw signal. Afterwards, the signal was low-pass filtered with a non-causal Butterworth filter $\left(5000 \mathrm{~Hz}\right.$; $4^{\text {th }}$ order).

Because the number of electrodes implanted in monkey B (192) exceeded the number of channels of the recording system, four different combinations of arrays were recorded that are shown in table 1. 
Table 1. Four different recording combinations of monkey B's arrays. Top row shows the areas represented in this combination. Lower rows show from which arrays neural signals were recorded. Black fields visualize which arrays were not used in that combination.

\begin{tabular}{|l|l|l|l|}
\hline F5 and AIP & F5 and M1 & M1 and AIP & F5, M1 and AIP \\
\hline F5 lateral & F5 lateral & F5 lateral & F5 lateral \\
\hline F5 medial & F5 medial & M1 lateral & F1 lateral \\
\hline M1 lateral & M1 lateral & M1 medial & M1 medial \\
\hline AIP lateral & M1 medial & AIP lateral & AIP lateral \\
\hline AIP medial & AIP lateral & AIP medial & AIP medial \\
\hline
\end{tabular}

Signals were recorded from all 128 channels of monkey S, but 58 of those channels did not show any neural activity on any of the recording days, were considered to be broken, and not processed any further. The total number of channels from monkey $\mathrm{S}$ that were processed was therefore 70. More than half of the channels on three arrays of monkey S were broken and many had an impedance of more than $5 \mathrm{M} \Omega$. This was attributed postmortem to a damaged cable, caused by a screw, between the electrode array and the connecter plug on the head post, due to a non-optimal design by the manufacturer.

To eliminate noise (e.g. movement noise) from the recorded signal, common components present on all channels of each array were identified and removed with principal component analysis (PCA) artifact cancellation (as described in Musial et al., 2002). To 
ensure that no individual channels were eliminated, PCA dimensions with any coefficient greater than 0.36 (with respect to normalized data) were retained.

Spike waveforms were extracted and sorted with a modified version of the offline spike sorter Wave_clus (Quiroga et al., 2004; Kraskov et al., 2009; Michaels et al., 2015). The Wave_clus toolbox first selects a combination of features of detected waveforms by calculating the wavelet transform, PCA and the original waveforms, selected by the degree of normality estimated by the Lillifors test. Second, based on the selected features, superparamagnetic clustering was applied, resulting in different cluster configurations called 'temperatures'. Low temperatures were best in separating broad waveforms, while high temperatures were best in separating narrow waveforms. We inspected the templates of the clusters at different levels of temperature, going from low to high temperature and selected templates that resembled known extracellular responses most closely. We then matched these templates with the unassigned waveforms, using an LDA (linear discriminant analysis) classifier in the toolbox.

After the semiautomatic sorting process, redetection of the average waveforms (templates) was done in order to detect overlaid waveforms (Gozani and Miller, 1994). The filtered signals were then convolved with the average templates, from high to low amplitude, to improve spike detection. To discriminate the waveform of the template from other waveforms, an LDA classifier, trained on the originally found waveforms, was used. After identifying the preferred waveforms, the shift-corrected template was obtained by up and down sampling and was subtracted from the filtered signal of the corresponding channel to 
reduce artifacts for detection of the next template. This procedure allowed a detection of templates up to an overlap of $0.2 \mathrm{~ms}$. After this process, waveforms were again visually inspected and similar looking waveforms on the same channel were fused together.

To determine if units should be classified as single- or multi-units, the waveform shape and inter-spike interval distribution of each was evaluated. A unit was only classified as singleunit when it met the following five criteria: 1 . Waveform shapes resembled known extracellular responses; 2 . Waveform clusters clearly separated in the projection of the first 17 features detected by Wave_clus; 3 . Detected spike waveforms homogeneously spread around the mean waveform; 4. The inter-spike interval histogram did not show a Poissonlike distribution; 5. Inter-spike intervals less than $2 \mathrm{~ms}$ were rare or absent. If a unit did not meet the first criteria or had an extremely low number of waveforms (typically fewer than 1000), it was classified as noise and not further analyzed. If most, but not all criteria were met, it was classified as an ambiguous unit. All other cases were classified as multi-units. This classification was only used for selecting example units and for creating the "bestchannel set' (see below). In all other analyzes, single-, ambiguous- and multi-units are treated equally.

\subsection{Data analysis}

All data was analyzed with custom written software in Matlab (MathWorks). 


\subsubsection{Behavioral data analysis}

In order to measure how well the monkeys were willing and able to perform the task, we calculated two different measures of performance: 1. Percentage of successful trials initiated after Cue epoch onset; 2. Percentage of successful trials initiated after Go signal. The first measure provides an indicator of the condition preference, while the second measure indicates how well the monkeys were able to perform the task.

Only successful trials were used in further analysis. The start and end time of each epoch was recorded, as well as events initiated by the monkey (Movement initiation and Touch). Reaction time (RT) was calculated as the time between Go and Movement initiation. Movement time (MT) was the time between Movement initiation and Touch. Gripping time (GT) was the time between Touch and Reward. RT, MT, and GT are also referred to as response times.

In order to have more homogenous results, trials with very long response times and cases where the monkey touched the sensors more than once were excluded from the analysis. Exclusion thresholds were the same for every session and were based on visually inspecting response time distributions of several sessions of both monkeys. The following trials were excluded: RT $>0.5 \mathrm{~s}, \mathrm{MT}>0.35 \mathrm{~s}$, and GT $>2.5 \mathrm{~s}$. In total, 961 out of 6669 trials (14\%) and 190 out of 2205 trials (9\%) were removed from the datasets of monkey B and S, respectively. 


\subsubsection{Grip force and electromyography analysis}

We recorded grip force in all sessions of monkey B and recorded surface electromyography (EMG) signals in 2 sessions. Grip force was not recorded in data sets of monkey $\mathrm{S}$ used in this study, but was recorded in a later session (session S130717) as a comparison. Grip force was smoothed with a Gaussian kernel $(\sigma=10 \mathrm{~ms}$, binsize $=2.5 \sigma)$ to reduce noise. EMG signals were band-pass filtered $\left(25-250 \mathrm{~Hz}, 6^{\text {th }}\right.$ order Butterworth), rectified, smoothed (Gaussian, $\sigma=10 \mathrm{~ms}$, binsize $=2.5 \sigma$ ), and normalized to the activity during the Fixation epoch. EMG activity was recorded from the ventral lower arm, dorsal lower arm, ventral upper arm, and dorsal upper arm where the activity most likely originated primarily from the flexor digitorum superficialis (FDS) muscle, extensor digitorum communis (EDC) muscle, biceps brachii muscle, and triceps brachii muscle, respectively.

\subsubsection{Peri-stimulus time histograms (PSTHs)}

Spike trains were stored with $1 \mathrm{~ms}$ resolution, smoothed (Gaussian, $\sigma=50 \mathrm{~ms}$, binsize= $\pm 2 \sigma$ ) and aligned around Cue epoch onset (400 ms before and $1300 \mathrm{~ms}$ after), Touch (500 ms before and $500 \mathrm{~ms}$ after), and Reward (1000 ms before and $200 \mathrm{~ms}$ after). These three PSTHs were concatenated and part of the neural activity before Reward was interpolated when it overlapped with the period after Touch. For example, when gripping time was exactly the Hold duration (as in most Low Force trials), the first 500 ms of the Reward alignment would completely overlap with the $500 \mathrm{~ms}$ after the Touch alignment. The linear interpolation therefore went from the end of the period after Touch (mean activity from 490 - 500 ms after Touch) until time point $y$ (mean activity from 0 - $10 \mathrm{~ms}$ after time point $y$ ) before Reward. Time point $y$ (ms) was calculated as follows: 
$y=\widetilde{G T}_{c}-T_{\text {BeforeReward }}+T_{\text {AfterTouch }}$

Where $T_{\text {AfterTouch }}$ is time after Touch (500 ms in this task), $T_{\text {BeforeReward }}$ is time before reward (1000 ms in this task) and $\widetilde{G T}_{c}$ is the median gripping time (ms) of condition $c$ (e.g. Precision Medium Force). To simplify, the formula can be written for this task as:

$y=\widetilde{G T}_{c}-500$

For example, when $\widetilde{G T}$ was $1320 \mathrm{~ms}$, activity was interpolated from $500 \mathrm{~ms}$ after Touch until 820 ms before Reward. $\widetilde{G T}_{c}$ was always at least $1000 \mathrm{~ms}$ long since this was the Hold time in this task. Therefore, $y$ was always at least $500 \mathrm{~ms}$. When $y$ was larger than $1000 \mathrm{~ms}$, no interpolation was done. This concatenated PSTH was used in further analysis. The part of the PSTH that was interpolated was not shown in the figures of the example units, but was used in the following analyzes.

\subsubsection{Cluster-based permutation test}

In order to calculate a statistical difference in firing rate between conditions at every time point and adequately deal with the multiple comparison problem, we used the clusterbased permutation test (Maris and Oostenveld, 2007). In short, we applied a two-way ANOVA in 10 ms steps along the PSTH and classified every time bin with $\mathrm{p}<0.01$ together with consecutive time bins with $\mathrm{p}<0.01$ as one cluster. This threshold of $\mathrm{p}<0.01$ is an 
arbitrary value to select the clusters and is not the final testing statistic. These clusters were classified separately for the p-values of the grip type main effect, force main effect, and interaction effect for the real data and for 1000 'shuffled' data sets. Each shuffled data set was created by randomly rearranging the condition labels of trials of the real data. The summed F-value was then calculated for every cluster. In each shuffled data set, the cluster with the highest summed F-value was selected to create a distribution of F-values (null distribution). This distribution was then compared to the summed F-values of each cluster in the real data, which was classified as significant when its summed F-value was higher than at least $99 \%$ of the F-values from the null distribution (i.e. $\alpha$-level= 0.01 ). An $\alpha$-level of 0.01 instead of the standard 0.05 was used to decrease the false positive rate to better emphasize the main effect. Because this method assumes that data from one time point depends on data from neighboring time points, it is important to not have huge time gaps between different alignments. We therefore used the interpolated data (from $1000 \mathrm{~ms}$ before Reward till 500 ms before Reward) to compute the clusters, but points indicating significance at these time points were not displayed in any of the figures because this significance is artificial.

\subsubsection{Medium force modulation index}

We calculated the relative modulation to the medium force condition compared to the lowand high force condition to visualize which condition is more similar in firing rate. We calculated this for every force tuned unit at every time point. We used the following equation: 
$y=\frac{f_{\text {Medium }}-f_{\text {Low }}}{f_{\text {High }}-f_{\text {Low }}}$

Where $y$ is the normalized firing rate (arbitrary units) for the medium force condition, $f_{\text {Medium }}$ is the firing rate $(\mathrm{Hz})$ for the medium force condition, $f_{\text {Low }}$ is the firing rate $(\mathrm{Hz})$ for the low force condition, and $f_{\text {High }}$ is the firing rate $(\mathrm{Hz})$ for the high force condition. This is calculated for every time point, for all units that are force tuned at that time. The median, first, and third quartile of the $y$ value of all units is subsequently calculated to visualize the population response.

\subsubsection{Demixed principal component analysis}

To get an overview of common patterns in PSTHs that explain most of the variance among all units, one can treat the response of a single unit as one dimension and then apply dimensionality reduction on the population data. Principal component analysis (PCA) is a common technique to reduce a high-dimensional dataset to only a few components that explain most of the variance. This technique can give basic insight in the structure of the dataset by showing components that explain most of the variance, but it does not explain the contribution of individual conditions to the variance. Since neurons show a mixed selectivity to task conditions, it is important to 'demix' this selectivity to understand the influence of task conditions on neural variance.

To do this, we applied a novel dimensionality reduction technique called demixed principal component analysis (dPCA) (Kobak et al., 2016) on the data, using freely available code: http://github.com/machenslab/dPCA. Like standard principal component analysis, 
dPCA extracts the components of a high-dimensional dataset that describe most of the variance, and calculates how much variance is explained by each component. However, unlike standard PCA, dPCA uses information about the task conditions (i.e. grip type, force, or interaction) to calculate the percentage of variance explained by each task condition or by changes over time (condition-independent). By not restricting the components to be orthogonal to each other, it then creates components that are primarily affected by a certain task condition. Additionally, this toolbox uses a linear classifier (stratified Monte Carlo leave-group-out cross-validation) to reveal at which time points the condition can be reliably decoded from an individual component.

Like for the cluster-based permutation test calculation, the PSTH described above was first down-sampled by a factor of 10 . The following parameters were used in the toolbox: the first 30 components were calculated, the number of repetitions used for optimal lambda calculation was 10 , the number of iterations used for cross-validation was 100 , and the number of shuffles used to compute the Monte Carlo distribution of classification accuracies expected by chance was 100 (as was done in Kobak et al., 2016). The time periods when actual classification accuracy exceeded all 100 shuffled decoding accuracies in at least 10 consecutive time bins were marked with colored lines on top of the figures showing the dPCA components.

\subsubsection{Best-channel set}

One of the challenges of analyzing multi-electrode array data, is how to treat neural data from different sessions. Unlike acute recordings, there is a likely chance to record from the 
same neuron in different sessions (Dickey et al., 2009). However, due to constant restructuring of the neural network, fluctuations in spike and noise amplitude, encapsulation of the electrodes, corrosion of the electrode tips, and micro-motions of the electrodes (Lee et al., 2005; Barrese et al., 2013; 2016), recordings can vary quite extensively from day to day. Because of this variation in the recordings, any measurable characteristic of a unit, such as waveform shape or spiking frequency, can be different in the next session, making it very difficult to objectively classify a unit as being the same as or different than a unit recorded on the same channel in another session.

It is therefore common to simply perform all analyzes on individual sessions and then average the result over all sessions. This works quite well when plenty of units were recorded per array and recordings did not vary much from session to session. However, this method could potentially bias your results since it gives more weight to stable units that were recorded in many sessions and to units that were recorded in sessions when the total number of recorded units was low. A simpler method that is much less affected by the number of units recorded in individual sessions is to pool all units together in one dataset. But this method will still be biased by the more stable units that are present in multiple different sessions.

To effectively deal with these issues, we created the 'best-channel set'. This is a dataset that consists of neural data from multiple sessions, but data from every electrode/channel is represented only once. For each channel in each session (recording) we counted the number of single-, ambiguous- and multi-units. We compared the number of unit types per 
recordings from the same channel and selected the session that had the best set of units for that channel, which meant a high amount of single-units, multi-units or spikes.

In detail, of the recordings from the same channel, the recordings were selected that had: 1 . The highest number of single-units; 2 . The highest number of ambiguous-units; 3 . The highest number of multi-units; 4 . The highest amount of spikes recorded from all singleunits; 5. The highest amount of spikes recorded from all ambiguous-units; 6 . The highest amount of spikes recorded from all units. At every step, the recordings were discarded that did not have the highest amount, until there was only one recording left (i.e. the single-unit count was the most important and the amount of spikes per units was only calculated when multiple sessions had the same number of the same unit types on that channel). In the end, the best-channel set consisted of 70 or 192 channels (for monkey S and B, respectively) that were taken from different sessions.

It is important to note that unit classification was purely based on waveform and interspike interval characteristics and not on task-responses, to not artificially increase the proportion of task-selective units. The best-channel sets had a much higher number of units than individual data sets, making it more suitable for population analyses. All population analyses were therefore performed on the best-channel sets of monkey B and monkey S. 


\section{Results}

This chapter presents the results obtained from the analyses described in the previous chapter. First the results are shown that describe the behavior of the monkeys whilst they performed the experimental task. Then the neural responses of the recorded single- and multi-units are described by analyzing the differences in firing rate. Finally, the characteristics of each investigated brain area are described after applying dimensionality reduction.

I present:

- The performance of both monkeys in the delayed grasping task (Chapter 3.1.)

- Reaction time, movement time, and gripping time (Chapter 3.2.)

- Force profiles while the monkeys performed the task (Chapter 3.3.)

- How muscle activity changed over the course of a trial, measured with electromyography (Chapter 3.4.)

- Details about the recording sessions (Chapter 3.5.)

- Responses of example single neurons to the task (Chapter 3.6.)

- Task tuning of the population of units recorded from each area (Chapter 3.7.)

- Results obtained with dimensionality reduction (Chapter 3.8.) 


\subsection{Task performance}

Both monkeys successfully learned the task. Figure 6 shows that more than $90 \%$ of the trials where movement was initiated after the Go signal (Movement trials) were correct. Performance was much lower when all trials were considered, because both monkeys would often break eye fixation during the Cue epoch when a condition appeared they liked less than other conditions. Furthermore, since a new trial would start automatically as long as the hand remained on the handrest button, many trials resulted in an error when the monkey was not moving the hands, but was constantly moving the eyes. We therefore believe that performance based on movement trials represented the monkey's capability to perform the task whereas performance based on all trials merely represented the monkey's motivation to perform a particular condition. High force trials were on average less preferred by both monkeys. Monkey B had a preference for whole-hand grip trials, while monkey $\mathrm{S}$ had a preference for precision grip trials.

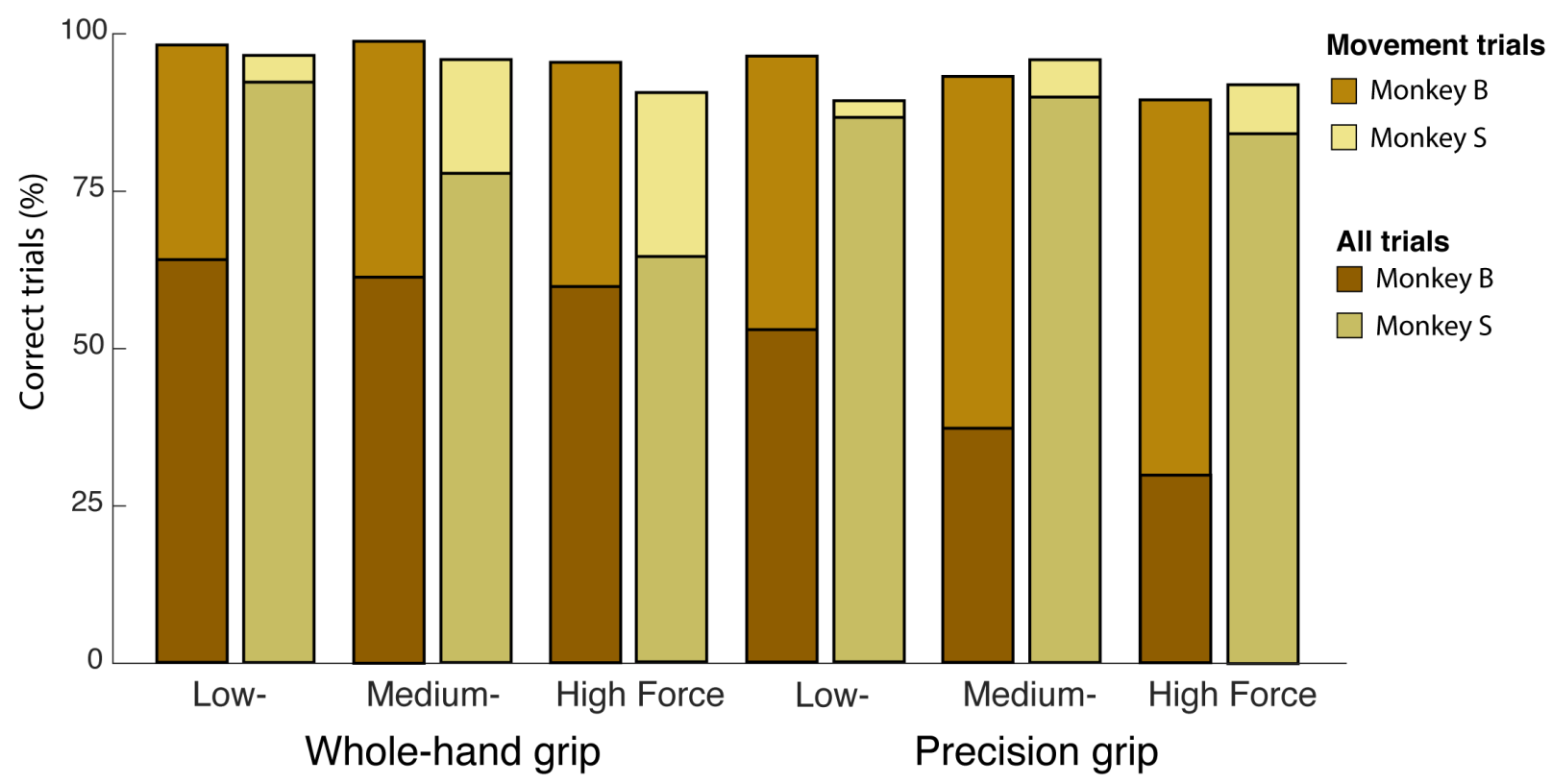


Figure 6. Task performance.

Percentage of correct trials of monkey B (brown) and monkey S (khaki) in each of the six task conditions. The total height of the bar indicates the monkey's performance in the fraction of trials when movement was initiated after the Go signal. The darker colored bars show the performance when all trials are considered.

\subsection{Response times}

We calculated how fast the monkeys responded to the Go signal (reaction time), how fast they moved from the handrest to the handle (movement time), and how long the monkeys were holding the handle until they obtained their reward (gripping time). Average reaction time (RT), movement time (MT) and gripping time (GT) for the selected trials are shown in Figure 7. Selected trials have a bit lower average response times than the raw data, since trials with very long response times (RT $>0.5 \mathrm{~s}, \mathrm{MT}>0.35 \mathrm{~s}$, and GT $>2.5 \mathrm{~s}$ ) were removed from the dataset (14\% of trials from monkey B and $9 \%$ of trials from monkey $\mathrm{S}$ ). The average reaction time of analyzed trials was $268 \pm 69 \mathrm{~ms}$ (mean $\pm \mathrm{SD}$ ) for monkey B and $257 \pm 72 \mathrm{~ms}$ for monkey S. Movement times differed between grip types in monkey S, with an average movement time of $121 \pm 14 \mathrm{~ms}$ for whole-hand grips and $192 \pm 43 \mathrm{~ms}$ for precision grips. For monkey B the movement times were longer than the movement times of monkey S and very similar for whole-hand grips (216 $\pm 32 \mathrm{~ms})$ and precision grips (220 $\pm 42 \mathrm{~ms}$ ). Gripping times were similar between monkeys, but depended greatly on the task condition, and especially on the force level. For monkey B, average gripping times were $1113 \pm 230 \mathrm{~ms}, 1467 \pm 295 \mathrm{~ms}$, and $1555 \pm 381 \mathrm{~ms}$ for whole-hand low force (WLF), whole-hand medium force (WMF), and whole-hand high force (WHF) conditions and 
$1085 \pm 215 \mathrm{~ms}, 1561 \pm 329 \mathrm{~ms}$, and $1718 \pm 360 \mathrm{~ms}$ for precision low force (PLF), precision medium force (PMF), and precision high force (PHF) conditions, respectively. For monkey S, these times were $1011 \pm 14 \mathrm{~ms}, 1403 \pm 304 \mathrm{~ms}, 1622 \pm 318 \mathrm{~ms}$ for WLF, WMF, and WHF conditions and $1151 \pm 312 \mathrm{~ms}, 1385 \pm 251 \mathrm{~ms}, 1539 \pm 332 \mathrm{~ms}$ for PLF, PMF, and PHF conditions, respectively. Minimum gripping time was equal to Hold time (1000 ms). Most low force trials had a gripping time of exactly 1 second, since the amount of force applied on the handle was always between 0 and $5 \mathrm{~N}$ at the moment of Touch.
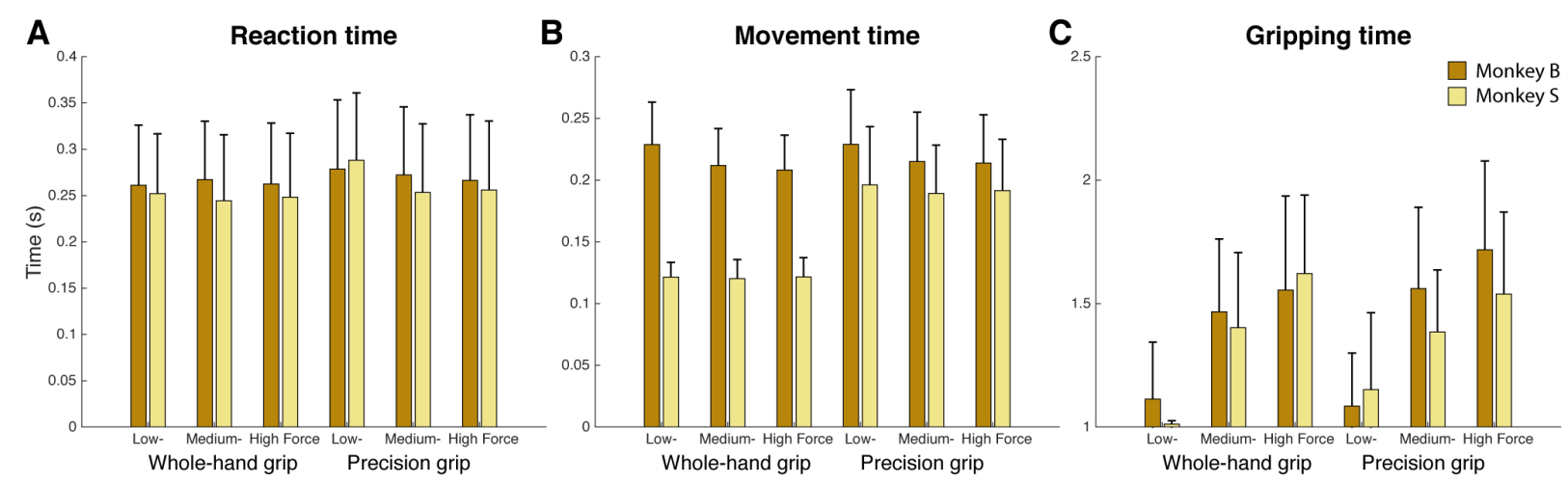

Figure 7. Response times.

A. Average reaction times of monkey B (brown) and monkey S (khaki) in each condition. Error bars indicate standard deviation. B. Same for movement times. C. Same for gripping times.

\subsection{Force profiles}

How force was applied in the different conditions is shown in Figure 8 and 10. It can be seen that most low force condition trials followed the same pattern; grip force rose quickly to about 2-4 N, stayed there for at least $1200 \mathrm{~ms}$ and then dropped to baseline. Only in a few cases in this condition was the initial amount of applied force above $5 \mathrm{~N}$ and then 
dropped down, which is best visible in Figure 8B. This initial overshoot was much more common for the medium- and high force conditions and especially for the whole-hand medium force condition of monkey B as can clearly be seen in Figure 8B and D.

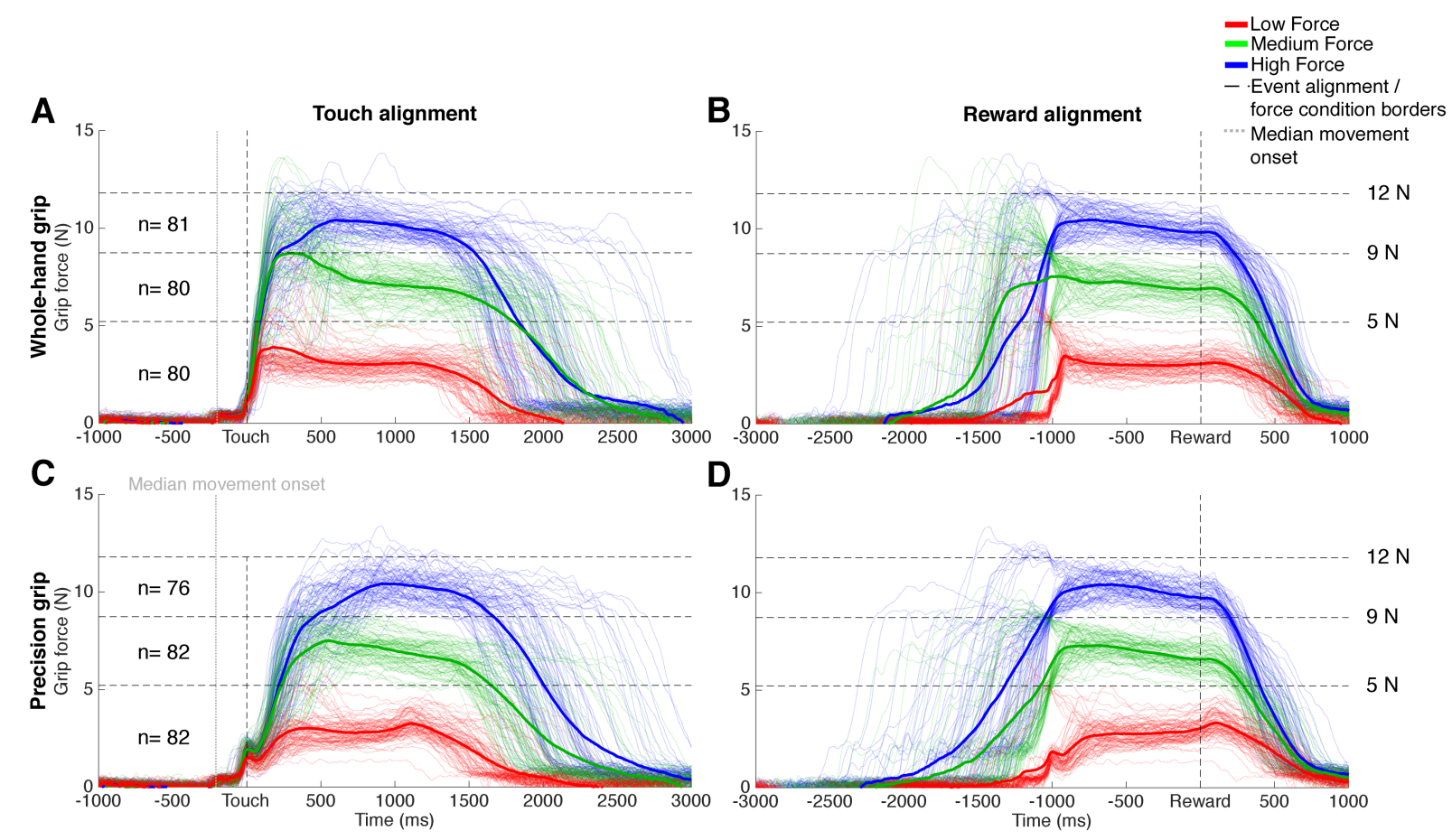

Figure 8. Force profile of session B150113.

Amount of force applied over time is shown for low force (red), medium force (green), and high force (blue) conditions for individual trials (thin transparent lines) and the average per condition (thick line) of session B150113 (monkey B). Dashed horizontal lines show the boundaries of the different force conditions corresponding to 5, 9, and $12 \mathrm{~N}$, as can be seen on the right side of the figure. $\mathbf{A}$ and $\mathbf{B}$ show the force profiles for the whole-hand grips. C and $\mathbf{D}$ show the force profiles for precision grips. A and $\mathbf{C}$ show the amount of grip force between $1000 \mathrm{~ms}$ before and $3000 \mathrm{~ms}$ after Touch. The number of trials per condition is shown on the left. A grey dotted line about $200 \mathrm{~ms}$ before Touch shows the median movement onset. Small artifacts due to voltage distortions are visible shortly after median movement onset (recorded voltage increased slightly due to release of the handrest buttons) and shortly after precision grip Touch (recorded voltage dropped slightly 
due to touching the precision grip sensors). B and D show the same data as in $\mathbf{A}$ and $\mathbf{C}$, but aligned at Reward, from $3000 \mathrm{~ms}$ before to $1000 \mathrm{~ms}$ after this alignment.

We can see in Figure 8A and 8C that the force profiles of the low force conditions diverge quickly after Touch from the two other conditions, while medium- and high force conditions typically started to diverge about $200 \mathrm{~ms}$ after Touch. In Figure 8 we even see that the average amount of force applied in the WMF condition is close to the upper border of this condition $(9 \mathrm{~N})$ and then drops down. A possible explanation for this behavior could be that monkey B did not fully use the information presented in the Cue epoch and waited for the reappearance of the force target (white square indicating the force condition; Figure 3A) to decide between the medium- or high force condition.

To test whether information from the Cue epoch was used, we measured if the differences between the force conditions were significant in the first $100 \mathrm{~ms}$ after Touch, i.e. when visual information from the reappearing force target could not yet influence the amount of force applied. We found in general a strong and significant difference between the force conditions (ANOVA, $\mathrm{p}<0.01$ ), but not between medium- and high force for the whole-hand grip (Tukey-Kramer test, $\mathrm{p}=0.77$ ) or precision grip (Tukey-Kramer test, $\mathrm{p}=0.99$ ). Similar p-values were found in other sessions, even when the average amount of force applied was different. This suggests that monkey B used the information of the Cue epoch to plan how much force to apply, but possibly did not distinguish between the medium- and high force conditions until it saw the force target reappearing. 
To investigate this further, we trained the monkey on a variation of the task where the force target did not reappear after Touch. Figure 9 shows the force profiles of the different conditions for this task and it can clearly be seen that monkey B first stayed within the medium force range and increased to the high force range after more than one second had passed without reward. Since this is a less efficient way to complete the task than by remembering the cues, we can conclude that monkey B did not distinguish between the medium- and high force conditions in the Cue epoch.

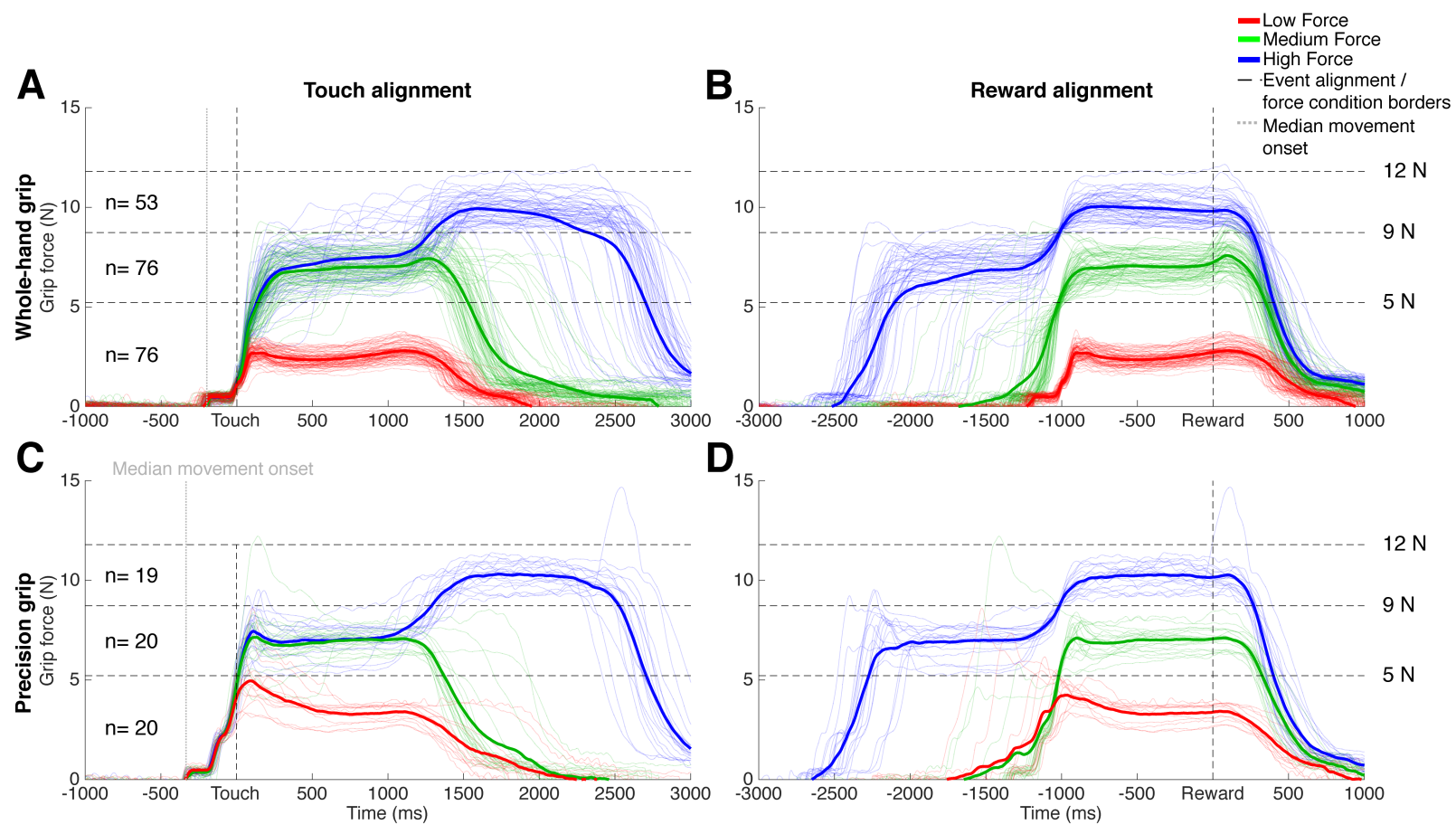

Figure 9. Force profiles of session B150513 (variation of the behavioral task)

Same as Figure 8, but for a session where the force target did not reappear in the Hold epoch. 
Monkey S appeared to have a similar strategy at first glance. It can be seen in Figure 10A and $\mathrm{C}$ that the grip force rarely came within the high force range before $150 \mathrm{~ms}$ after Touch, and the force increase is even slowed down for PHF trials (Figure 10C), which could indicate that the monkey waited for visual feedback before deciding between medium- or high force. Like monkey B, there was no significant difference in the first $100 \mathrm{~ms}$ after Touch between WMF and WHF applied grip force (Tukey-Kramer test, $\mathrm{p}=0.19)$. However, unlike monkey B, a significant difference in applied force was observed between PMF and PHF trials (Tukey-Kramer test, $\mathrm{p}<0.01$ ), which makes it very likely that monkey $\mathrm{S}$ distinguished between PMF and PHF cues in the Cue epoch. Because of these findings, and because monkey S was not trained on the variation of the task without target reappearance, it is hard to say how much this monkey distinguished between the mediumand high force cues during the Cue epoch. However, it was clear that the early response in low force trials was always much more different from the other two force conditions, than the differences between medium- and high force trials, suggesting that monkey S, like monkey B, also distinguished between the low force condition and the medium- and high force conditions combined. 


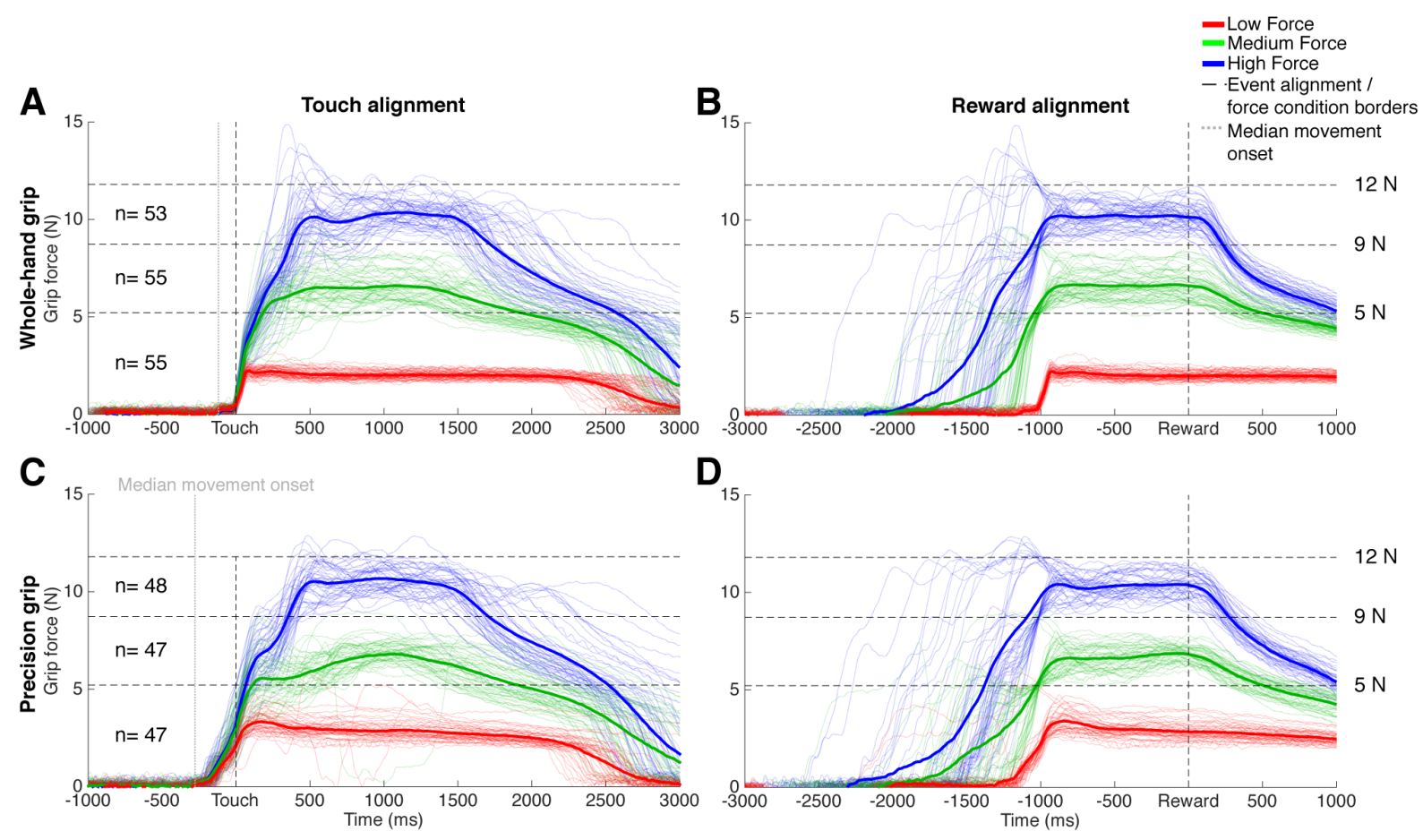

Figure 10. Force profiles of session S130717.

Same as Figure 8, but for a session of monkey S. This session was recorded after the sessions from which the neural signals were recorded.

\subsection{Electromyography}

In order to check that the animals were not initiating movements before the Go signal and to be able to relate neural activity to muscle activity in future analyses, we also recorded surface electromyography (EMG) signals on the moving arm of monkey B while he performed the task. Normalized EMG amplitude changes are shown for the flexor digitorum superficialis (FDS), the extensor digitorum communis (EDC), the biceps and the triceps in Figures 11A, B, C, and D, respectively. 

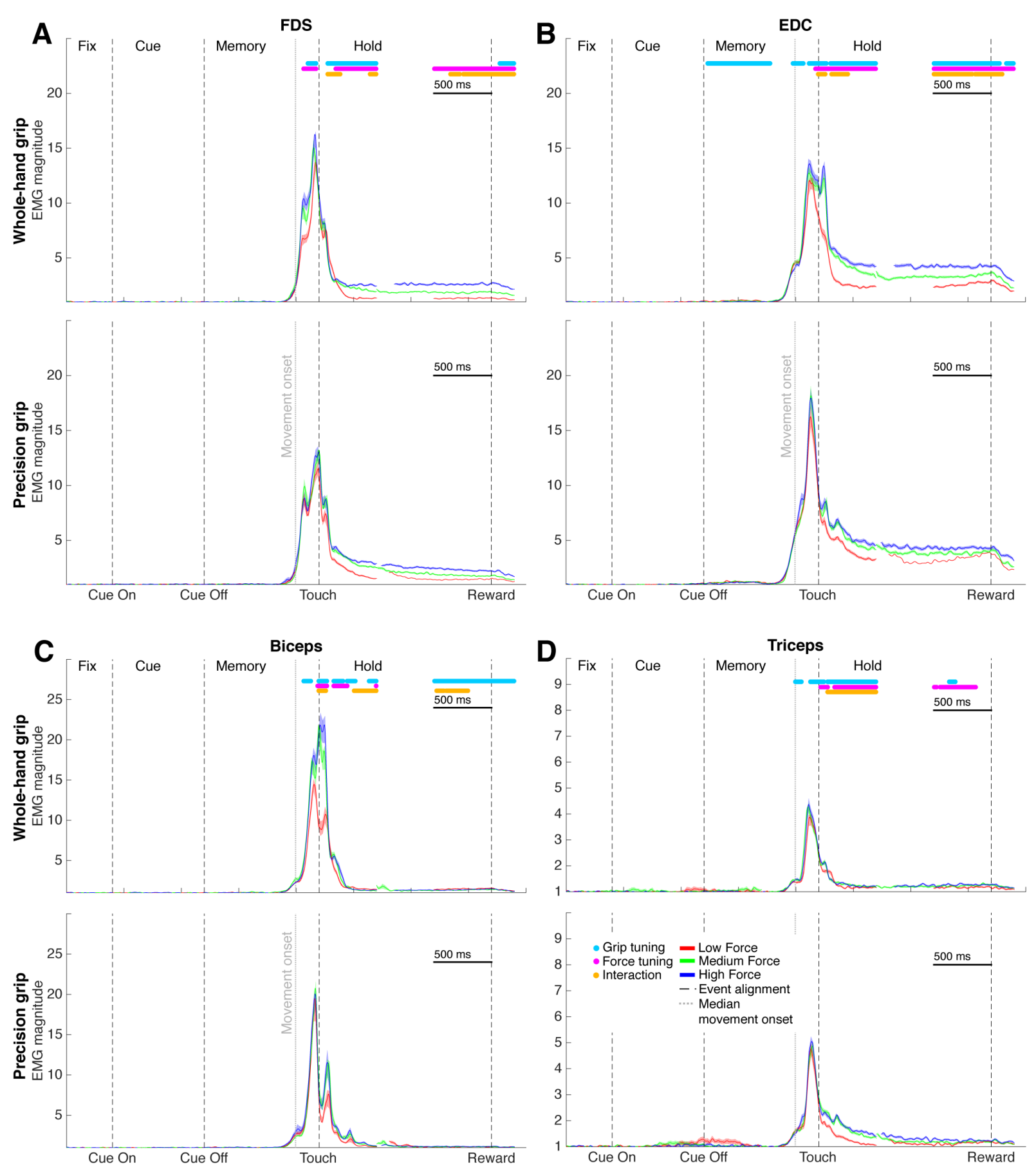

Figure 11. EMG signals of session B150219.

Average normalized EMG amplitudes of session B150219 (monkey B) are shown for low force (red), medium force (green), and high force (blue) conditions for the whole-hand grip (upper panels of subplots) and precision grip (lower panels of subplots). Shaded area around the lines represents the standard error of the 
mean. The signal is aligned at Cue On/Off, Touch, and Reward. These events are marked with vertical, black, dashed lines and their names are written on the x-axis of the lower panels. The vertical, grey, dotted line indicates the median movement onset. As is shown in bold text on top of each subplot, EMG signals were recorded from FDS (A), EDC (B), Biceps (C), and Triceps (D) muscle. Text below the name of the muscle, between the dashed lines, indicates the epoch names. Colored lines on top of each subplot shows the time intervals with significant difference in firing rate between grip type (cyan) or force (magenta) conditions, or when there was a significant interaction (gold) between grip type and force conditions (cluster-based permutation test, $\mathrm{p}<0.01)$. Scale: $500 \mathrm{~ms}$.

Different muscle activation patterns can be seen for the different grip types, but also for the different force conditions. EMG activity started to increase at around $400 \mathrm{~ms}$ before Touch and started to differ between the two grip types shortly after that. Peak activity occurred shortly before Touch in all muscles (with the exception of biceps activity in WMF and WHF conditions, Figure 11B) and then dropped to different levels, based on the task condition. As can be seen by the tuning significances, EMG activity typically started to differ between the conditions around movement onset (Figure 11D) or shortly afterwards (Figure 11A and C).

Notable is the significant grip type tuning (cluster-based permutation test, $\mathrm{p}<0.01$ ) in Figure 11B before movement onset, in the Memory epoch. This indicates that the EDC muscle is activated slightly different for the two grip types while the monkey is waiting for the Go signal. In the other session (B150225) with EMG data this was also observed and the EDC activity was even significantly different between force conditions in the Memory epoch. This early force tuning was probably only observed in the latter session because the 
EDC signals were recorded very clearly on that day (peak magnitude exceeded 30).

Nevertheless, the differences between the conditions are so small compared to the changes during movement that they are unlikely to have a major effect on the neural data.

We observed that early force tuning was always caused by the low force condition being different from the other two conditions and that, like the force profiles of monkey B (Figure 8), medium- and high force conditions did not differ in EMG activity until at least $150 \mathrm{~ms}$ after Touch. This observation fits with our earlier hypothesis that monkey B waited for the reappearance of the force target before deciding between the medium- and the high force condition.

\subsection{Session details}

We recorded neural data over 5 months in monkey B and over 2 months in monkey S. We selected 11 sessions of monkey B and 5 session of monkey $\mathrm{S}$ that had a high number of successful trials and a high performance for every condition. Table 2 gives an overview of the sessions selected for neural analysis. We analyzed more sessions of monkey B than of monkey S because we recorded additional sessions with M1 data and because monkey B had fewer F5 and AIP units in individual sessions than monkey S. Even though a substantial number of trials were excluded from each session due to long response times, every session still had at least 340 successful trials. Note that Table 2 does not include sessions B150513 and S130717 from which force profiles were extracted (Figures 9 and 10, respectively), but no neural data was analyzed. 
Table 2. Session details.

Number of successful trials, excluded trials, and recorded units in AIP, F5, and M1 in every session used in this study. When no units were recorded from the respective area in that session, it is labeled as NR (Not Recorded). In session B150219 we recorded with only one electrode array from F5 and one from M1, hence the number of units is lower in these areas. The number of successful trials ( $2^{\text {nd }}$ column) did not include the excluded trials ( $3^{\text {rd }}$ column). First letter of the session name indicates the monkey (B or S) and the following six numbers indicate the recording date.

\begin{tabular}{|c|c|c|c|c|c|}
\hline $\begin{array}{c}\text { Session } \\
\text { name }\end{array}$ & $\begin{array}{c}\text { Successful } \\
\text { trials }\end{array}$ & $\begin{array}{c}\text { Excluded } \\
\text { trials }\end{array}$ & AIP units & F5 units & M1 units \\
\hline B141021 & 591 & 138 & 33 & 45 & NR \\
\hline B141024 & 400 & 124 & 31 & 37 & NR \\
\hline B141028 & 344 & 87 & 30 & 46 & NR \\
\hline B141030 & 581 & 95 & 29 & NR & 53 \\
\hline B141104 & 498 & 99 & NR & 43 & 44 \\
\hline B141111 & 495 & 33 & 21 & 39 & NR \\
\hline B141120 & 590 & 77 & 27 & NR & 58 \\
\hline B141202 & 776 & 128 & 41 & 68 & NR \\
\hline B150113 & 484 & 63 & 27 & 50 & NR \\
\hline B150219 & 447 & 63 & 30 & 26 & 36 \\
\hline B150225 & 502 & 54 & 22 & 42 & NR \\
\hline S130612 & 356 & 34 & 60 & 62 & NR \\
\hline S130619 & 419 & 52 & 78 & 62 & NR \\
\hline S130620 & 360 & 35 & 67 & 67 & NR \\
\hline S130626 & 495 & 31 & 50 & 48 & NR \\
\hline S130704 & 385 & 38 & 80 & 77 & NR \\
\hline
\end{tabular}


To best analyze a large number of units from different areas, but without creating biased results, we created the best-channel set. This dataset contained a much higher number of single-, ambiguous-, and multi-units than individual sessions, as can be seen in table 3 . This dataset was used for all population analyses. 
Table 3. Number of single-, ambiguous-, and multi-units units per average session and in the bestchannel set.

Only sessions with data from two arrays per brain area were used to calculate the average number of units in that brain area. When no units were recorded from the respective area in that session, it is labeled as NR (Not Recorded).

\begin{tabular}{|c|c|c|c|c|}
\hline AIP & $\begin{array}{l}\text { Monkey B, } \\
\text { average } \\
\text { session }\end{array}$ & $\begin{array}{l}\text { Monkey B, } \\
\text { best-channel } \\
\text { set }\end{array}$ & $\begin{array}{l}\text { Monkey S, } \\
\text { average } \\
\text { session }\end{array}$ & $\begin{array}{l}\text { Monkey S, } \\
\text { best-channel } \\
\text { set }\end{array}$ \\
\hline Single-units & 11.2 & 47 & 24.0 & 56 \\
\hline $\begin{array}{l}\text { Ambiguous- } \\
\text { units }\end{array}$ & 3.1 & 10 & 6.2 & 11 \\
\hline Multi-units & 14.8 & 34 & 36.8 & 45 \\
\hline Total & 29.1 & 91 & 67.0 & 112 \\
\hline F5 & $\begin{array}{l}\text { Monkey B, } \\
\text { average } \\
\text { session }\end{array}$ & $\begin{array}{l}\text { Monkey B, } \\
\text { best-channel } \\
\text { set }\end{array}$ & $\begin{array}{l}\text { Monkey S, } \\
\text { average } \\
\text { session }\end{array}$ & $\begin{array}{l}\text { Monkey S, } \\
\text { best-channel } \\
\text { set }\end{array}$ \\
\hline Single-units & 14.9 & 62 & 29.6 & 57 \\
\hline $\begin{array}{l}\text { Ambiguous- } \\
\text { units }\end{array}$ & 4.0 & 9 & 5.6 & 7 \\
\hline Multi-units & 27.4 & 55 & 28.0 & 29 \\
\hline Total & 46.3 & 126 & 63.2 & 93 \\
\hline M1 & $\begin{array}{l}\text { Monkey B, } \\
\text { average } \\
\text { session }\end{array}$ & $\begin{array}{l}\text { Monkey B, } \\
\text { best-channel } \\
\text { set }\end{array}$ & $\begin{array}{l}\text { Monkey S, } \\
\text { average } \\
\text { session }\end{array}$ & $\begin{array}{l}\text { Monkey S, } \\
\text { best-channel } \\
\text { set }\end{array}$ \\
\hline Single-units & 18.0 & 40 & NR & NR \\
\hline $\begin{array}{l}\text { Ambiguous- } \\
\text { units }\end{array}$ & 3.0 & 8 & NR & NR \\
\hline Multi-units & 30.7 & 39 & $\mathrm{NR}$ & NR \\
\hline Total & 51.7 & 87 & NR & NR \\
\hline
\end{tabular}




\subsection{Single neuron responses}

A great variety of single neuron responses were found in every area. Figure 12 shows four example peristimulus time histograms (PSTHs) of such responses from F5 and AIP of monkey S (Figure 12A and B) and M1 and AIP of monkey B (Figure 12C and D). These examples show that significant differences in firing rate (cluster-based permutation test, $\mathrm{p}<$ 0.01) between grip types (grip tuning) and force conditions (force tuning) could appear at different moments in the task and that both tuning responses were present in every brain area. Although firing rate differences between conditions were highest in the Grasp and Hold epochs, significant tuning effects were often present in the Cue and Memory epochs, as can be seen in Figure 12A, B, and C. These neural responses have some resemblance with the EMG responses (Figure 11), especially the M1 neuron (Figure 12C), but the modulation before movement is much clearer and the low force condition often showed the highest activity (Figure 12B, C, and D), in contrast to EMG activity. Note that tuning responses could sometimes appear due to minute time differences in response onsets, as can be seen in Figure 12C, where the strong response to movement onset happened slightly sooner in the low force conditions.

Firing rates in medium- and high force conditions were usually much more similar to each other than to the firing rate in the low force condition (Figure 12A) and they were often identical to each other before the Hold epoch, especially in monkey B (Figure 12D and C). These findings are similar to what was observed in the force profiles (Figures 8 and 10) and in the EMG signals (Figure 11). 
There was great variety in which grip type or force condition evoked the highest firing rate (preferred condition) and this could change over the course of a trial, as can be seen in Figure 12B for grip force, and in Figure 12C for grip type. In most cases, the highest firing rate was found either for low- or high force. Medium force firing rate was usually between the other two force conditions. Whenever the medium force condition evoked the highest firing rate (dark magenta tuning line), it was usually only slightly higher than the low (Figure 12D) or high force condition (Figure 12A and C), with the latter case being observed more often in the visually inspected PSTHs (data not shown).

Significant interaction effects were common as well. This meant that force tuning was affected by grip type in a certain way. There are three main cases that evoked interaction tuning: grip force tuning was only present in one grip type (case 1), the same force conditions evoked highest and lowest firing rate, but they differed in magnitude between grip types (case 2), or the opposite force condition evoked highest firing rate in the other grip type (case 3). Cases 1 and 2 are usually hard to distinguish, but case 3 can easily be found where interaction, but not force tuning, was significant. All three cases can be observed in Figure 12.

One could also argue that grip type tuning depended on force tuning, but because hand shape did not appear to change significantly when more force was applied, we do not discuss this case. 
A
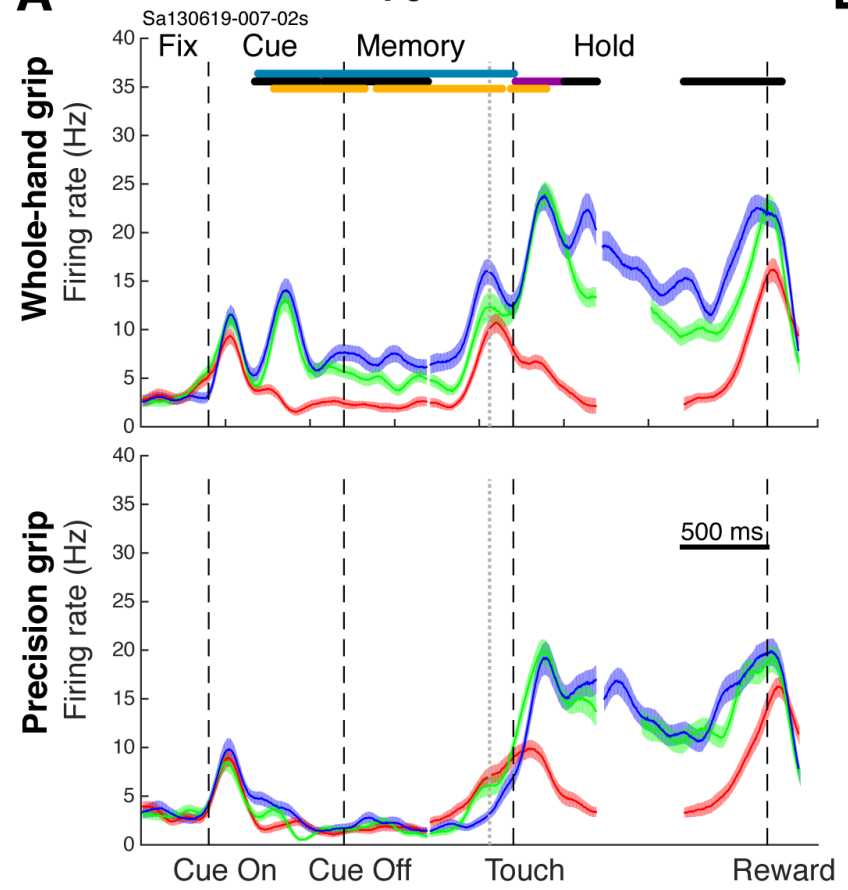

C
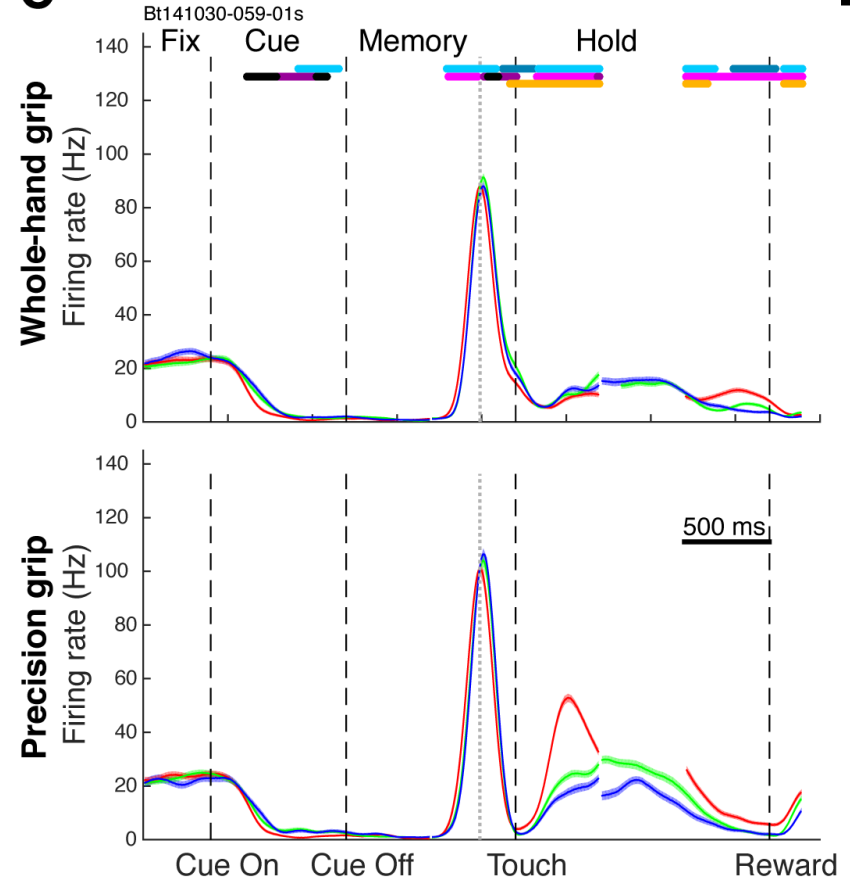

B
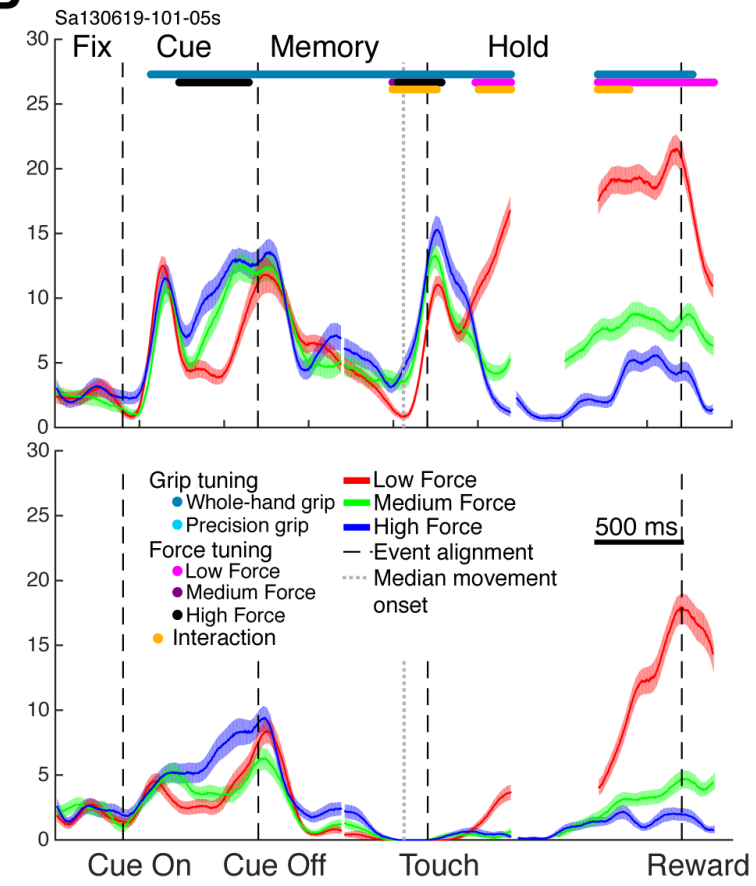

D
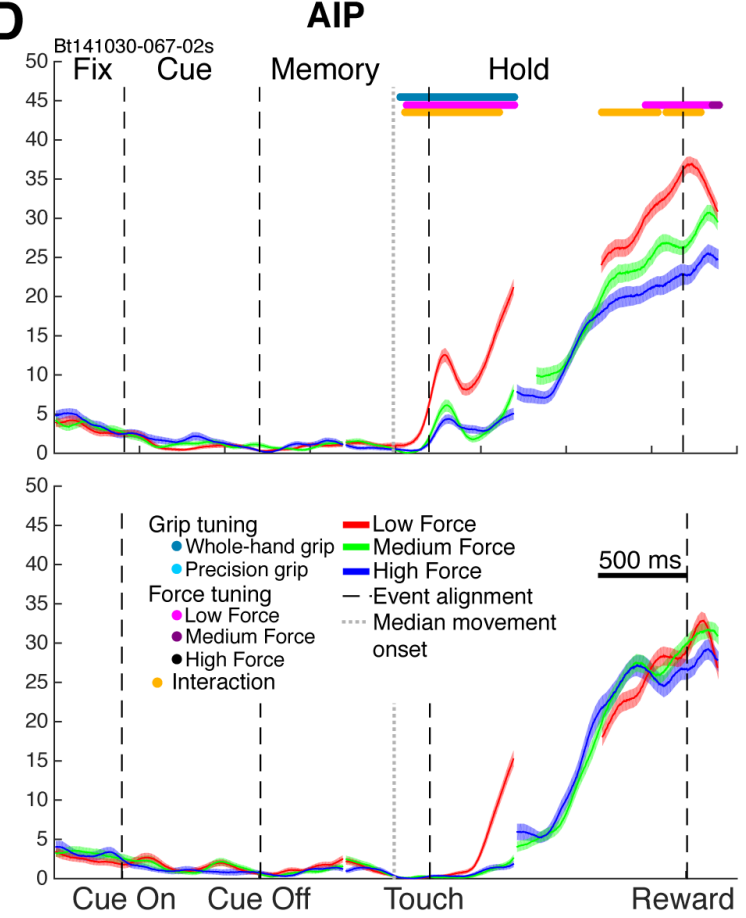

Figure 12. Examples of single neuron responses.

Convolved average firing rates of single neurons over time for low force (red), medium force (green), and high force (blue) conditions for whole-hand grip (upper panels of subplots) and precision grip (lower panels 
of subplots) trials. Shaded areas around the average firing rate curves represent standard error of the mean. The neural response is aligned at Cue On/Off, Touch, and Reward. These events are marked with vertical, black, dashed lines and their names are written on the x-axis of the lower panels. The vertical, grey, dotted line indicates the median movement onset. Bold text on top of each subplot indicates from which brain area this neuron was recorded. Small text on the top left of each subplot shows the name of the unit. Text on top of each subplot, between dashed lines, indicates the epoch names. Colored lines on top of each subplot (tuning lines) indicate time intervals with significant difference in firing rate between grip types (cyan), force (magenta and black), or with significant interaction (gold) between grip type and force (cluster-based permutation test, $\mathrm{p}<0.01$ ). Dark cyan lines indicate higher firing rate for whole-hand grips than precision grips at these time intervals; light cyan lines indicate the opposite. Light magenta lines indicate the highest firing rate for low force trials, dark magenta lines for medium force trials, and black lines for high force trials. Scale: 500 ms. A. Example neuron recorded in F5 of session S130619 (monkey S), B. Example neuron recorded in AIP of session S130619 (monkey S), C. Example neuron recorded in M1 of session B141030 (monkey B). D. Example neuron recorded in AIP of session B141030 (monkey B).

\subsection{Population tuning}

To investigate the common force tuning patterns in the neural population of the investigated brain areas, we plotted the time intervals with significant force tuning of the units in the best-channel set, sorted by their tuning onset (Figure 13-16).

\subsubsection{AIP tuning responses}

The first notable finding is that many AIP units were tuned for force at some point during the task (Figure 13). In monkey S, these tuning responses of AIP units started already in the Cue epoch and Memory epoch (Figure 13B). But in monkey B only very few AIP units 
showed force tuning in the Cue epoch and hardly any responded in the Memory epoch (Figure 13A). Force tuning in AIP was strongest for both monkeys after Touch. Figures 13C and D show the percentages of AIP units tuned for any of the task parameters at any time point. Even though monkey B had a much lower percentage of force tuned units during the Cue epoch $(<5 \%)$ than monkey S ( 20\%), both monkeys had between 20 and $30 \%$ of AIP units that were tuned for force during the Hold epoch.

Grip type tuning was much higher than force tuning in AIP, reaching values of about $50 \%$ around Touch (Figures 13C and D). Only in the last $500 \mathrm{~ms}$ of the Hold epoch, percentages of force tuning were similar to that of grip type tuning, especially in monkey S (Figure 13D). Furthermore, rises in percentages of units tuned for grip type after Cue onset also preceded that of force tuning in both animals. 

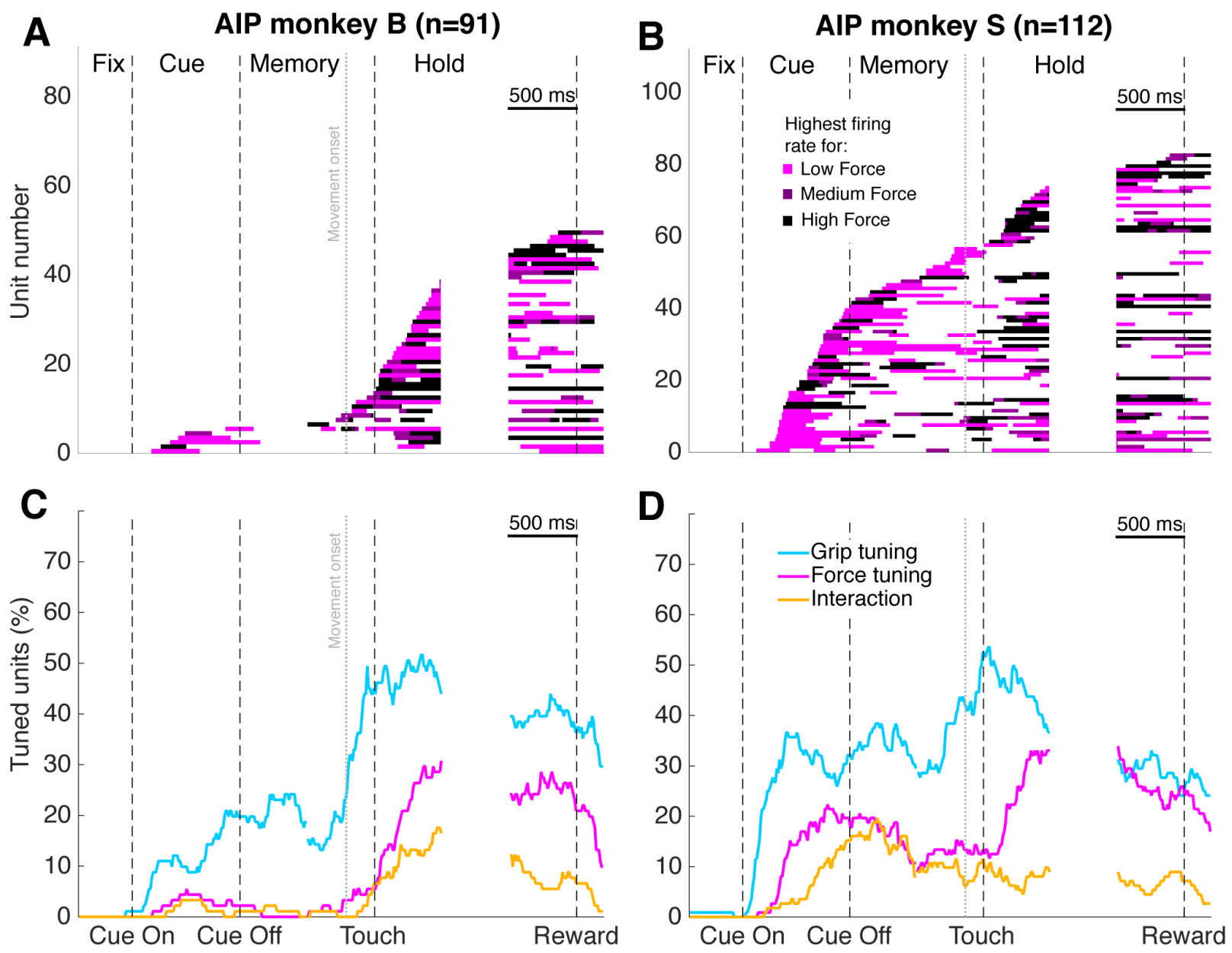

Figure 13. Population tuning in AIP.

$\mathrm{X}$-axis, alignments, epochs, and scale are the same as in figure 12. A. Force tuning lines of the AIP best-channel set of monkey B. Every magenta or black horizontal line represents time intervals when an AIP unit was tuned for force. Light magenta lines indicate the highest firing rate for low force trials, dark magenta lines for medium force trials, and black lines for high force trials. Force tuning lines are sorted by tuning onset, in ascending order. The total number of units in this dataset is shown on top of the figure between brackets in bold letters. B. Same as A, but for monkey S. C. Percentage of units tuned for grip type (cyan), force (magenta), or interaction (gold) at particular time intervals for monkey B's AIP best-channel set. D. Same as C, but for monkey S. 
Interaction tuning showed a similar pattern as force tuning, but did not rise as strongly after Touch. As described above, interaction tuning could coincide with force tuning, indicating that grip force tuning was only present in one grip type (case 1) or that force tuning differed in magnitude between grip types (case 2), but when it did not coincide with grip type tuning, force tuning was reversed in the other grip type (case 3). To investigate if the interaction component was dominated by one of these cases, we created the same figure with force and interaction tuning plus its combination Figure 14.
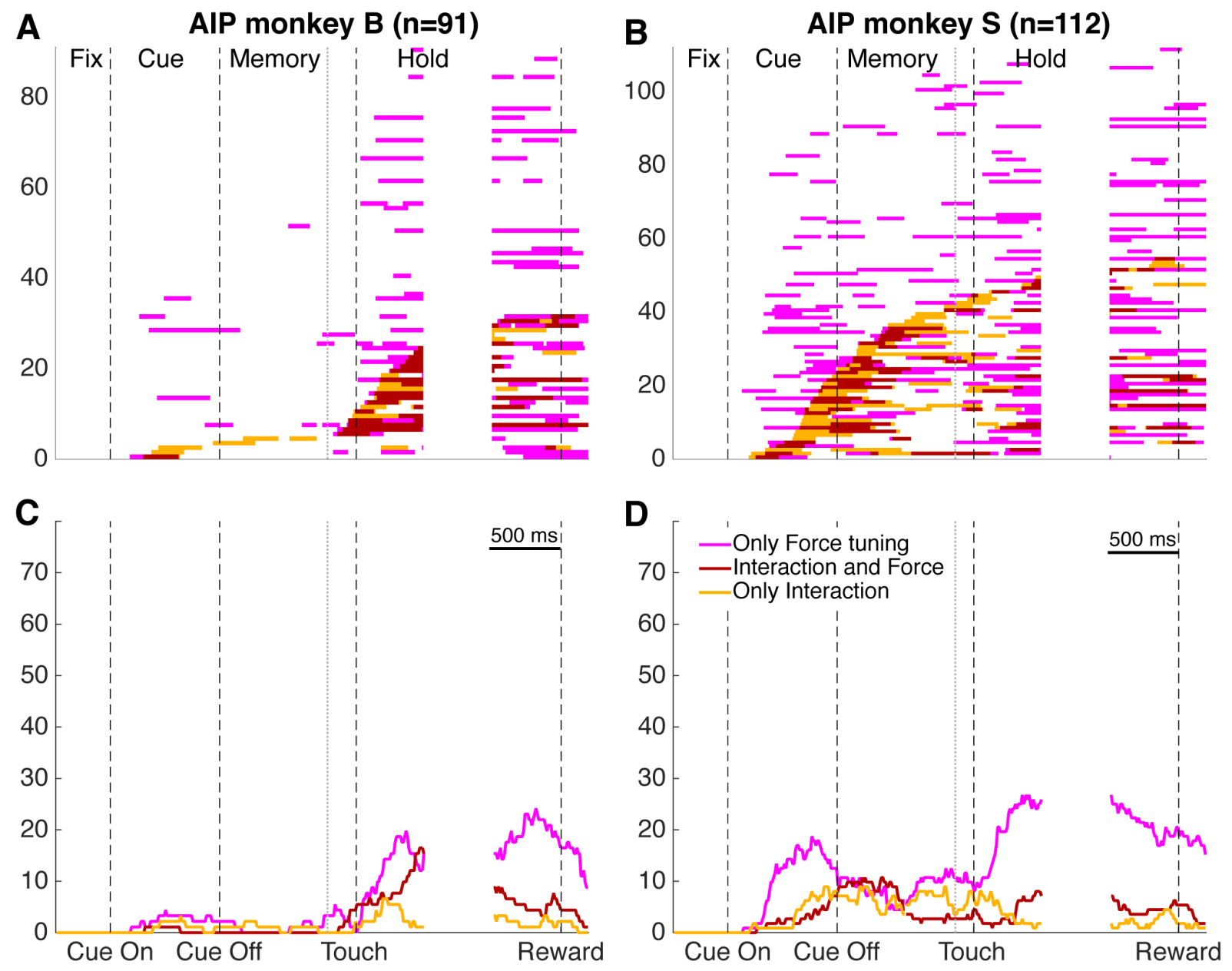

Figure 14. Force and interaction tuning in AIP. 
$\mathrm{X}$-axis, alignments, epochs, and scale are the same as in figure 12 and 13. A. Lines show the time intervals when a unit was tuned for only force (magenta), only interaction (gold), or both (dark red). Lines are sorted by interaction tuning onset, in ascending order. B. Same as A, but for monkey S. C. Percentage of units tuned for only force (magenta), only interaction (gold), or both (dark red) at particular time intervals for monkey B's AIP best-channel set. Note that adding the percentages of the dark red lines to the magenta or golden line, results into having the same magenta or golden line in Figure 13B, respectively. D. Same as C, but for monkey S.

We can see in Figure 14 that only in the middle of the Hold epoch there is clearly a higher percentage of units that are tuned for interaction and force simultaneously than for interaction alone, suggesting that case 1 and 2 are more common in this period, but all cases contribute to the interaction effect. Only-interaction tuning stayed at a similar level throughout the trial and did not rise during Hold. Areas F5 and M1 showed similar patterns

for interaction tuning (data not shown). These findings demonstrate that only a minority of units had reversed force tuning for the other grip type during the Hold epoch. The rise in interaction tuning observed in Figure 13 during Hold was interaction together with force tuning, hence there was no increase in reversed force tuning during this period. In conclusion, most units coding force, did this in the same way for whole-hand grip and precision grip.

\subsubsection{F5 tuning responses}

Similar tuning responses as in AIP (Figure 13) were observed in F5 (Figure 15), but higher percentages of units where tuned for force in this area. This was especially noticable in the 
Cue and Memory epochs of monkey B (Figure 13C and 15C). Also, we can see a rise in force tuning percentages in the middle of the Memory epoch, at the same time grip tuning percentages are rising, while this was not simultaneous in AIP. F5 responses in monkey B were similar to monkey S, only monkey S had higher percentages of force tuning, especially in the Cue and Memory epoch. Grip type tuning is similar in AIP and F5 until Touch, but dropped more sharply in F5 than in AIP. For most of the Hold epoch, force tuning percentages were higher than grip tuning percentages in F5, unlike in AIP. Interaction tuning in F5 followed the same pattern as in AIP. Altoghether, it seems that force is coded stronger in F5 than in AIP, even though this was not the case for grip type in this experiment. The stronger response to force before movement onset potentially reflects a bigger role for $\mathrm{F} 5$ in force planning. 


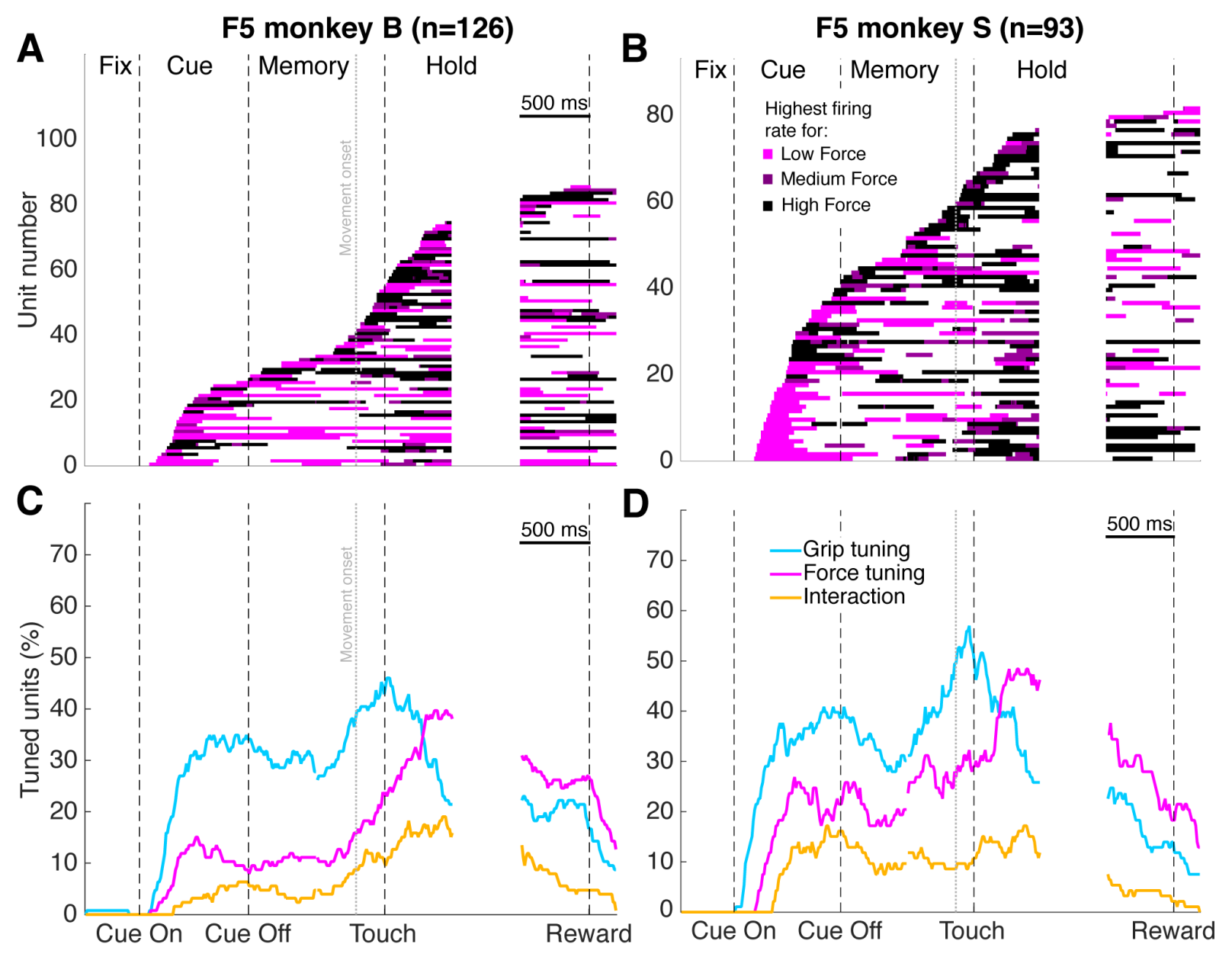

Figure 15. Population tuning in F5.

Same as Figure 13, but for the F5 best-channel set of monkey B and S.

\subsubsection{M1 tuning responses}

M1 units were only recorded from monkey B and their responses are shown in Figure 16.

As expected, many units in M1 were tuned for grip type and force, especially during

movement. However, even though the percentage of grip type tuned units was much higher in M1 than in F5 and AIP, the percentage of force tuned units was roughly the same as in F5 and only slightly higher than AIP. The force tuning percentages during Cue were lower than 
F5 and monkey S' AIP. Force tuning during Memory was neglectible, like in Monkey B's AIP. However, the rise in force tuning percentages occurred sooner in M1 than in AIP, and similar to F5. Like in AIP and F5, interaction tuning followed a similar pattern like force tuning, but in M1 interaction increased to a higher level in the Hold epoch than in AIP and F5. This shows a higher influence of grip type on force tuning of M1 units during movement.
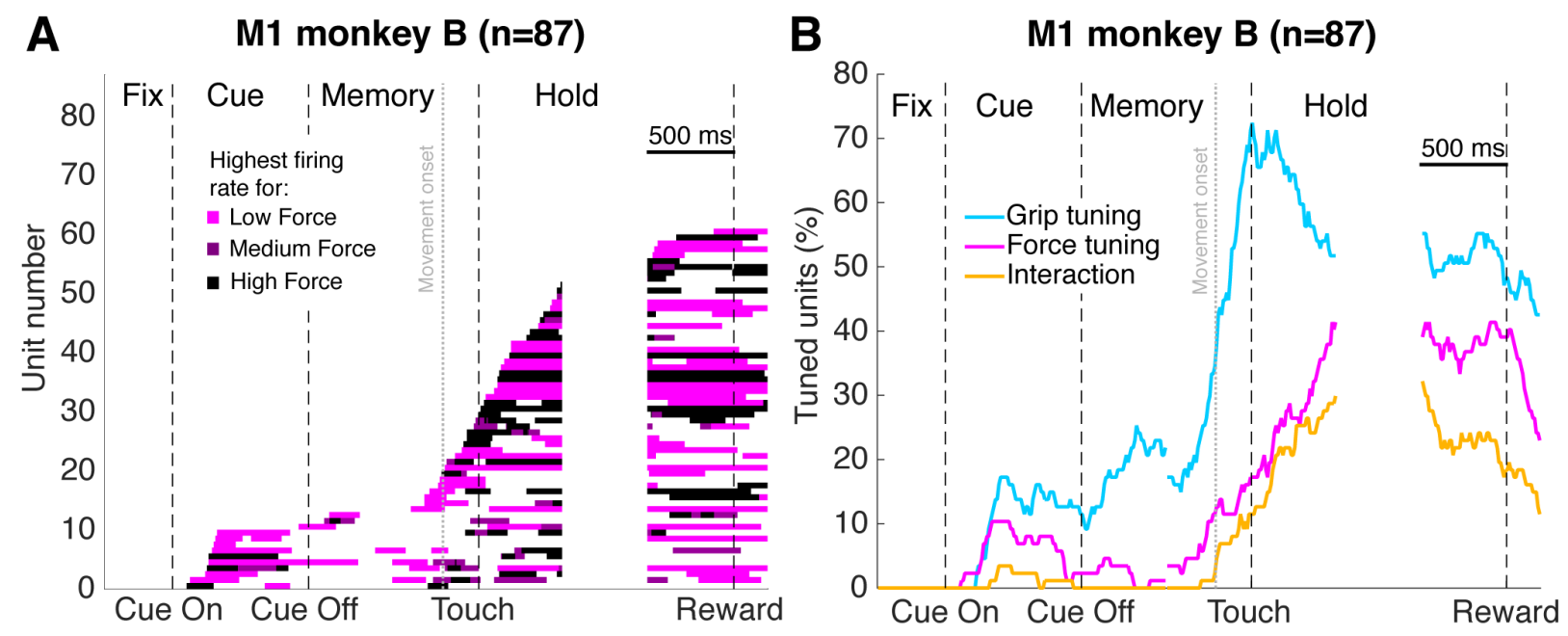

Figure 16. Population tuning in M1.

Same as Figure 13, but for the M1 best-channel set of monkey B.

\subsubsection{Preferred force condition}

Highest firing rate was usually evoked by the low- or high force condition. Periods where medium force evoked the highest firing rate were usually preceded or followed by periods when high force evoked the highest firing rate, indicating that both conditions evoked roughly the same firing rate, as can be seen in the example PSTH in Figure 12A. To visualize 
the similarity between the responses to the medium- and high force condition, we show the median firing rate for the medium force condition of all significantly force tuned units at a particular time point, normalized by the firing rate for the low- and high force (see methods) in Figure 17. We can see in each panel of this figure that the median values are, especially before Touch, much closer to 1 than to 0 , indicating that the firing rate for the medium force condition is much more similar to the firing rate for the high force condition as compared to the low force condition, in each investigated brain area. Furthermore, the median values were rarely higher than 1 and never below 0 , which indicates that, in the majority of cases, the firing rate for the medium force was between the firing rate for the low- and high force condition. 

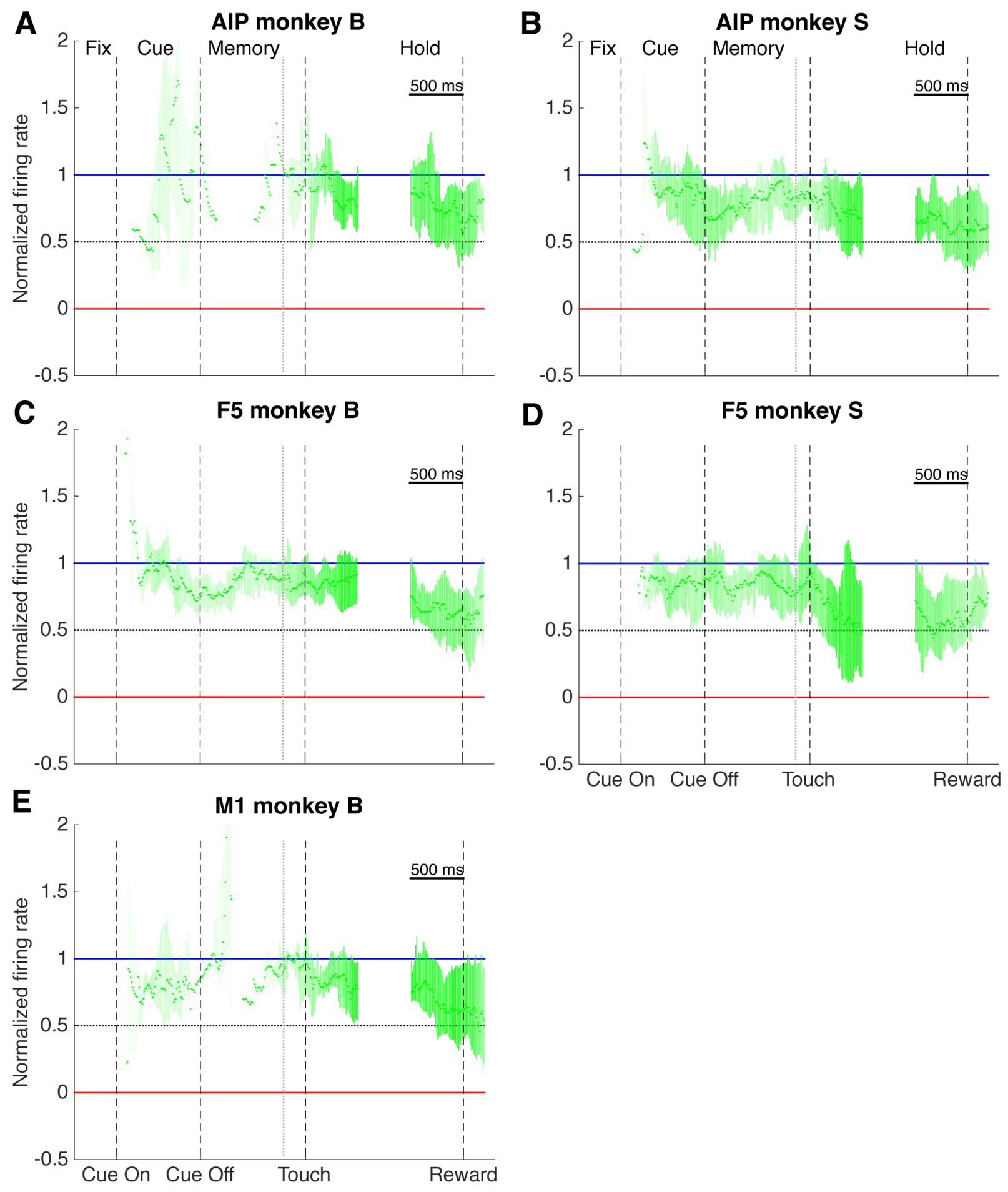

Figure 17. Medium force modulation index.

Firing rate for the medium force condition of significantly tuned units at a particular time point, normalized by the activity for the low- and high force conditions. A value of 0 means the firing rate is the same as in the 
low force condition (red line), a value of 1 means the firing rate is the same as in the high force condition (blue line), and a value of 0.5 means it is exactly between low- and high force (horizontal dotted line). Green dots indicate the median value of all units significantly tuned for force at that time point. The vertical green lines range from first till third quartile; transparency indicates the number of force tuned units (more transparent for fewer units). A. AIP best channel set of monkey B. B. AIP best channel set of monkey S. C. F5 best channel set of monkey B. D. F5 best channel set of monkey S. E. M1 best channel set of monkey B.

It seems to be more common that low force evoked the highest firing rate early in the Cue epoch, as can be seen in Figure 13B and 15B. On the other hand, around movement onset it is more common that high force evoked the highest firing rate, which can be seen clearest in Figure 15B. To gain an overview of which condition was more often preferred (i.e. which condition evoked the highest firing rate) by the recorded units, we show the percentages of tuned units that preferred the low force condition or either the medium- or high force condition in Figure 18. Medium force is grouped together with high force, because in the few cases that firing rate in this condition was higher than it was in the high force condition, it was usually only slightly higher (Figure 17). We can see in Figure 18 that both conditions are preferred equally often in AIP and M1 of monkey B (Figure 18A and E, respectively), but low force is preferred more often before movement onset and less often after movement onset in AIP and F5 of monkey S and F5 of monkey B (Figure 18B, D, and C, respectively).

The exact mechanisms for these patterns are unclear. It could be that activation patterns around movement onset are linked to the increasing activity of neurons in the corticospinal 
tract to increase muscle activity more in high force conditions (Figure 11-R9). However, we would then expect that especially in M1 the high force conditions evoke a higher firing rate, which was not observed (Figure 18E). Higher activity in low force conditions in the Cue epoch likely has to do with the conceptual understanding of this condition, and perhaps higher activity is linked to the higher motivation the monkeys had for performing this condition (Figure 6). 

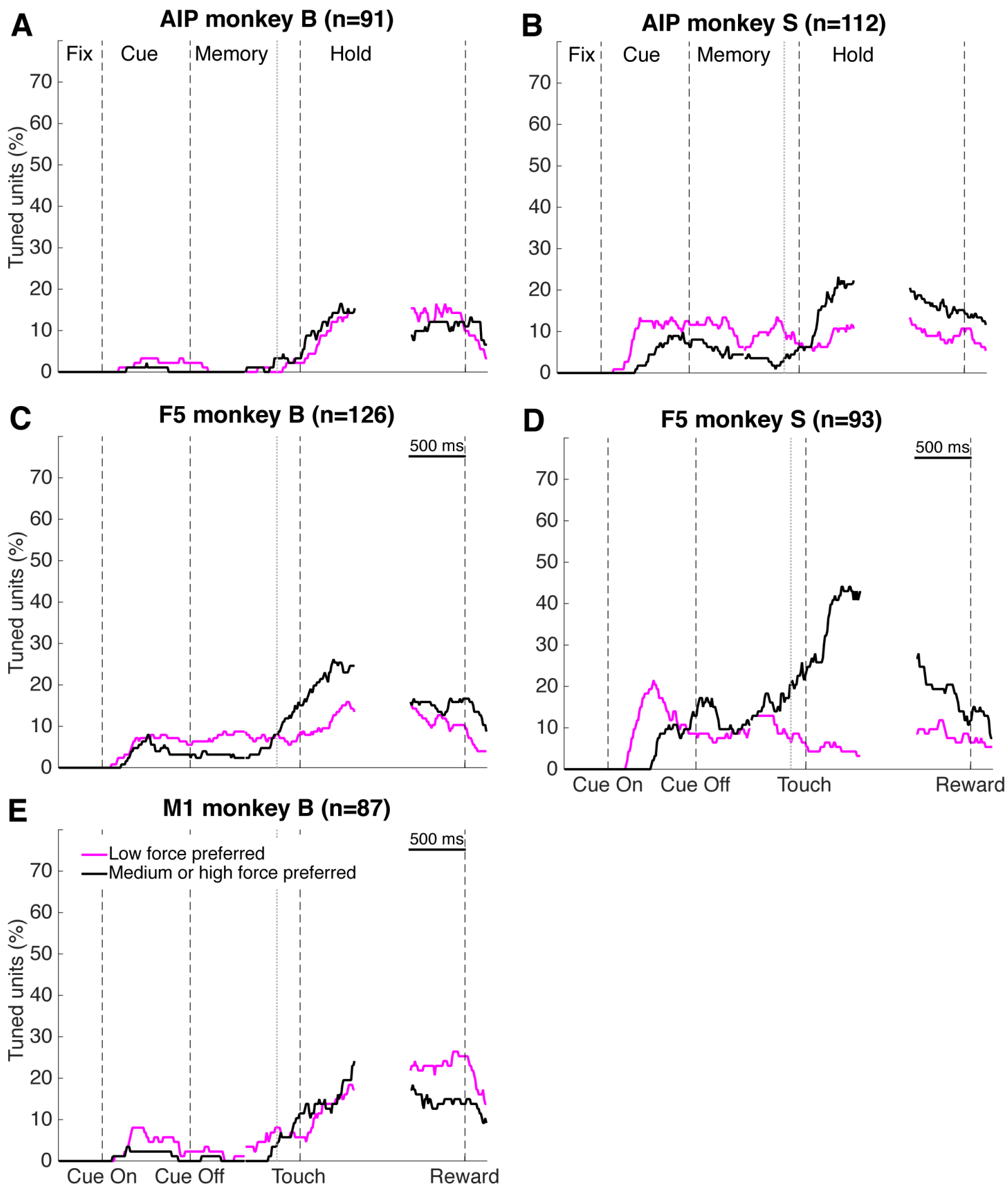

Figure 18. Percentage of preferred conditions.

Lines indicate the percentage of force tuned units that prefer the low force condition (magenta) or either the medium or high force condition (black). A. AIP best channel set of monkey B. B. AIP best channel set of 
monkey S. C. F5 best channel set of monkey B. D. F5 best channel set of monkey S. E. M1 best channel set of monkey B.

\subsubsection{Comparing best-channel set to average tuning}

All population results are based on the best-channel set to make sure that units recorded over multiple sessions cannot bias the results. However, since it is common to present the neural population response in electrophysiological studies by averaging the percentage of tuned units of every session, it is interesting to see how average tuning compares to results from the best-channel set. In Figure 19 we show average tuning percentages for the sessions used in this study. When we compare panels of Figure 19 to corresponding panels of figures 13, 15, and 16 (e.g. Figure 19A and Figure 13C), we can see that the results are almost identical. We can conclude from this that the results from the best-channel set correspond very well to the average response of individual sessions. 
A

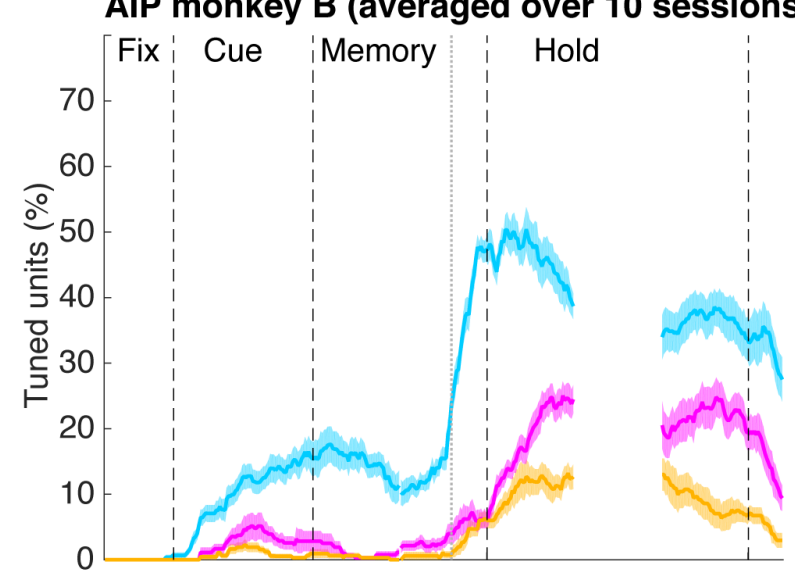

C

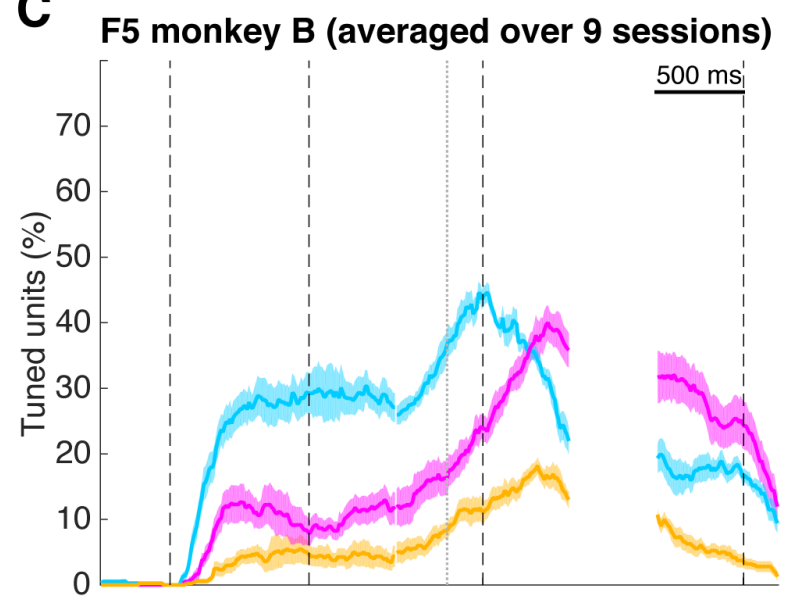

E M1 monkey B (averaged over 4 sessions)

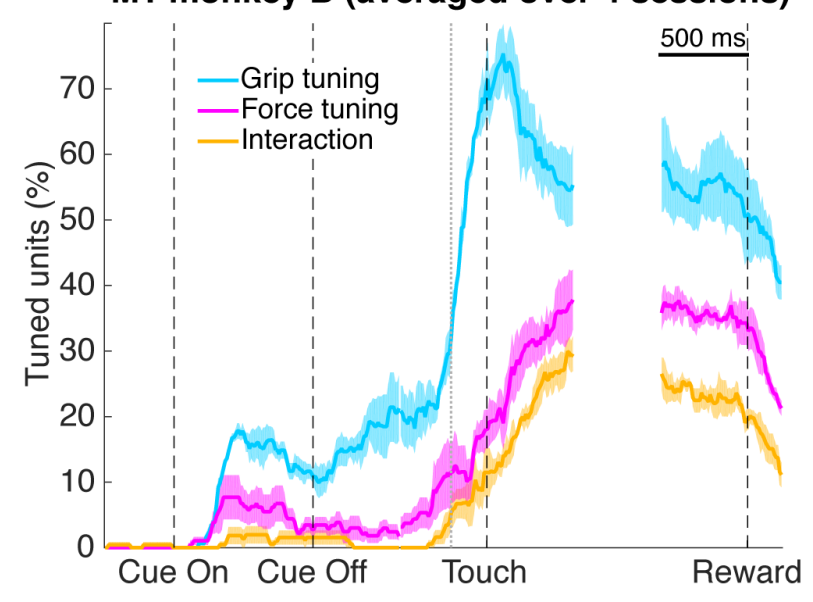

B AIP monkey S (averaged over 5 sessions)

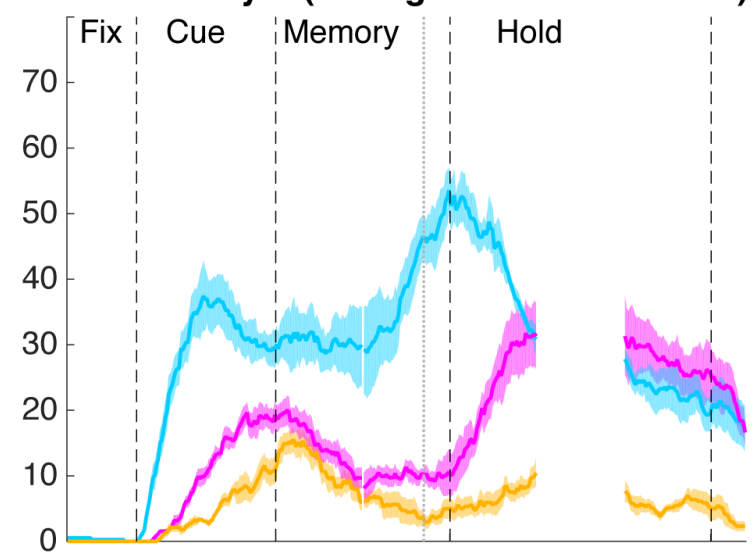

D F5 monkey S (averaged over 5 sessions)

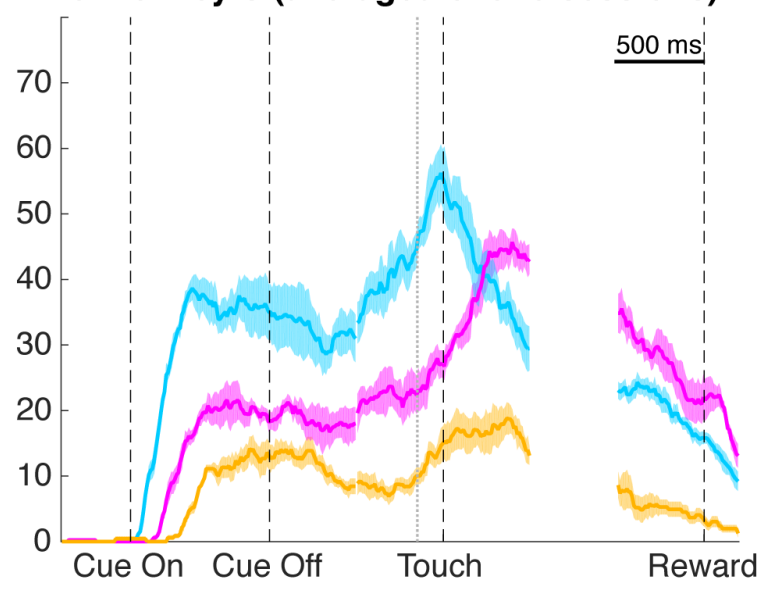

Figure 19. Average tuning over sessions.

Percentages of units tuned for grip type (cyan), force (magenta), and interaction (gold) averaged over sessions. Shaded areas represent standard error of the mean. A. Average tuning of the 10 sessions when AIP 
was recorded from monkey B. Similar to Figure 13C. B. Average tuning of the 5 sessions when AIP was recorded from monkey S. Similar to Figure 13D. C. Average tuning of the 9 sessions when F5 was recorded from monkey B. Similar to Figure 15C. D. Average tuning of the 5 sessions when F5 was recorded from monkey S. Similar to Figure 15D. E. Average tuning of the 4 sessions when M1 was recorded from monkey B. Similar to Figure 16B.

\subsection{Dimensionality reduction}

Tuning patterns in the different brain areas, as descried above, give a summary of how individual units responded to task parameters, but ignore the complex responses over the course of a trial that are visible in individual PSTHs (Figure 12). Furthermore, it also does not tell us how a significant difference in firing rate between conditions relates to the total change in firing rate over the course of a trial. For example, the neurons in Figure 12A and C both show significant force tuning before Movement onset, but for the F5 neuron (Figure 12A) this accounts for a much greater amount of the total variance than for the M1 neuron (Figure 12C). To get an overview of common responses in the different areas and to calculate how much of the variance is influenced by the task conditions, we applied a novel dimensionality reduction technique called demixed principal component analysis (dPCA) on the data (Kobak et al., 2016). Like standard principal component analysis (PCA), this method extracts the components that explain most of the variance and calculates how much variance is explained by every component. It also uses information about task conditions to calculate how much variance can be attributed to one of the conditions. With 
a linear classifier (stratified Monte Carlo leave-group-out cross-validation) it then calculates, for every time point, if conditions can be decoded from individual components. 
A

AIP monkey B
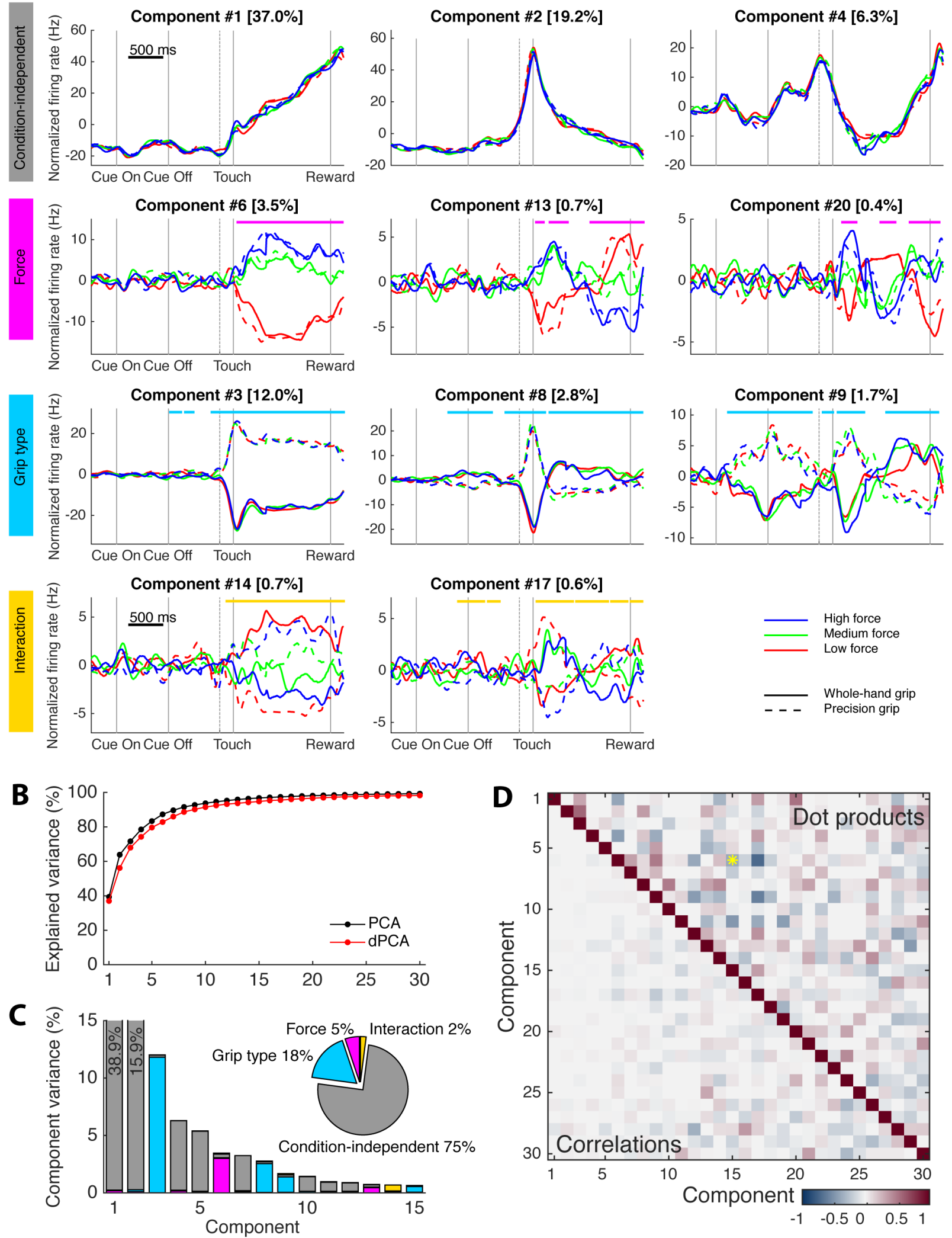
Figure 20. Demixed principal component analysis of AIP in monkey B.

A. Normalized firing rate over time of the first three demixed components of which variance is mainly attributable to condition-independent factors (top row), grasp force (second row), grip type (third row), or grip type and force interaction (fourth row). The red lines represent the firing rate in the low force condition, the green lines the medium force condition, and the blue lines the high force condition. Lines are solid for whole-hand trials and dashed for precision grip trials. A horizontal solid line on top of the components $\left(2^{\text {nd }}\right.$ row, magenta line; $3^{\text {rd }}$ row, cyan line; $4^{\text {th }}$ row, golden line) shows the time periods when the respective task parameter can be reliably decoded (classification accuracy exceeded all 100 shuffled accuracies in at least 10 consecutive time bins). Task events Cue onset, Cue offset, Touch, and Reward are marked as vertical, grey, solid lines. The vertical, grey, dotted line indicates the median movement onset. A timescale of $500 \mathrm{~ms}$ is visible in the top and bottom plot of the first column. B. Total amount of variance explained for the number of components used in the analysis for PCA (black) and dPCA (red). C. Barplot shows the amount of variance explained by the first 10 components. Colors show the proportion of variance explained by conditionindependent factors (grey), grip force (magenta), grip type (cyan), and interaction (gold). D. Upper-right triangle shows dot products between all pairs of the 30 demixed principal axes, yellow asterisks indicate when pairs are significantly and robustly non-orthogonal (Kendall correlation, $\mathrm{p}<0.001$ ). Bottom-left triangle shows correlations between all pairs of the 30 demixed principal components.

\subsubsection{Demixed components and explained variance in AIP}

Figure 20A shows 11 of these demixed components from the AIP best-channel set of

monkey B. The first and largest component, explaining 37.0\% of neural variance, shows an increase in firing rate that starts at movement onset and continues until after Reward. Component 2 shows an increase in firing rate shortly before median movement onset, peaking around Touch and then dropping again during the Hold epoch. The third component is strongly affected by grip type. A thick cyan line on top of the plot of component 3 shows that grip type could be reliably decoded at several points in a trial. 
First, there is a relatively small, but decodable, difference in firing rate shortly after Cue offset, lasting about $250 \mathrm{~ms}$. Then shortly before median movement onset, these firing rates diverge again for the grip type and this can be decoded for the remainder of the trial. These first three components show that already $68.2 \%$ of the neural variance in AIP of monkey B can be explained by: 1. steady increase during Hold, 2. phasic response around Touch, 3 . phasic-tonic response to grip type during movement.

Two other grip type dominant components are shown in Figure 20. These components explain much less of the variance than component 3 , but also in these components could grip type be reliably decoded during the Hold epoch and, additionally, during most of the Cue and Memory epoch.

The largest component that is mainly influenced by the force conditions is component 6 . This component shows a divergence of the force conditions directly after Touch, continuing until the end of the trial. As was observed in the example unit of Figure 12D, low force diverged first from the other two force conditions, and the medium- and high force conditions diverged later. Similar patterns were observed in component 13 and 20, although force was not decodable for the entire Hold epoch in these components. Note that force could not be decoded before Touch from any of these components.

A few small components were influenced by both grip type and force in a way that could not be disentangled (interaction). Two of these components are shown in Figure 20, they 
show that firing rate during the Hold epoch was similar for some conditions that differed both in grip type and force.

How much each of the four factors (grip type, force, interaction, and conditionindependent) contributed to the variance in the 30 extracted components, is shown in the pie chart of Figure 20C (only the first 10 components are shown in the bar plots). The highest contributor to the variance is condition-independent, responsible for $75 \%$ of the variance. This is not a surprising finding as this was already found in many other in vivo electrophysiological experiments (Kobak et al., 2016), but is nevertheless important to note, while it is often ignored in other methods, such as tuning analyses. The second highest contributor is grip type, responsible for $18 \%$ of the variance. Force is a much smaller contributor, but still explains $5 \%$ of the variance. Interaction is the smallest contributor, explaining only $2 \%$ of the variance. Because variance explained by interaction is much smaller than what is explained by grip type or force, we can conclude that most of the grip type and force information in monkey B's AIP can be extracted separately.

Figure 20B shows that dPCA explains almost as much variance as PCA, indicating that little information is lost with this method. Figure 20D shows in the upper-right triangle that, even though dPCA does not enforce orthogonality between the components, only one pair (marked with a yellow asterisk) was significantly non-orthogonal. The bottom-left triangle in Figure 20D shows the correlations between pairs of demixed components, most of them are close to zero. 
Though similar to monkey B, some different observations were made in AIP of monkey S (Figure 21). Most importantly, grip type is represented much stronger in this dataset, explaining $30 \%$ of the component's variance, and components 2,7 and 9 show that this could already be decoded early in the Cue epoch. Furthermore, force conditions could be decoded in the Cue and Memory epoch (most clear in component 11), which was not found in monkey B.

The condition-independent components described for monkey B are also found in monkey S, only now the phasic response to Touch is a larger component than the steady increase during Hold. Component 2, the largest grip type component, looks quite different from the grip type components found in monkey B, but component 9 of monkey $\mathrm{S}$ bears some similarity with component 8 of monkey B. Force component 6 of monkey $S$ is also similar to component 6 of monkey B, only component 11 of monkey S clearly stands out with Cue and Memory force effects. In Figure 21D we can see more pairs that are significantly nonorthogonal than in Figure 20D. However, the great majority of pairs were still close to orthogonal. 
A AIP monkey S
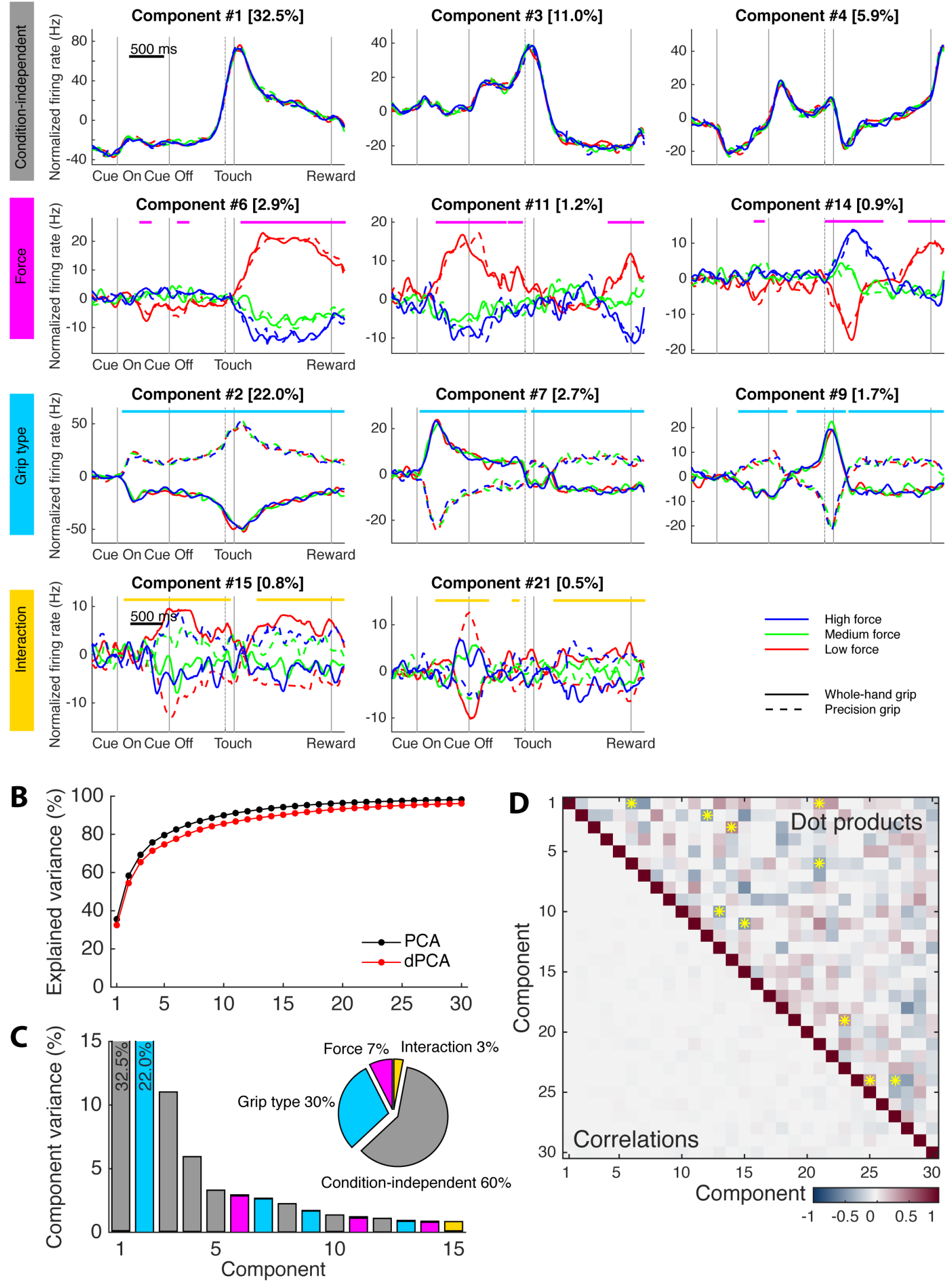
Figure 21. Demixed principal component analysis of AIP in monkey S.

Same as Figure 20, but for the AIP best-channel set of monkey S.

\subsubsection{Demixed components and explained variance in F5}

We saw that the tuning patterns of F5 (Figure 15) hold some similarities with AIP (Figure 13), especially with AIP of monkey S. We can make a similar observation when we compare the components revealed by dPCA of F5 (Figures 22 and 23) and AIP (Figures 20 and 21). However, there are two important differences to note. First, there is a strong coding of force in the Cue and Memory epoch, as is clearly visible in component 6 of Figure 22 and component 15 of Figure 23. Second, even though the total percentage of variance in the components contributable to force is similar to AIP, the variance contributed by grip type is much lower in F5. This does not automatically mean that AIP contains more information about grip type, but it means that the amount of variance contributing to force in relation to grip type is higher in F5. 
A

F5 monkey B
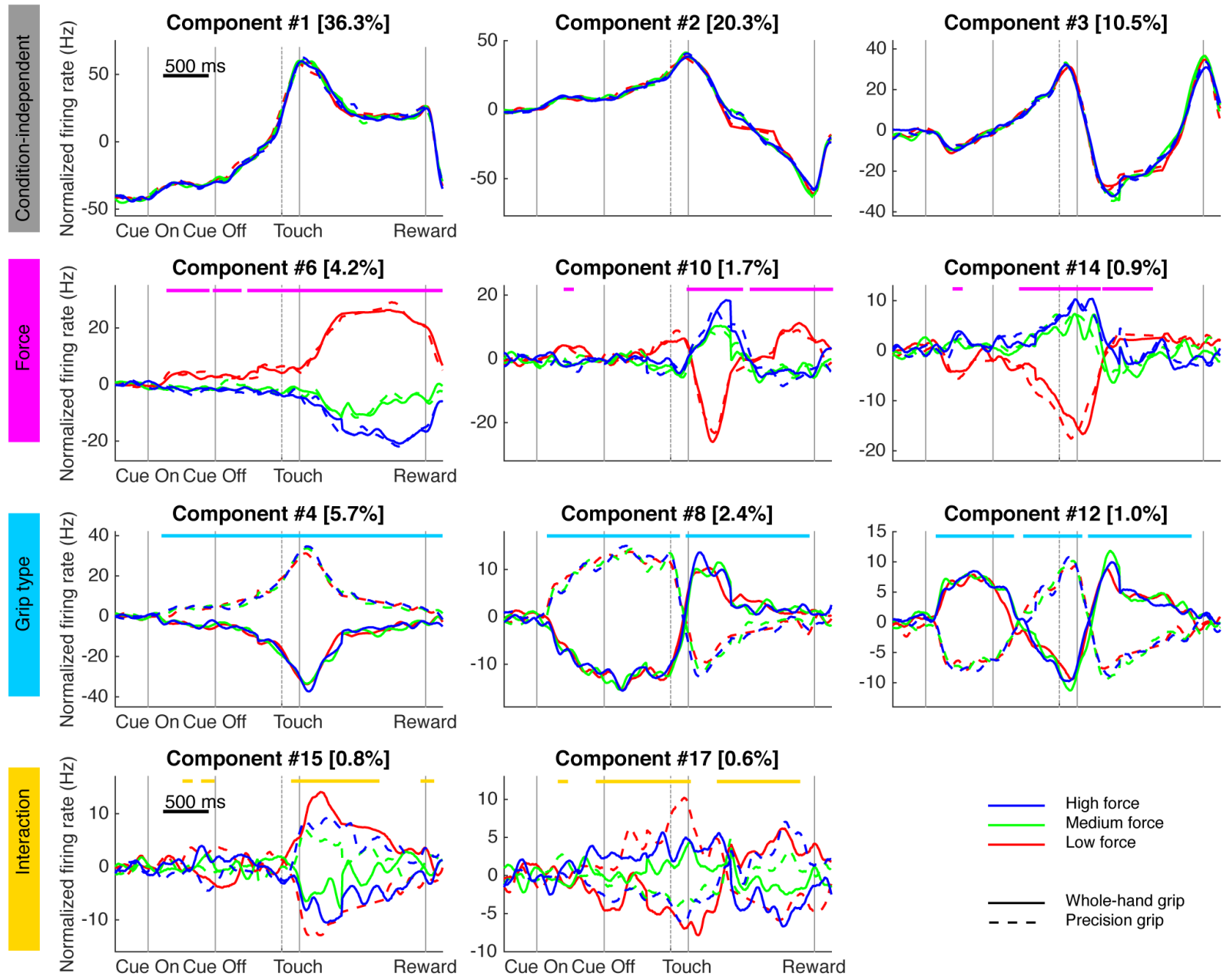

B

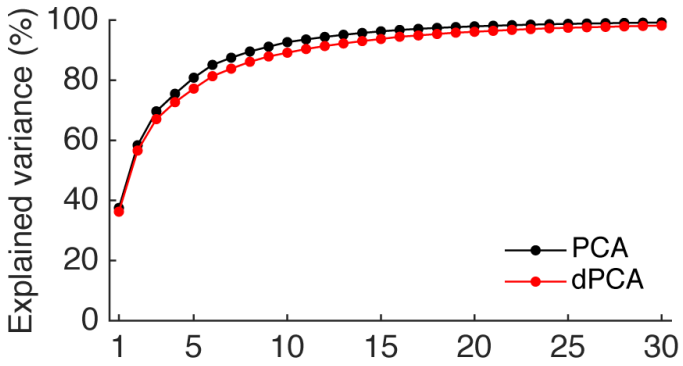

C
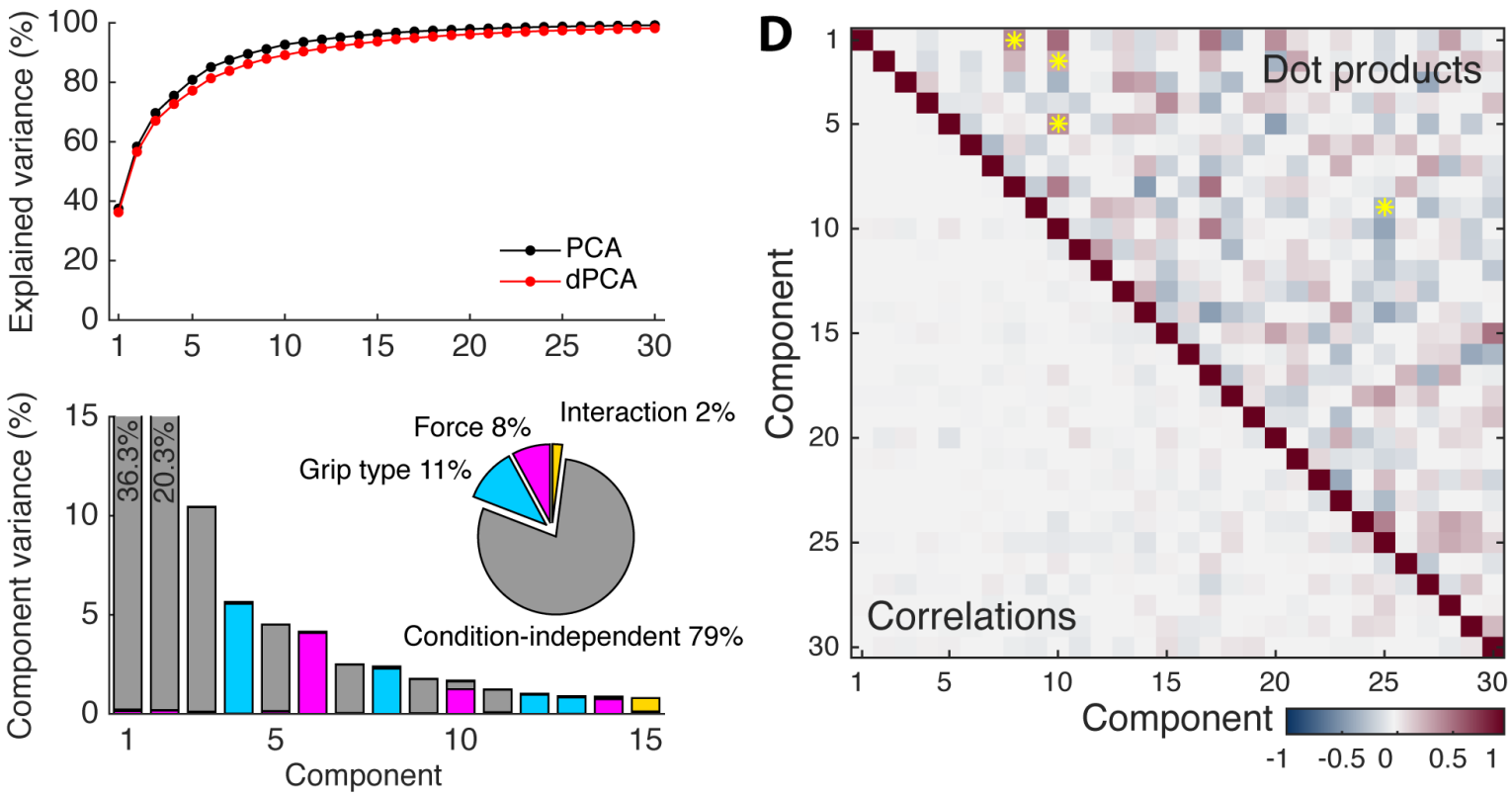
Figure 22. Demixed principal component analysis of F5 in monkey B.

Same as Figure 20, but for the F5 best-channel set of monkey B. 
A

F5 monkey S

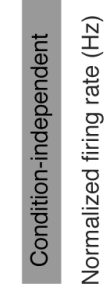
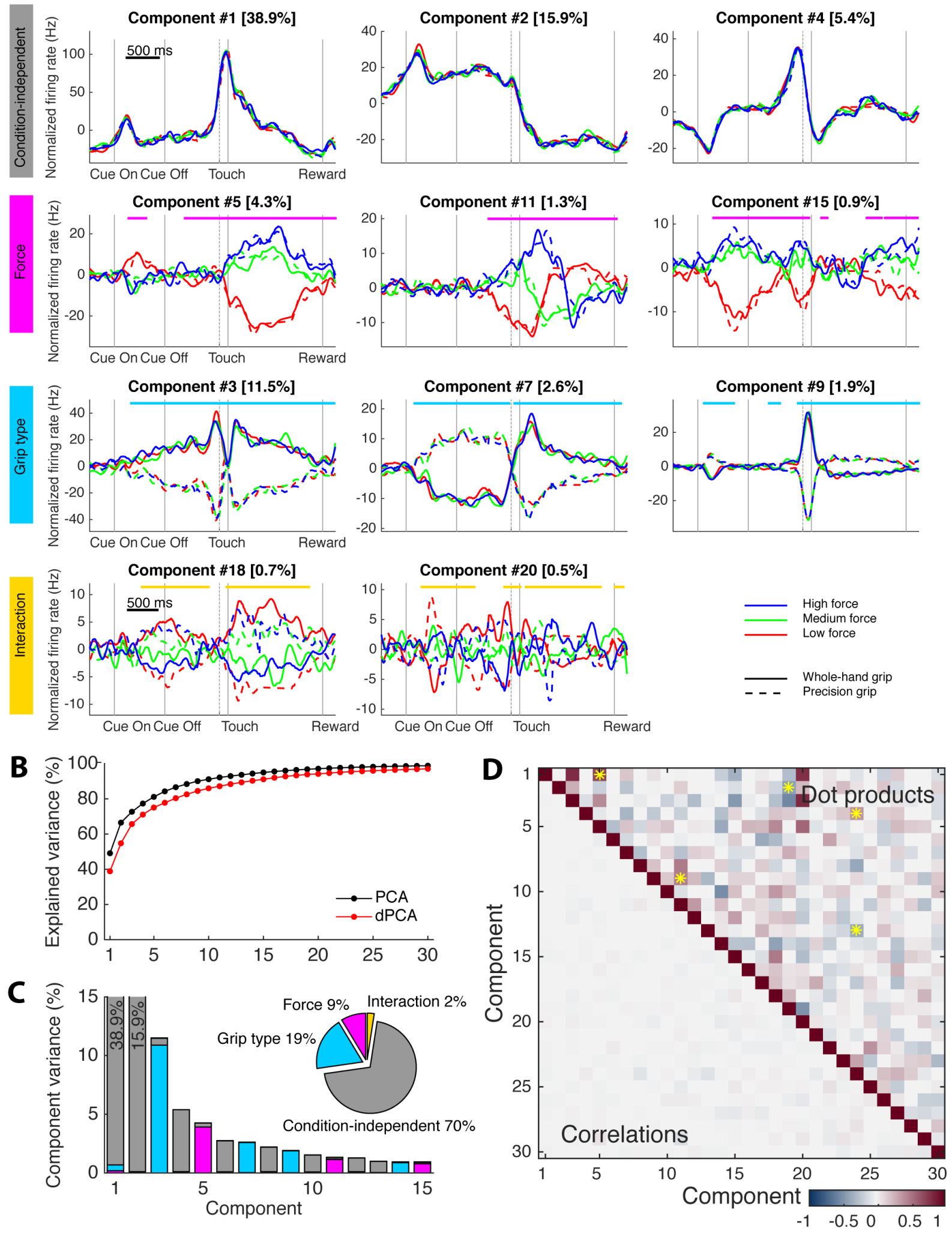
Figure 23. Demixed principal component analysis of F5 in monkey S.

Same as Figure 20, but for the F5 best-channel set of monkey S.

\subsubsection{Demixed components and explained variance in M1}

When we look at Figure 24, the first thing to notice is the low amount of variance explained by the force conditions. This may seem somewhat counter-intuitive, since M1 is known to strongly encode grip force signals (Smith et al., 1975; Hepp-Reymond et al., 1978; HeppReymond and Diener, 1983; Muir and Lemon, 1983; Maier et al., 1993; Hepp-Reymond et al., 1999; Carmena et al., 2003; Hendrix et al., 2009; Milekovic et al., 2015). However, as mentioned before, a low percentage of the total variance does not automatically mean a low amount of information compared to the other areas, as it may be that M1 variance is of a higher magnitude than what is found in F5 and AIP. Nevertheless, it does mean that force variance is low compared to the condition-independent variance and grip type variance.

The components revealed by dPCA in M1 show similar patterns as in AIP and F5, but with much weaker decoding results before movement onset. This matches well with the view of AIP and F5 being more involved with movement planning and M1 being more involved with movement output. 
M1 monkey B
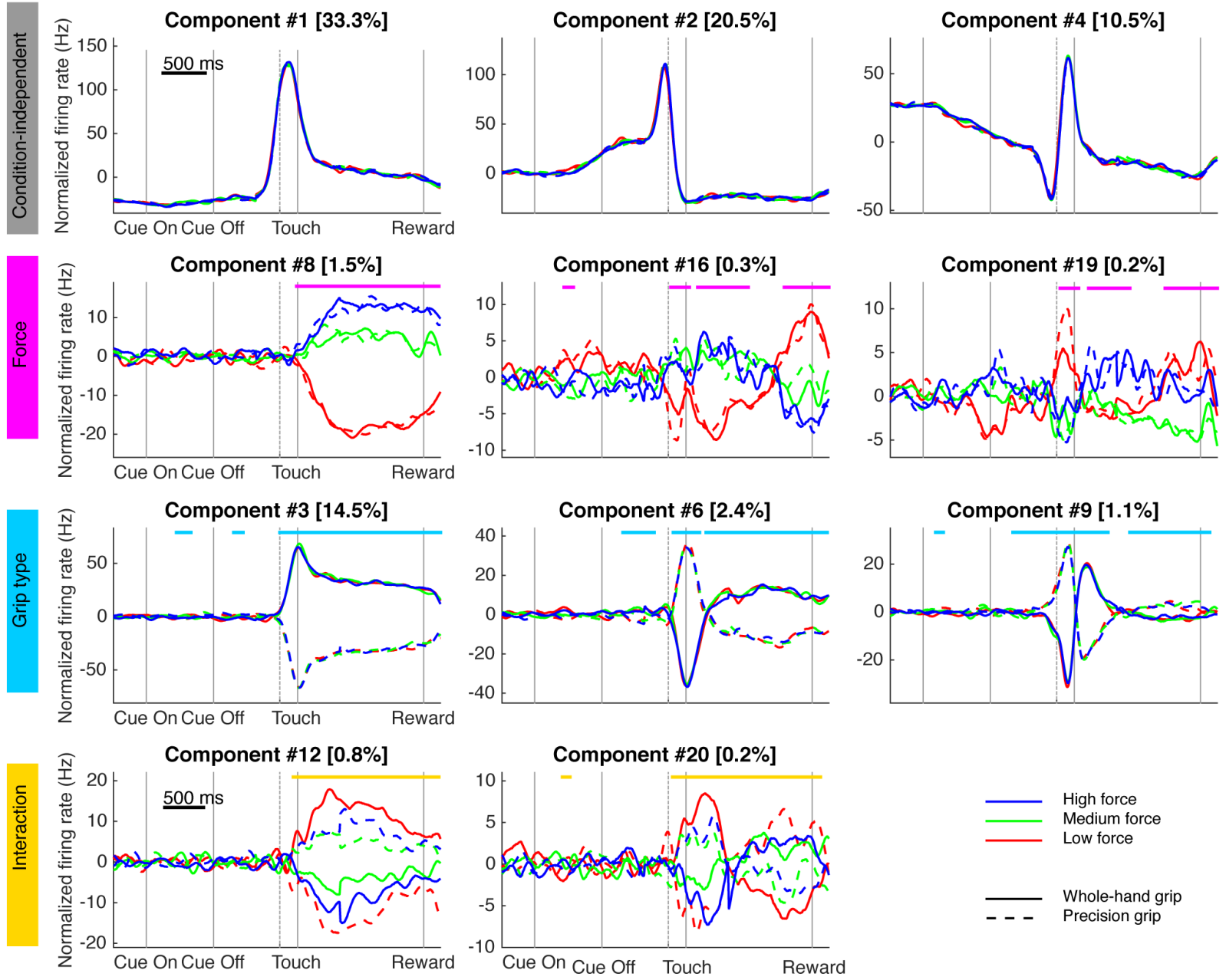

B

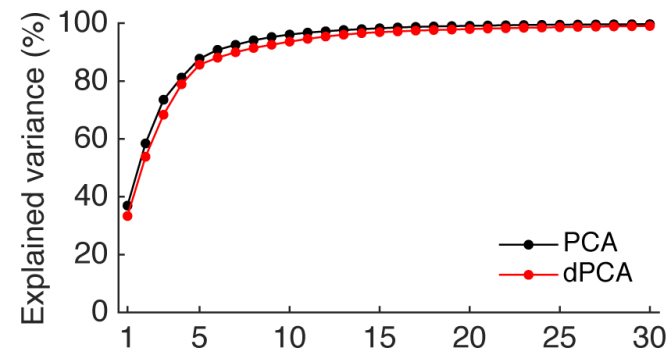

C

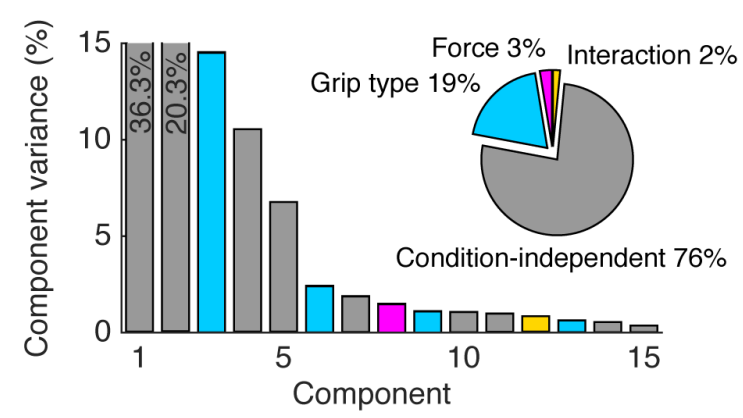

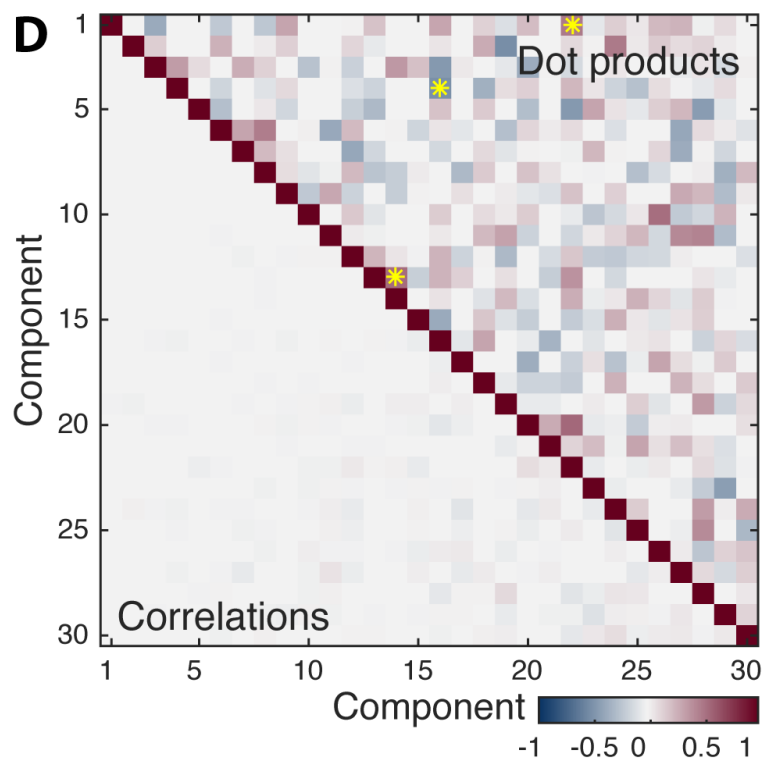


Figure 24. Demixed principal component analysis of M1 in monkey B.

Same as Figure 20, but for the M1 best-channel set of monkey B.

\subsubsection{Response of demixed components to medium- and high force}

In all dPCA component figures (20-24) we see that medium force firing rate is more similar to high force firing rate and that they typically do not diverge until about $200 \mathrm{~ms}$ after Touch. This is in line with the force profiles (Figures 8-10), EMG signals (Figure 11), example neuron's PSTHs (Figure 12), and the medium force modulation index (Figure 17). 


\section{Discussion}

This chapter will summarize the findings of the previous chapter and compare them to what has been found in the literature. I will address concerns that readers might have and argue how our findings contribute to the existing literature.

In this chapter, I:

- Summarize our major findings (Chapter 4.1.)

- Compare the neural coding of grasp force in M1 to the literature (Chapter 4.2.)

- Describe new findings about how F5 codes grasp force control and planning (Chapter 4.3.)

- Describe the neural coding of grasp force control in AIP and discuss its potential role grasp force planning (Chapter 4.4.)

- Discuss how grasp force coding relates to grip type (Chapter 4.5.)

- Bring up alternative explanations for what grasp force coding might represent (Chapter 4.6.)

- Describe some unique aspects of our study (Chapter 4.7.)

- Discuss the functional role of AIP, F5, and M1 in the control of grasp force (Chapter 4.8.)

- Conclude the relevance of our findings (Chapter 4.9.) 


\subsection{Summary}

The goal of this study was to investigate how macaque brain areas AIP, F5, and M1 are involved in the planning and execution of grasp force. We found strong coding of grasp force in units of all three areas, thereby showing for the first time single neuron modulation to grasp force in area AIP. All areas had strongest force modulation during grasp execution and holding. Grasp force planning activity was strongest in F5. M1 units showed some response to the force cue, but hardly any force modulation in the consecutive Memory epoch. Force planning activity in AIP differed between the two investigated monkeys. In monkey $\mathrm{S}$ it was about as strong as in F5, whereas in monkey B it was about as weak as in M1. There was a great variety among units which condition evoked the highest firing rate, the only notable pattern was that the firing rate for the medium force condition was usually between the other conditions or similar to the high force condition. Furthermore, which condition evoked highest firing rate often changed over the course of a trial. Grip type tuning was stronger than grasp force tuning, except for the holding epoch of F5. Grip type and force tuning often interacted, but only in a minority of units did the grip type affect which force condition evoked the highest firing rate.

By using demixed principal component analysis (dPCA), we found that grip type explained more neural variance in the investigated areas than grasp force. In F5 these values were much closer to each other than in the other areas, suggesting a possibly more important role for F5 in controlling grasp force. We also found that force signals could be decoded from all three brain areas during grasp execution and holding. Grasp force decoding before movement onset was uncommon in demixed principal components of AIP and M1 of 
monkey B, but common in AIP of monkey S and in F5 of both monkeys. Altogether, this study showed that F5 encodes grasp force planning strongest among the three investigated areas. Grasp force planning in M1 is quite weak and for AIP this remains unclear due to inconsistency between animals.

\subsection{Grasp force coding in M1}

We found that the percentages of units coding grasp force in M1 reached 40\% during the Hold epoch. This confirms that many M1 units code grasp force, as has been shown repeatedly in the last decades (Smith et al., 1975; Hepp-Reymond et al., 1978; HeppReymond and Diener, 1983; Maier et al., 1993; Hepp-Reymond et al., 1999). We also found some units that modulated their firing rate in relation to the force cue presented, and with dPCA we found one principal component where grasp force could be decoded from a fraction of the Cue epoch and not during the Memory epoch. On the other hand, grasp force was reliably decoded from the Hold epoch in all force components. These findings correspond well to a study which showed that grip force can be decoded from M1 LFP activity during movement and, to a minor extent, also during planning (Milekovic et al., 2015).

dPCA also revealed that the amount of variance explained by grasp force was $3 \%$. This may seem like a low number, but is actually within the range of how much variance a task condition explains in in vivo neurophysiological studies (Kobak et al., 2016). However, it is low compared to variance explained by grip type (19\%). This difference corresponds to the higher percentage of grip type tuned units found in M1. Most of the variance was explained 
by condition-independent components (76\%). As can be seen in Figure 24A, the three largest condition-independent components had peak firing rate around movement onset, suggesting a high contribution of the reaching movement on activity of the hand field of M1.

The much lower contribution of M1 variance to grasp force than to hand shape or reaching movements may seem in conflict with the idea that M1 activity is more concerned with muscle output than with changes in kinematics (Evarts, 1968; Cheney and Fetz, 1980; Hamel-Pâquet et al., 2006). However, it is important to note that muscle activity is affected much more by the reaching movement and grip type than by the force conditions, as can be seen in the EMG signals (Figure 11). It is also interesting to note that demixed component 1 in Figure 24A, which explains 33.3\% of the variance in M1, looks very similar to the EMG responses.

Although the M1 results of this study do not add much new information to the existing literature about grasp force by themselves, it is useful to compare the percentages of tuned units and explained variance from this well investigated area to that of the less studied areas F5 and AIP.

\subsection{Grasp force coding in F5}

It was already shown in the 1990s that neurons of the ventral premotor cortex code grip force (Hepp-Reymond et al., 1994), but it was also said that percentages of force tuned neurons were lower in PMv than in M1. Our results do not fully agree with this, since we 
found that, like M1, about 40\% of units in F5 are tuned for force in the Hold epoch. Before movement onset, we even observed higher percentages of force tuned units in F5 (10-30\%) than in M1 $(<10 \%)$. However, these differences could well be due to different task designs. In the study of Hepp-Reymond and colleagues, monkeys already held the force transducer at the start of a trial, so there was no reaching component. This paradigm is likely most similar to the later part of the Hold epoch in our task design. Indeed, when we look at the last $500 \mathrm{~ms}$ before reward onset (Figure 15 and 16), the percentage of force tuned units is about $10 \%$ lower in F5 than in M1. This could mean that F5 is the major area to plan grasp force and apply the right amount, but when this is achieved, M1 becomes predominant to control static force. More research is required to test this hypothesis.

We were able to predict the grasp force condition in both monkeys from the firing rate of F5 units before movement onset, which was not possible from M1 activity. Even though we did not explicitly test decoding performances in this study, and therefore cannot say which area would be better in predicting grasp force during movement execution, it is likely that both of these areas are suitable targets for decoding grasp force, as was also found in a pilot study for predicting maximum applied grip force (Bataineh et al., 2016).

Interestingly, the percentage of variance explained by the force conditions was much higher in F5 (8\% and 9\% for monkey B and S, respectively) than in M1 (3\% for monkey B), while the grip type variance was lower (11\% in monkey B's F5) or similar (19\% in monkey $S^{\prime}$ F5) to M1 (19\%). Even though this does not reveal which brain area holds more 
information about grasp force, it shows that F5 is affected more by grasp force conditions than $\mathrm{M} 1$ relative to how both areas are affected by grip type.

\subsection{Grasp force coding in AIP}

\subsubsection{Grasp force control}

We have shown, to our knowledge for the first time, single neuron coding for grasp force in AIP. Even though the two animals showed different amounts of grasp force coding in the period before movement onset, a similar proportion of units was modulated by grasp force during movement.

This finding contradicts the idea that the parietal cortex strictly codes kinematics and not kinetics (Kalaska et al., 1990; Hamel-Pâquet et al., 2006), but is in line with other studies that have shown force modulation in the parietal cortex (Ehrsson et al., 2003; Keisker et al., 2010; Neely et al., 2013; Ferrari-Toniolo et al., 2015). Although finding some force modulation in AIP is probably not surprising, we found that $20-30 \%$ of the recorded units was tuned for force in the Hold epoch (Figure 13). In comparison to the percentage of force tuned units M1 (40\%) this is lower, but still substantial.

Another interesting comparison is with grip type tuning. Ever since Taira and colleagues identified grasp-related properties of AIP neurons (Taira et al., 1990), grip type and object shape have been the main features encoded in AIP (for review, see Rizzolatti and Luppino, 2001; Janssen and Scherberger, 2015). Nevertheless, we found that the proportion of AIP 
units modulated by force conditions during Hold was only slightly lower than those modulated by grip type (30-50\%), indicating that grasp force is strongly encoded in AIP.

We found that $5 \%$ and $7 \%$ of the variance was explained by the force conditions in the AIP units of monkey B and S, respectively. These values are similar to what was found M1 and F5, but much lower than what is explained by grip type (18\% in monkey B and $30 \%$, in monkey S). We can conclude from this that the force conditions explain a significant amount of the neural variance, but that grip type conditions are clearly encoded stronger in AIP.

\subsubsection{Grasp force planning}

The role of AIP in the planning of grasp force remains unclear. In monkey B we found very low percentages of AIP units tuned for grasp force and we were not able to decode grasp force before movement onset, very similar to what was found in M1. Conversely, AIP units recorded from monkey S showed high percentages of units tuned for grasp force in the Cue and Memory epoch and the force conditions could be predicted from these epochs, very similar to F5.

Several factors could be responsible for these differences. First of all, it is possible that recordings were not made from exactly the same location. Anatomical studies have identified AIP as a very small sector (5-6 $\mathrm{mm}$ ) in the lateral bank of the anterior intraparietal sulcus (Lewis and Van Essen, 2000), and since the floating microelectrode arrays (FMAs; MicroProbe for Life Science) used in this study are $4 \mathrm{~mm}$ in length, some 
electrodes will record activity just outside of area AIP. Small variations in array placement could therefore affect the results. However, this would probably only slightly change the proportions of types of units found and not create the big differences as observed here.

Another, more likely influence can be the hemisphere from which we recorded, since we recorded signals from monkey B's right hemisphere and monkey S' left hemisphere. Interestingly, several studies have described a difference between left- and right-sided AIP in right-handed humans. Only right-sided AIP activity scaled with imagined grip force (Mizuguchi et al., 2014), increased when subjects were to coordinate gripping and lifting (Ehrsson et al., 2003), and was higher during static grips than during dynamic grips (Keisker et al., 2010; Neely et al., 2013). This suggests that right-sided AIP is more involved in static force control than left-sided AIP. However, grip force scaling could only be impaired by inactivating left-sided AIP in the period before an object is grasped, but not by inactivating right-sided AIP, independent of the hand used by the subjects (Davare et al., 2007). This suggests that left-sided AIP is more involved in planning grasp force.

If these hemispheric differences also exist in monkeys, it could explain why AIP of monkey B (right hemisphere) had weak planning activity, whereas AIP of monkey S (left hemisphere) had strong planning activity. Bilateral recordings from the same animal would be needed to test for differences between right- and left-sided AIP in grasp force planning.

A third possibility for the differences is that the animals used a different strategy to solve the task. We observed in the force profiles of monkey B (Figure 8 and 9) that he did not 
plan differently for the medium- or high force condition. Monkey S seemed to behave similarly (Figure 10), but we found a significant difference in the amount of force applied in the first 100 ms after Touch between the PMF and PHF conditions. This suggests that monkey S understood the cues better, which could correspond to AIP showing more planning activity. However, what speaks against this, is that monkey B did clearly distinguish between the low and other force conditions, just as well as monkey S did. Furthermore, grasp force planning activity was clearly present in area F5 of monkey B. The percentage of force tuned units was slightly lower in F5 for monkey B than for monkey S and this small difference could be related to the different behavior, but the small behavioral differences are unlikely the cause for the large differences between the AIP responses of both monkeys.

\subsection{The relation between grip type and force}

An interesting aspect of our study is that we investigated the neural response to grasp force for two different grip types. The neural responses to these grip types are similar to what has been described in previous studies (Rizzolatti et al., 1988; Taira et al., 1990; Baumann et al., 2009; Fluet et al., 2010; Townsend et al., 2011; Lehmann and Scherberger, 2013; Lehmann and Scherberger, 2015; Menz et al., 2015; Schaffelhofer et al., 2015; Vargas-Irwin et al., 2015; for review, see Rizzolatti and Luppino, 2001; Janssen and Scherberger, 2015;), suggesting a general finding. But how do grip type and force interact? 


\subsubsection{Interaction}

We discussed already how percentages of force tuned units or variance explained by force compare to the similar measures for grip type. But we have also seen that there is often an interaction between these two parameters. In figures 13-16 we can see that when there is force tuning there is also a smaller percentage of interaction tuning. In Figure 14 we showed that interaction tuning often coincided with force tuning, which meant that there was a different response to the force conditions for each grip type, but force coding was not reversed for the grip types. In this case, grip type and force tuning information can be extracted independently. In other cases, it occurred that force tuning was reversed for the other grip type and this was best identified by the moments the grip force and interaction tuning did not coincide, as can be seen in figures $12 \mathrm{~A}$ and $12 \mathrm{D}$. In such cases, the modulation to force conditions is obscured by the effect of grip type and information about the force condition cannot be extracted separately from grip type information. We can see in figures 20-24 that this was the case for $2-3 \%$ of the variance (variance explained by interaction).

Interaction between grip type and force can have different meanings. 1) It could, for example, be related to different muscles that are activated for the two grip types, like we can see in the EMG signals (Figure 11). This might well explain the high percentage of interaction tuned units during Hold in M1, as we know that many M1 neurons fire in close relation to muscle activity (Evarts, 1968; Cheney and Fetz, 1980). But also pre-movement activity, as we observed mainly in F5, could be related to a motor plan of the upcoming muscle activations. 
2) It could also be representing effort or motivation, since we know that decision variables are represented in parietal and frontal areas (for review, see McCoy and Platt, 2005; Andersen and Cui, 2009). We can see in Figure 6 that, although both monkeys had little difficulty performing the task, there was a great difference in motivation among conditions and there was an interaction between grip type and force. Monkey B had a similar motivation for all whole-hand grip conditions, but force affected the motivation for precision grip conditions. For monkey S this was exactly the opposite, as force affected the motivation for whole-hand grip conditions. A motivation coding neuron would therefore show a response to force only for one grip type, which would result in significant interaction tuning.

3) A third possibility is that an interaction response is related to reaction time, as it has been shown recently that reaction time in a reach and grasp task can be predicted from activity in AIP and F5 (Michaels et al., 2015). Figure 7 shows that reaction time depended on both grip type and force, with an interaction between the two parameters. This coupling could potentially also affect the spiking activity of AIP and F5 in the period leading up to movement.

The observed interaction responses are probably caused by a combination of several factors, including the three possibilities just described. These responses illustrate the complexity of how activity of multi- and single units in the cortex relate to grasping movements. However, it is important to note that in only a small percentage of variance the 
information for grip type and force could not be extracted separately, as also found in a similar study of dorsal PMC and M1 (Hendrix et al., 2009).

\subsubsection{Influence of grip types on tuning percentages}

An important question to ask in relation to our results is: does using two grip types increase the amount of force tuned units found? As we discussed before, there are cases when force tuning was only present in one grip type (case 1) and cases when force tuning was present in both grip types, but in reversed order (case 3). If the first case is more dominant, using multiple grip types would result in finding higher percentages of force tuned units, while it would result in lower percentages when case 3 was more dominant. In Figure 14 we can see that interaction tuning occurred together with force tuning (case 1: force tuning is present for only one grip type or case 2 : force tuning is more dominant in one grip type) and without force tuning (case 3: force tuning is reversed in the other grip type) in roughly equal amounts. Only during Hold in Figure 14A and C do we see a simultaneous increase in interaction and force tuning (case 1 or case 2). We can conclude from this that there is no clear trend to which category most recorded units belong. We therefore predict that the percentage of force tuned units would be very similar to what is shown here, if this study was performed with one or more than two grip types. 


\subsubsection{Temporal difference between grip type and force coding}

We found a temporal difference between the onset of grip type, force, and interaction tuning (figures 13-16). Grip type tuning appeared soon after cue onset, while force tuning lagged behind, and interaction tuning lagged farther behind.

An obvious explanation for the earlier rise in grip type tuning is how the two parameters were cued. Grip type was indicated by the location of the cue, while force was indicated by the height of a square within this cue (Figure 3), therefore the monkey must first be aware of the location of the cue, before it can direct its attention to the height of the square.

However, there is reason to believe that even when we used different kinds of cues, we would have observed the same pattern. When grip type and force cues were not presented simultaneously, it was found that grip type could be decoded quite accurately from the delay period before force information was given, while force decoding was much worse when grip type information was not yet given (Milekovic et al., 2015). This possibly could have been because force could not be planned well in advance when grip type was not yet known.

The later rise in interaction tuning in the Cue epoch suggests that grip type and force information are first identified separately, before information is combined to create a mixed representation. 
In area AIP (Figure 13) we can also observe an earlier rise in grip type tuning percentages than force tuning percentages around movement onset. This is interesting since it has been shown in humans that hand shaping is affected by virtually lesioning AIP 270-220 ms before object contact, while grip force scaling is affected by inducing the inactivation 170$120 \mathrm{~ms}$ before object contact (Davare et al., 2007). It is therefore possible that a relation exists between the temporal order when tuning for a certain parameter increases and the optimal timing of virtual lesions for having a behavioral effect on this parameter.

\subsection{Other explanations for observed responses}

We are aware that neural modulation to particular task parameters can always be influenced by confounding factors. We discuss here the influence of muscle activity before the Go signal and that of sensory stimuli.

\subsubsection{Muscle activity before Go signal}

Like other studies using a delayed grasping paradigm (Taira et al., 1990; Baumann et al., 2009; Fluet et al., 2010; Townsend et al., 2011; Lehmann and Scherberger, 2013; Lehmann and Scherberger, 2015; Menz et al., 2015; Schaffelhofer et al., 2015; Vargas-Irwin et al., 2015; for review, see Rizzolatti and Luppino, 2001; Janssen and Scherberger, 2015;), we trained the monkeys to withhold movement, and we did not observe any overt arm movements before the Go signal was given. We therefore assume that pre-movement neural modulation is related to movement planning. However, in Figure 11B we see a significant difference in EDC muscle activity between grip types in the period before 
movement onset. On one day (session B150225) we even noticed a significant difference between force conditions before Movement initiation (i.e. when the hand left the handrest button). Could the pre-movement neural modulation therefore potentially be related to EDC muscle activation?

Although we cannot rule out this possibility, we do have to be aware that the differences in muscle activity between the conditions before movement onset are much smaller than after movement onset, which was much less the case for the neural responses (Figure 12A and B). Furthermore, the response to force conditions was only observed in session B150225, which had very clear EDC signals compared to other muscle recordings, indicating that this was not a general finding. We therefore argue that it is more likely that pre-movement neural modulation is related to planning an upcoming movement than to the small modulation of activity in the EDC muscle, that was significant for grasp force in only one session.

\subsubsection{Visual and haptic representation}

Because our cues about the force conditions were given visually and because F5 and especially AIP are known to be highly responsive to visual information (Janssen and Scherberger, 2015), one could argue that the pre-movement neural modulation we observed merely reflects a response to a visual stimulus and not to the monkey's movement plan. If this were the case, we would expect that the differences in modulation between the low- and medium force conditions is of the same magnitude as the difference between medium- and high force. However, this was clearly not the case. Pre-movement 
neural responses to the medium force of both monkeys, and especially of monkey B, were almost identical to the high force condition and often quite different from low force. As we argued before, since the behavioral data suggests that monkey B did not distinguish between the medium- and high force cues in the Cue epoch, these findings likely reflect the monkey's task understanding.

What is harder to argue against, is that the neural modulation observed after Touch is reflecting the visual feedback we provided or haptic feedback from the grip, since the posterior parietal cortex also receives strong input from somatosensory cortices (Cavada and Goldman-Rakic, 1989b). Although any modulation that already started before or around Touch is likely related to movement representation, some modulation observed in the Hold epoch could be influenced by sensory feedback (i.e. visual or haptic).

Motor and premotor areas are known to be strongly involved in planning and executing movements, making it therefore more likely that the responses to the different force conditions are related to driving grasp force. The parietal cortex, on the other hand, is believed to be an important control center that integrates sensory information and efference copies. It is therefore very likely that sensory feedback responses will also be found in this area.

Nevertheless, a recent study has shown that more PF/PFG neurons were tuned when monkeys used an isometric tool to bring a cursor to a target on a screen, than when animals only observed the cursor moving to the target (Ferrari-Toniolo et al., 2015). Furthermore, 
when the monkeys were exposed to a new condition that required more force to move the cursor for the same distance, the neural firing rate of many parietal neurons changed according to the increased amount of applied force, even though cursor movements were identical to the previous condition. Future analyses can hopefully dissociate what percentage of neural modulation drives the applied force, and what percentage provides feedback about the amount of applied force.

\subsection{Unique aspects of our study}

To better understand the significance of our findings, we address here the unique aspects of our task design and behavior of the monkeys.

\subsubsection{The force range}

We based our force range of 0-12 $\mathrm{N}$ on the amount of force with which the monkeys grasped the handle during training. Previous studies investigating the neural coding of grasp force in macaque monkeys usually did not look at forces higher than $3 \mathrm{~N}$ (Smith et al., 1975; Hepp-Reymond et al., 1978; Hepp-Reymond and Diener, 1983; Muir and Lemon, 1983; Maier et al., 1993; Bennett and Lemon, 1994; Hepp-Reymond et al., 1994; Brochier et al., 1999; Hepp-Reymond et al., 1999; Boudreau et al., 2001). This is remarkable since our monkeys applied around 2-4 $\mathrm{N}$ in the low force condition, i.e. when they only needed to touch the handle and did not need to apply any more force. 
It is possible that our monkeys applied more force because of their training history or because they simply were stronger. Indeed, our monkeys were relatively heavy rhesus macaques (10.1 and $13.5 \mathrm{~kg}$ ) and many of the former studies used the much lighter longtailed macaques (Macaca fascicularis) in their experiments (Smith et al., 1975; HeppReymond et al., 1978; Hepp-Reymond and Diener, 1983; Hepp-Reymond et al., 1994; Brochier et al., 1999; Hepp-Reymond et al., 1999; Boudreau et al., 2001). However, even when monkeys of 2.5-3.5 kg were free to choose their precision grip force to lift a resistive force of $0.3,0.6$, or $1.0 \mathrm{~N}$, they typically applied more than $2 \mathrm{~N}$ for all resistive forces (Boudreau et al., 2001). This suggests that values from 0 to $1 \mathrm{~N}$, which has been suggested as the best range to find correlations between force and neural activity (Hepp-Reymond et al., 1978), is actually below the normal precision grip force of macaque monkeys. One could therefore argue that in these previous studies monkeys were trained to apply their natural grip force or less, while we trained monkeys to apply their natural grip force or more.

Although we know that neural modulations adapt to the range of a force task (HeppReymond et al., 1999), there is reason to believe that investigating a high force range provides different results than a low force range. The blood oxygen level-dependent (BOLD) signal that is measured with fMRI is known to increase in extent and magnitude when subjects apply more force (Thickbroom et al., 1998) and therefore most fMRI studies require subjects to apply at least 15\% of their maximal voluntary contraction (Keisker et al., 2010; Neely et al., 2013). This increased activity of grasp-related brain areas could therefore change the percentages of tuned units observed in our study. 


\subsubsection{Medium- and high force representation}

By analyzing the amount of applied force in the first $100 \mathrm{~ms}$ after Touch, we observed that monkey B did not distinguish between the medium- and high force cue in the Cue epoch, but did distinguish the low force from the other conditions. Also, when monkey B was trained on a task where the force condition was no longer presented again in the hold phase, he simply stayed for 1 second in the medium force range before moving to the high force range in high force trials. Monkey S likely did make a distinction between the medium- and high force cues, but still responded very similar to these conditions in the first $100 \mathrm{~ms}$ of the Hold epoch, which was not the case for low force trials.

Even though the initial training goal was to teach the monkeys to understand the cues from the Cue epoch, it is interesting to see how this apparent task understanding influenced the results. In the PSTHs of the example neurons of Figure 12 we see that the neural signal for medium- and high force trials are almost identical until a few hundred milliseconds after Touch. Only neurons of Monkey S (Figure 12A and B) show some minor difference between medium- and high force before this point. These observations were true for all PSTHs analyzed, which included nearly all units used in this study (data not shown), and for dPCA components (figures 20-24). Although we cannot rule out other causes for these patterns, we argue that they are mainly a reflection of the monkey's task understanding.

\subsection{Functional role of AIP, F5, and M1 in grasp force control}

Knowing that neurons in areas AIP, F5, and M1 modulate activity in relation to grasp force conditions is important for understanding how the brain plans and controls grasping 
movements. However, these results by themselves do not prove an involvement of these areas in the planning and control of grasp force. As just mentioned, other factors, like visual or haptic feedback, could partly also explain the observed task-related modulation.

A common method to test a functional relation between a trait and a brain area is by inactivating that area and testing the behavioral effects. By doing so, a strong consensus has arisen that areas AIP, F5, and M1 are involved in the formation of hand shapes (Gallese et al., 1994; Kubota, 1996; Schieber and Poliakov, 1998; Brochier et al., 1999; Fogassi et al., 2001). Since grasp force is unequivocally linked to hand shape, the amount of force applied onto an object will therefore automatically be affected by a lesion that affects hand shaping, but this does not proof that the inactivated area is really involved in the planning and control of grasp force. Another difficulty with showing the effect of a lesion on grasp force is that monkeys rapidly stop performing a grasping task when they experience a lack of hand control and muscle weakness. It is unclear if the motivation to grasp is lost due to the inability to correctly shape the hand or to produce enough grasp force. Although it has been shown that a monkey was no longer able to maintain a grip for 2 seconds after M1 inactivation, while it was still able to correctly place the thumb and index finger on a manipulandum, no significant decrease in stationary grip force was measured in the successful trials performed after muscimol injection in M1 (Brochier et al., 1999).

Stronger evidence for the involvement of cortical areas in grasp force planning and control, independent from hand shape, comes from a study that used transcranial magnetic stimulation to induce virtual lesions (Davare et al., 2007). The researchers were able to 
show that by inactivating AIP for a specific time frame, grip force scaling could be disturbed, while hand shaping was unaffected. These results suggest that grasp force planning occurs in AIP independent from grip type planning. Even though this temporal dissociation of grip type and force planning may be unique for AIP, it is possible that parallel processing of these two parameters could also occur in F5 and M1, although this may be very hard to disentangle. Altogether we can conclude that the neural modulations we observed are likely related to the functional control of grasp force, but more research is necessary to clearly dissociate this from controlling hand shape.

\subsection{Conclusion}

We have shown in this thesis a strong representation of grasp force control in macaque areas AIP, F5, and M1, as well as grasp force planning representation in F5 of both monkeys and in AIP of one monkey. These findings are in agreement with previous studies that showed a neural representation of grasp force control in M1 (Smith et al., 1975; HeppReymond et al., 1978; Hepp-Reymond and Diener, 1983; Maier et al., 1993; Hepp-Reymond et al., 1999) and F5 (Hepp-Reymond et al., 1994). Modulation of single AIP neurons to grasp force match well with studies that showed an increased activation of this area with fMRI during grasp force control (Ehrsson et al., 2003; Keisker et al., 2010; Neely et al., 2013) and grip force scaling impairment after inactivating AIP (Davare et al., 2007).

In agreement with previous studies we found that grip type was strongly coded in these areas (Rizzolatti et al., 1988; Taira et al., 1990; Baumann et al., 2009; Fluet et al., 2010; Townsend et al., 2011; Lehmann and Scherberger, 2013; Lehmann and Scherberger, 2015; 
Menz et al., 2015; Schaffelhofer et al., 2015; Vargas-Irwin et al., 2015; for review, see Rizzolatti and Luppino, 2001; Janssen and Scherberger, 2015;). Grip type explained a higher percentage of the variance than grasp force, but grasp force was nevertheless a major contributor to the neural variance, especially in F5. We found that grip type and force coding often interact, but for the most part, information about these two features can be extracted separately.

Because lesions of AIP, F5, and M1 are known to affect both hand shaping and grasp force (Gallese et al., 1994; Kubota, 1996; Schieber and Poliakov, 1998; Brochier et al., 1999; Fogassi et al., 2001), we argue that our findings reflect a functional relationship between the observed neural modulations and grasp force control. 


\section{References}

Aflalo T, Kellis S, Klaes C, Lee B, Shi Y, Pejsa K, Shanfield K, Hayes-Jackson S, Aisen M, Heck C, Liu C, Andersen RA (2015) Neurophysiology. Decoding motor imagery from the posterior parietal cortex of a tetraplegic human. Science 348:906-910.

Andersen RA, Cui H (2009) Intention, action planning, and decision making in parietalfrontal circuits. Neuron 63:568-583.

Arce-McShane FI, Hatsopoulos NG, Lee J-C, Ross CF, Sessle BJ (2014) Modulation dynamics in the orofacial sensorimotor cortex during motor skill acquisition. Journal of Neuroscience 34:5985-5997.

Barrese JC, Aceros J, Donoghue JP (2016) Scanning electron microscopy of chronically implanted intracortical microelectrode arrays in non-human primates. J Neural Eng 13:026003.

Barrese JC, Rao N, Paroo K, Triebwasser C, Vargas-Irwin C, Franquemont L, Donoghue JP (2013) Failure mode analysis of silicon-based intracortical microelectrode arrays in non-human primates. J Neural Eng 10:066014.

Bataineh M, McNiel D, Choi J, Hessburg J, Francis J (2016) Pilot Study for Grip Force Prediction Using Neural Signals from Different Brain Regions. Southern Biomedical Engineering Conference 2016, pp 19-20.

Baumann MA, Fluet MC, Scherberger H (2009) Context-Specific Grasp Movement Representation in the Macaque Anterior Intraparietal Area. Journal of Neuroscience 29:6436-6448.

Bennett KM, Lemon RN (1994) The influence of single monkey cortico-motoneuronal cells at different levels of activity in target muscles. J Physiol (Lond) 477:291-307.

Binkofski F, Dohle C, Posse S, Stephan KM, Hefter H, Seitz RJ, Freund HJ (1998) Human anterior intraparietal area subserves prehension: a combined lesion and functional MRI activation study. Neurology 50:1253-1259.

Boline J, Ashe J (2005) On the relations between single cell activity in the motor cortex and the direction and magnitude of three-dimensional dynamic isometric force. Experimental Brain Research 167:148-159.

Borra E, Belmalih A, Gerbella M, Rozzi S, Luppino G (2010) Projections of the hand field of the macaque ventral premotor area F5 to the brainstem and spinal cord. J Comp Neurol 518:2570-2591.

Boudreau MJ, Brochier T, Paré M, Smith AM (2001) Activity in ventral and dorsal premotor cortex in response to predictable force-pulse perturbations in a precision grip task. 
Journal of Neurophysiology 86:1067-1078.

Britten KH, Shadlen MN, Newsome WT, Movshon JA (1992) The analysis of visual motion: a comparison of neuronal and psychophysical performance. J Neurosci 12:4745-4765.

Brochier T, Boudreau MJ, Paré M, Smith AM (1999) The effects of muscimol inactivation of small regions of motor and somatosensory cortex on independent finger movements and force control in the precision grip. Experimental Brain Research 128:31-40.

Carmena JM, Lebedev MA, Crist RE, O'Doherty JE, Santucci DM, Dimitrov DF, Patil PG, Henriquez CS, Nicolelis MAL (2003) Learning to control a brain-machine interface for reaching and grasping by primates. PLoS Biol 1:E42.

Cavada C, Goldman-Rakic PS (1989a) Posterior parietal cortex in rhesus monkey: I. Parcellation of areas based on distinctive limbic and sensory corticocortical connections. J Comp Neurol 287:393-421.

Cavada C, Goldman-Rakic PS (1989b) Posterior parietal cortex in rhesus monkey: II. Evidence for segregated corticocortical networks linking sensory and limbic areas with the frontal lobe. J Comp Neurol 287:422-445.

Chen J, Reitzen SD, Kohlenstein JB, Gardner EP (2009) Neural Representation of Hand Kinematics During Prehension in Posterior Parietal Cortex of the Macaque Monkey. Journal of Neurophysiology 102:3310-3328.

Cheney PD, Fetz EE (1980) Functional classes of primate corticomotoneuronal cells and their relation to active force. Journal of Neurophysiology 44:773-791.

Cisek P (2012) Making decisions through a distributed consensus. Current Opinion in Neurobiology 22:927-936.

Cisek P, Kalaska JF (2005) Neural correlates of reaching decisions in dorsal premotor cortex: specification of multiple direction choices and final selection of action. Neuron 45:801-814.

Cui H, Andersen RA (2011) Different representations of potential and selected motor plans by distinct parietal areas. Journal of Neuroscience 31:18130-18136.

Culham JC, Danckert SL, Souza JFXD, Gati JS, Menon RS, Goodale MA (2003) Visually guided grasping produces fMRI activation in dorsal but not ventral stream brain areas. Experimental Brain Research 153:180-189.

Davare M, Andres M, Clerget E, Thonnard JL, Olivier E (2007) Temporal Dissociation between Hand Shaping and Grip Force Scaling in the Anterior Intraparietal Area. J Neurosci 27:3974-3980.

Desimone R, Schein SJ (1987) Visual properties of neurons in area V4 of the macaque: 
sensitivity to stimulus form. Journal of Neurophysiology 57:835-868.

Dickey AS, Suminski A, Amit Y, Hatsopoulos NG (2009) Single-unit stability using chronically implanted multielectrode arrays. Journal of Neurophysiology 102:13311339.

Dubner R, Zeki SM (1971) Response properties and receptive fields of cells in an anatomically defined region of the superior temporal sulcus in the monkey. Brain Res $35: 528-532$.

Ehrsson HH, Fagergren A, Johansson RS, Forssberg H (2003) Evidence for the involvement of the posterior parietal cortex in coordination of fingertip forces for grasp stability in manipulation. Journal of Neurophysiology 90:2978-2986.

Evarts EV (1968) Relation of pyramidal tract activity to force exerted during voluntary movement. Journal of Neurophysiology 31:14-27.

Fattori P, Breveglieri R, Raos V, Bosco A, Galletti C (2012) Vision for Action in the Macaque Medial Posterior Parietal Cortex. Journal of Neuroscience 32:3221-3234.

Felleman DJ, Van Essen DC (1991) Distributed hierarchical processing in the primate cerebral cortex. Cereb Cortex 1:1-47.

Ferraina S, Battaglia-Mayer A, Genovesio A, Archambault P, Caminiti R (2009) Parietal encoding of action in depth. Neuropsychologia 47:1409-1420.

Ferrari-Toniolo S, Papazachariadis O, Visco-Comandini F, Salvati M, D’Elia A, Di Berardino F, Caminiti R, Battaglia-Mayer A (2014) A visuomotor disorder in the absence of movement: does optic ataxia generalize to learned isometric hand action? Neuropsychologia 63:59-71.

Ferrari-Toniolo S, Visco-Comandini F, Papazachariadis O, Caminiti R, Battaglia-Mayer A (2015) Posterior Parietal Cortex Encoding of Dynamic Hand Force Underlying HandObject Interaction. J Neurosci 35:10899-10910.

Flint RD, Wang PT, Wright ZA, King CE, Krucoff MO, Schuele SU, Rosenow JM, Hsu FPK, Liu CY, Lin JJ, Sazgar M, Millett DE, Shaw SJ, Nenadic Z, Do AH, Slutzky MW (2014) Extracting kinetic information from human motor cortical signals. Neuroimage 101:695-703.

Fluet M-C, Baumann MA, Scherberger H (2010) Context-specific grasp movement representation in macaque ventral premotor cortex. Journal of Neuroscience 30:15175-15184.

Fogassi L, Gallese V, Buccino G, Craighero L, Fadiga L, Rizzolatti G (2001) Cortical mechanism for the visual guidance of hand grasping movements in the monkey: A reversible inactivation study. Brain 124:571-586. 
Ford JM, Perez VB, Mathalon DH (2012) Neurophysiology of a possible fundamental deficit in schizophrenia. World Psychiatry 11:58-60.

Freiwald WA, Tsao DY (2010) Functional compartmentalization and viewpoint generalization within the macaque face-processing system. Science 330:845-851.

Fritsch T, Hitzig E (1870) Ueber die elektrische Erregbarkeit des Grosshirns [About the electrical excitability of the cerebral cortex]. Arch. Anat. Physiol.Leipzig 300-332.

Gallese V, Murata A, Kaseda M, Niki N, Sakata H (1994) Deficit of hand preshaping after muscimol injection in monkey parietal cortex. Neuroreport 5:1525-1529.

Georgopoulos AP, Ashe J, Smyrnis N, Taira M (1992) The motor cortex and the coding of force. Science 256:1692-1695.

Goodale MA, Meenan JP, Bülthoff HH, Nicolle DA, Murphy KJ, Racicot CI (1994) Separate neural pathways for the visual analysis of object shape in perception and prehension. Current Biology 4:604-610.

Goodale MA, Milner AD (1992) Separate visual pathways for perception and action. Trends Neurosci 15:20-25.

Gozani SN, Miller JP (1994) Optimal discrimination and classification of neuronal action potential waveforms from multiunit, multichannel recordings using software-based linear filters. IEEE Trans Biomed Eng 41:358-372.

Graziano MSA, Aflalo TNS, Cooke DF (2005) Arm movements evoked by electrical stimulation in the motor cortex of monkeys. Journal of Neurophysiology 94:4209-4223.

Graziano MSA, Taylor CSR, Moore T (2002) Complex movements evoked by microstimulation of precentral cortex. Neuron 34:841-851.

Gupta R, Ashe J (2009) Offline decoding of end-point forces using neural ensembles: application to a brain-machine interface. IEEE Trans Neural Syst Rehabil Eng 17:254262.

Haaxma R, Kuypers HG (1975) Intrahemispheric cortical connexions and visual guidance of hand and finger movements in the rhusus monkey. Brain 98:239-260.

Hamel-Pâquet C, Sergio LE, Kalaska JF (2006) Parietal area 5 activity does not reflect the differential time-course of motor output kinetics during arm-reaching and isometricforce tasks. Journal of Neurophysiology 95:3353-3370.

Hanks TD, Mazurek ME, Kiani R, Hopp E, Shadlen MN (2011) Elapsed Decision Time Affects the Weighting of Prior Probability in a Perceptual Decision Task. Journal of Neuroscience 31:6339-6352. 
Hendrix CM, Mason CR, Ebner TJ (2009) Signaling of grasp dimension and grasp force in dorsal premotor cortex and primary motor cortex neurons during reach to grasp in the monkey. Journal of Neurophysiology 102:132-145.

Hepp-Reymond M, Kirkpatrick-Tanner M, Gabernet L, Qi HX, Weber B (1999) Contextdependent force coding in motor and premotor cortical areas. Experimental Brain Research 128:123-133.

Hepp-Reymond MC, Diener R (1983) Neural coding of force and of rate of force change in the precentral finger region of the monkey. Exp Brain Res 7:315-326.

Hepp-Reymond MC, Hüsler EJ, Maier MA, Ql HX (1994) Force-related neuronal activity in two regions of the primate ventral premotor cortex. Can J Physiol Pharmacol 72:571579.

Hepp-Reymond MC, Wyss UR, Anner R (1978) Neuronal coding of static force in the primate motor cortex. J Physiol (Paris) 74:287-291.

Huang R-S, Chen C-F, Tran AT, Holstein KL, Sereno MI (2012) Mapping multisensory parietal face and body areas in humans. Proc Natl Acad Sci USA 109:18114-18119.

Hubel DH, Wiesel TN (1968) Receptive fields and functional architecture of monkey striate cortex. J Physiol (Lond) 195:215-243.

Janssen P, Scherberger H (2015) Visual guidance in control of grasping. Annu Rev Neurosci 38:69-86.

Kaas JH, Stepniewska I (2016) Evolution of posterior parietal cortex and parietal-frontal networks for specific actions in primates. J Comp Neurol 524:595-608.

Kalaska JF, Cohen DAD, Prud'homme M, Hyde ML (1990) Parietal area 5 neuronal activity encodes movement kinematics, not movement dynamics. Experimental Brain Research 80:351-364.

Keisker B, Hepp-Reymond M-C, Blickenstorfer A, Kollias SS (2010) Differential representation of dynamic and static power grip force in the sensorimotor network. European Journal of Neuroscience 31:1483-1491.

Kennerley SW, Dahmubed AF, Lara AH, Wallis JD (2009) Neurons in the frontal lobe encode the value of multiple decision variables. J Cogn Neurosci 21:1162-1178.

Klaes C, Westendorff S, Chakrabarti S, Gail A (2011) Choosing goals, not rules: deciding among rule-based action plans. Neuron 70:536-548.

Kobak D, Brendel W, Constantinidis C, Feierstein CE, Kepecs A, Mainen ZF, Qi X-L, Romo R, Uchida N, Machens CK (2016) Demixed principal component analysis of neural population data. Elife 5:9424. 
Kraskov A, Dancause N, Quallo MM, Shepherd S, Lemon RN (2009) Corticospinal neurons in macaque ventral premotor cortex with mirror properties: a potential mechanism for action suppression? Neuron 64:922-930.

Kubota K (1996) Motor cortical muscimol injection disrupts forelimb movement in freely moving monkeys. Neuroreport 7:2379-2384.

Lawrence DG, Kuypers HGJM (1968) The functional organization of the motor system in the monkey. Brain 91:1-18.

Lee H, Bellamkonda RV, Sun W, Levenston ME (2005) Biomechanical analysis of silicon microelectrode-induced strain in the brain. J Neural Eng 2:81-89.

Lehky SR, Sereno AB (2007) Comparison of shape encoding in primate dorsal and ventral visual pathways. Journal of Neurophysiology 97:307-319.

Lehmann SJ, Scherberger H (2013) Reach and gaze representations in macaque parietal and premotor grasp areas. Journal of Neuroscience 33:7038-7049.

Lehmann SJ, Scherberger H (2015) Spatial Representations in Local Field Potential Activity of Primate Anterior Intraparietal Cortex (AIP). PLoS ONE 10:e0142679.

Lewis JW, Van Essen DC (2000) Corticocortical connections of visual, sensorimotor, and multimodal processing areas in the parietal lobe of the macaque monkey. J Comp Neurol 428:112-137.

Luppino G, Murata A, Govoni P, Matelli M (1999) Largely segregated parietofrontal connections linking rostral intraparietal cortex (areas AIP and VIP) and the ventral premotor cortex (areas F5 and F4). Experimental Brain Research 128:181-187.

Maier MA, Bennett KM, Hepp-Reymond MC, Lemon RN (1993) Contribution of the monkey corticomotoneuronal system to the control of force in precision grip. Journal of Neurophysiology 69:772-785.

Maris E, Oostenveld R (2007) Nonparametric statistical testing of EEG- and MEG-data. Journal of Neuroscience Methods 164:177-190.

Marzke MW, Marzke RF (2000) Evolution of the human hand: approaches to acquiring, analysing and interpreting the anatomical evidence. J Anat 197 ( Pt 1):121-140.

McCoy AN, Platt ML (2005) Expectations and outcomes: decision-making in the primate brain. J Comp Physiol A Neuroethol Sens Neural Behav Physiol 191:201-211.

Menz VK, Schaffelhofer S, Scherberger H (2015) Representation of continuous hand and arm movements in macaque areas M1, F5, and AIP: a comparative decoding study. J Neural Eng 12:056016.

Michaels JA, Dann B, Intveld RW, Scherberger H (2015) Predicting Reaction Time from the 
Neural State Space of the Premotor and Parietal Grasping Network. J Neurosci 35:11415-11432.

Milekovic T, Truccolo W, Grün S, Riehle A, Brochier T (2015) Local field potentials in primate motor cortex encode grasp kinetic parameters. Neuroimage 114:338-355.

Mizuguchi N, Nakata H, Kanosue K (2014) Activity of right premotor-parietal regions dependent upon imagined force level: an fMRI study. Front Hum Neurosci 8:810.

Mollazadeh M, Aggarwal V, Davidson AG, Law AJ, Thakor NV, Schieber MH (2011) Spatiotemporal Variation of Multiple Neurophysiological Signals in the Primary Motor Cortex during Dexterous Reach-to-Grasp Movements. Journal of Neuroscience 31:15531-15543.

Muir RB, Lemon RN (1983) Corticospinal neurons with a special role in precision grip. Brain Res 261:312-316.

Musial PG, Baker SN, Gerstein GL, King EA, Keating JG (2002) Signal-to-noise ratio improvement in multiple electrode recording. Journal of Neuroscience Methods 115:29-43.

National Research Council (US) (2003) Guidelines for the Care and Use of Mammals in Neuroscience and Behavioral Research. Washington (DC): National Academies Press (US).

Neely KA, Coombes SA, Planetta PJ, Vaillancourt DE (2013) Segregated and overlapping neural circuits exist for the production of static and dynamic precision grip force. Hum Brain Mapp 34:698-712.

Newsome WT, Wurtz RH, Komatsu H (1988) Relation of cortical areas MT and MST to pursuit eye movements. II. Differentiation of retinal from extraretinal inputs. Journal of Neurophysiology 60:604-620.

Ninu A, Dosen S, Muceli S, Rattay F, Dietl H, Farina D (2014) Closed-loop control of grasping with a myoelectric hand prosthesis: which are the relevant feedback variables for force control? IEEE Trans Neural Syst Rehabil Eng 22:1041-1052.

Northcutt RG (2002) Understanding vertebrate brain evolution. Integr Comp Biol 42:743756.

Pardo-Vazquez JL, Leboran V, Acuña C (2008) Neural correlates of decisions and their outcomes in the ventral premotor cortex. Journal of Neuroscience 28:12396-12408.

Pardo-Vazquez JL, Padron I, Fernandez-Rey J, Acuña C (2011) Decision-making in the ventral premotor cortex harbinger of action. Front Integr Neurosci 5:54. 
Penfield W, Rasmussen T (1950) The Cerebral Cortex of Man. A Clinical Study of Localization of Function. New York: Macmillan.

Pesaran B, Nelson MJ, Andersen RA (2008) Free choice activates a decision circuit between frontal and parietal cortex. Nature 453:406-409.

Platt ML, Glimcher PW (1999) Neural correlates of decision variables in parietal cortex. Nature 400:233-238.

Quiroga RQ, Nadasdy Z, Ben-Shaul Y (2004) Unsupervised spike detection and sorting with wavelets and superparamagnetic clustering. Neural computation.

Rathelot J-A, Strick PL (2006) Muscle representation in the macaque motor cortex: an anatomical perspective. Proceedings of the National Academy of Sciences 103:82578262.

Rathelot J-A, Strick PL (2009) Subdivisions of primary motor cortex based on corticomotoneuronal cells. Proc Natl Acad Sci USA 106:918-923.

Rizzolatti G, Camarda R, Fogassi L, Gentilucci M, Luppino G, Matelli M (1988) Functional organization of inferior area 6 in the macaque monkey. Experimental Brain Research 71:491-507.

Rizzolatti G, Luppino G (2001) The cortical motor system. Neuron 31:889-901.

Rose KD (1994) The earliest primates. Evolutionary Anthropology: Issues, News, and Reviews. Jan 1;3(5):159-73.

Sakata H, Taira M, Murata A, Mine S (1995) Neural mechanisms of visual guidance of hand action in the parietal cortex of the monkey. Cereb Cortex 5:429-438.

Schaffelhofer S, Agudelo-Toro A, Scherberger H (2015) Decoding a wide range of hand configurations from macaque motor, premotor, and parietal cortices. Journal of Neuroscience 35:1068-1081.

Scherberger H, Andersen RA (2007) Target selection signals for arm reaching in the posterior parietal cortex. Journal of Neuroscience 27:2001-2012.

Schieber MH, Poliakov AV (1998) Partial inactivation of the primary motor cortex hand area: effects on individuated finger movements. J Neurosci 18:9038-9054.

Seelke AMH, Padberg JJ, Disbrow E, Purnell SM, Recanzone G, Krubitzer L (2012) Topographic Maps within Brodmann's Area 5 of macaque monkeys. Cerebral Cortex 22:1834-1850.

Shimazu H, Maier MA, Cerri G, Kirkwood PA, Lemon RN (2004) Macaque ventral premotor cortex exerts powerful facilitation of motor cortex outputs to upper limb motoneurons. Journal of Neuroscience 24:1200-1211. 
Smith AM, Hepp-Reymond MC, Wyss UR (1975) Relation of activity in precentral cortical neurons to force and rate of force change during isometric contractions of finger muscles. Experimental Brain Research 23:315-332.

So NY, Stuphorn V (2010) Supplementary Eye Field Encodes Option and Action Value for Saccades With Variable Reward. Journal of Neurophysiology 104:2634-2653.

Srivastava S, Orban GA, De Mazière PA, Janssen P (2009) A distinct representation of threedimensional shape in macaque anterior intraparietal area: fast, metric, and coarse. Journal of Neuroscience 29:10613-10626.

Stepniewska I, Gharbawie OA, Burish MJ, Kaas JH (2014) Effects of muscimol inactivations of functional domains in motor, premotor, and posterior parietal cortex on complex movements evoked by electrical stimulation. Journal of Neurophysiology 111:11001119.

Sugrue LP, Corrado GS, Newsome WT (2004) Matching behavior and the representation of value in the parietal cortex. Science 304:1782-1787.

Taira M, Boline J, Smyrnis N, Georgopoulos AP, Ashe J (1996) On the relations between single cell activity in the motor cortex and the direction and magnitude of threedimensional static isometric force. Experimental Brain Research 109:367-376.

Taira M, Mine S, Georgopoulos AP, Murata A, Sakata H (1990) Parietal cortex neurons of the monkey related to the visual guidance of hand movement. Experimental Brain Research 83:29-36.

Thickbroom GW, Phillips BA, Morris I, Byrnes ML, Mastaglia FL (1998) Isometric forcerelated activity in sensorimotor cortex measured with functional MRI. Experimental Brain Research 121:59-64.

Townsend BR, Subasi E, Scherberger H (2011) Grasp movement decoding from premotor and parietal cortex. Journal of Neuroscience 31:14386-14398.

Ungerleider LG, Mishkin M (1982) Two cortical visual systems Ingle DJ, Goodale MA, eds. Analysis of Visual Behavior:549-585.

Vargas-Irwin CE, Franquemont L, Black MJ, Donoghue JP (2015) Linking Objects to Actions: Encoding of Target Object and Grasping Strategy in Primate Ventral Premotor Cortex. Journal of Neuroscience 35:10888-10897.

Vargas-Irwin CE, Shakhnarovich G, Yadollahpour P, Mislow JMK, Black MJ, Donoghue JP (2010) Decoding Complete Reach and Grasp Actions from Local Primary Motor Cortex Populations. Journal of Neuroscience 30:9659-9669.

Watson T (2007) NC3Rs Guidelines. :1-24. 
Wolpert DM, Landy MS (2012) Motor control is decision-making. Current Opinion in Neurobiology 22:996-1003.

Young RW (2003) Evolution of the human hand: the role of throwing and clubbing. J Anat 202:165-174.

Zaepffel M, Trachel R, Kilavik BE, Brochier T (2013) Modulations of EEG beta power during planning and execution of grasping movements. Di Russo F, ed. PLoS ONE 8:e60060. 


\section{Curriculum vitae}

\section{Rijk W. in 't Veld}

Zimmermannstraße 60, 37075 Göttingen, Germany

Tel: +4915737637073 E-mail: rintveld@,dpz.eu

Date of birth: $30^{\text {th }}$ October, 1987 Nationality: Netherlands

\section{PhD Project}
2012-present 'Neural coding of grasp force planning German primate and execution in macaque premotor and center, Göttingen parietal cortex'
Supervisor: Hansjörg Scherberger

\section{Education}

2009-2012 MSc Neuroscience and Cognition 2005-2008 BSc Biology

\section{Internships}

2011

2009-2010
'The contribution of visual and proprioceptive feedback to brainmachine interface control'

Supervisor: Nicholas G. Hatsopoulos

Duration: 8 months
Utrecht University

Utrecht University
'The effect of memory load on spiking activity in the primate prefrontal cortex'
Supervisors: Richard J.A. van Wezel \&
Nick F. Ramsey
Duration: 9 months

University of Chicago

\section{Methods courses}

2013

2010

2010

2009
Multielectrode Array Recordings from the Retina

Experimental Neurophysiology

Laboratory Animal Science

Measuring behaviour
University of Göttingen

VU University

Amsterdam

Utrecht University

Utrecht University 


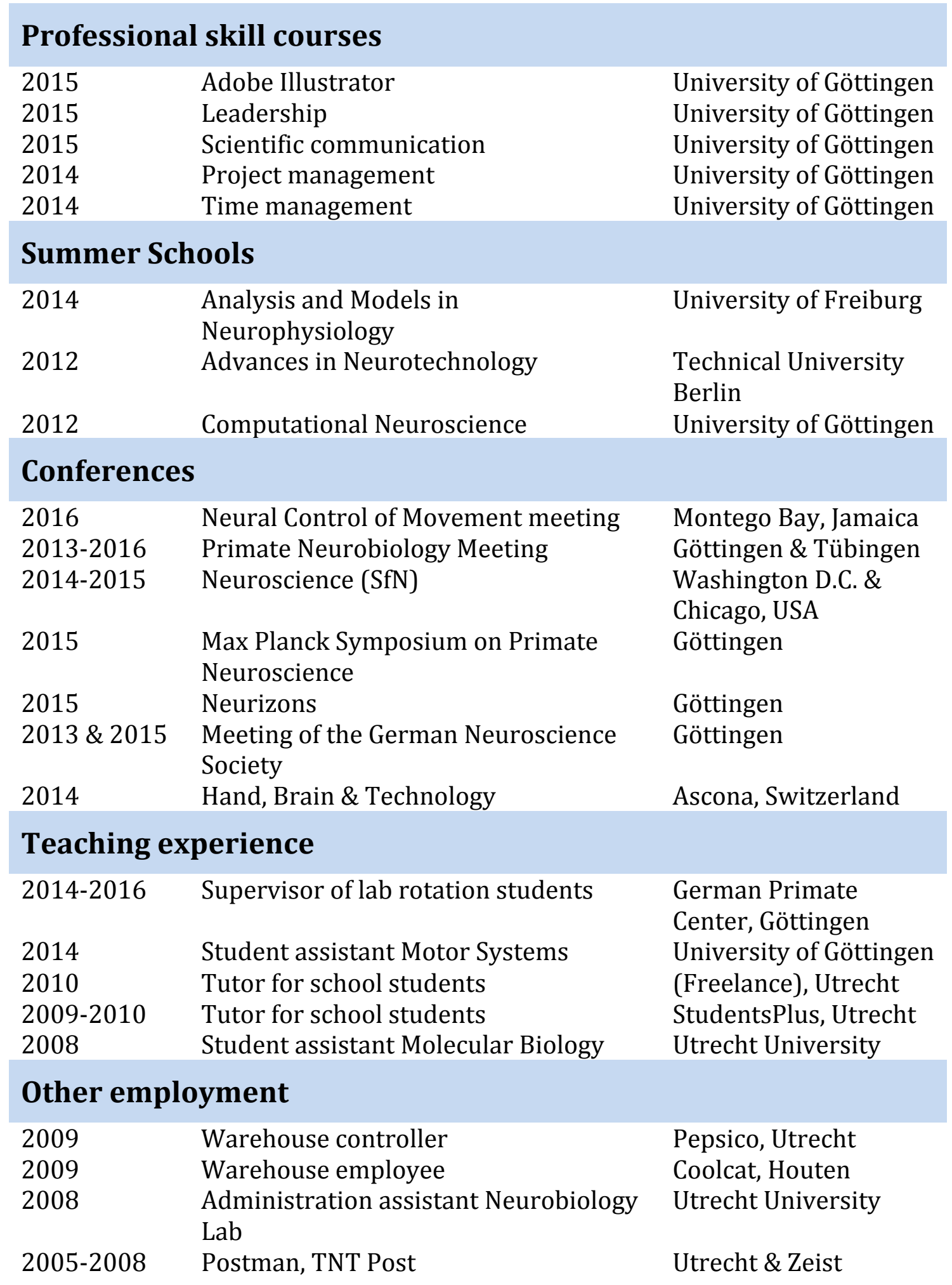




\section{Extracurricular activities}

2015-2016 Student representative of Georg-August University School of

Science (GAUSS)

2014-2016 Student survey coordinator of GAUSS

2014-2015 Student representative of Center for Systems Neuroscience

2009-2010 Financial manager of the Master's student representatives

2009-2010 Webmaster of track \& field association AV Fit Zeist

2009-2010 Student symposium co-organizer, task: Webmaster

\section{Languages}

$\begin{array}{ll}\text { Dutch } & \text { Mother tongue } \\ \text { English } & \text { Fluent } \\ \text { German } & \text { Fluent } \\ \text { French } & \text { Beginner }\end{array}$

\section{Computer skills}

Operating Microsoft Windows and Mac OS

System

Microsoft Word, Excel and Powerpoint

Office

Scientific Matlab, Labview and SPSS

software

Design

Website

Adobe Illustrator

Drupal and Joomla 


\section{Appendix}

In addition to the project described in this thesis, I also assisted in a side project, titled:

'Predicting Reaction Time from the Neural State Space of the Premotor and Parietal

Grasping Network'. This work was published in The Journal of Neuroscience on August $12^{\text {th }}$

2015 (DOI: http://dx.doi.org/10.1523/JNEUROSCl.1714-15.2015). I collaborated in this work with

Jonathan A. Michaels, Benjamin Dann, and Hansjörg Scherberger, by assisting in designing

the study and collecting data. Here follows a reprint of this article. 


\title{
Predicting Reaction Time from the Neural State Space of the Premotor and Parietal Grasping Network
}

\author{
미onathan A. Michaels, ${ }^{1}$ Benjamin Dann, ${ }^{1}$ Rijk W. Intveld, ${ }^{1}$ and ${ }^{\oplus}$ Hansjörg Scherberger ${ }^{1,2}$ \\ ${ }^{1}$ German Primate Center, D-37077 Göttingen, Germany, and ${ }^{2}$ Faculty of Biology, Georg August University Göttingen, D-37073 Göttingen, Germany
}

\begin{abstract}
Neural networks of the brain involved in the planning and execution of grasping movements are not fully understood. The network formed by macaque anterior intraparietal area (AIP) and hand area (F5) of the ventral premotor cortex is implicated strongly in the generation of grasping movements. However, the differential role of each area in this frontoparietal network is unclear. We recorded spiking activity from many electrodes in parallel in AIP and F5 while three macaque monkeys (Macaca mulatta) performed a delayed grasping task. By analyzing neural population activity during action preparation, we found that state space analysis of simultaneously recorded units is significantly more predictive of subsequent reaction times (RTs) than traditional methods. Furthermore, because we observed a wide variety of individual unit characteristics, we developed the sign-corrected average rate (SCAR) method of neural population averaging. The SCAR method was able to explain at least as much variance in RT overall as state space methods. Overall, F5 activity predicted RT (18\% variance explained) significantly better than AIP (6\%). The SCAR methods provides a straightforward interpretation of population activity, although other state space methods could provide richer descriptions of population dynamics. Together, these results lend support to the differential role of the parietal and frontal cortices in preparation for grasping, suggesting that variability in preparatory activity in F5 has a more potent effect on trial-to-trial RT variability than AIP.
\end{abstract}

Key words: grasping; nonhuman primate; parietal; premotor; single unit recording

Significance Statement

Grasping movements are planned before they are executed, but how is the preparatory activity in a population of neurons related to the subsequent reaction time (RT)? A population analysis of the activity of many neurons recorded in parallel in macaque premotor (F5) and parietal (AIP) cortices during a delayed grasping task revealed that preparatory activity in F5 could explain a threefold larger fraction of variability in trial-to-trial RT than AIP. These striking differences lend additional support to a differential role of the parietal and premotor cortices in grasp movement preparation, suggesting that $\mathrm{F} 5$ has a more direct influence on trial-to-trial variability and movement timing, whereas AIP might be more closely linked to overall movement intentions.

\section{Introduction}

In the sport of fencing, rapid actions are required on the millisecond scale. Small rotations of the wrist can make the difference between a hit and a complete miss. The response of athletes to various attacks is highly variable, despite the rigorously trained nature of their skill set. What are the factors that contribute to the variability of such complex actions? It is known that voluntary movements are prepared before they are executed (Kutas and

\footnotetext{
Received April 30, 2015; revised July 3, 2015; accepted July 10, 2015.

Author contributions: J.A.M., B.D., R.W.I., and H.S. designed research; J.A.M., B.D., R.W.I., and H.S. performed research; J.A.M., B.D., and H.S. analyzed data; J.A.M. and H.S. wrote the paper.

This work was supported by German Research Foundation Research Grant SCHE 1575/1-1. We thank Natalie Nazarenus, Ricarda Ahlert, and Matthias Dörge for technical assistance.

The authors declare no competing financial interests.

Correspondence should be addressed to Hansjörg Scherberger, Neurobiology Laboratory, German Primate Center, Kellnerweg 4, D-37077 Göttingen, Germany. E-mail: HScherberger@dpz.eu.

DOI:10.1523/JNEUROSCI.1714-15.2015

Copyright $\odot 2015$ the authors $\quad 0270-6474 / 15 / 3511415-18 \$ 15.00 / 0$
}

Donchin, 1974; Wise, 1985; Ghez et al., 1997). A benefit of longer preparation is a reduction in reaction times (RTs), which is the time between a go signal and the initiation of a movement (Rosenbaum, 1980; Riehle and Requin, 1989). Nevertheless, RT varies even for similar amounts of preparation.

The ideal design for studying motor preparation is the delayed reaching task, in which a movement must be planned and withheld for a certain time. Studies have shown that population activity of neurons in the dorsal premotor cortex (PMd) of the primate brain, recorded either sequentially (Riehle and Requin, 1993) or in parallel (Churchland et al., 2006c; Afshar et al., 2011; Churchland, 2015), can explain a large portion of the variability in reach RT and reach velocity (Churchland et al., 2006a,b). Similar results have been obtained using sequential recordings in the parietal reach region (Snyder et al., 2006) and lateral intraparietal area (Janssen and Shadlen, 2005). However, a comparative study of the frontoparietal network has not been undertaken. 
To analyze RT variability, an understanding of preparatory activity is vital. A number of models have been proposed to explain how preparation of movement is undertaken. Initial models related the preparatory activity of single neurons to behavior by suggesting that subpopulations of neurons may hold activity near a movement threshold that is crossed to initiate movement (Riehle and Requin, 1993; Hanes and Schall, 1996; Erlhagen and Schöner, 2002), whereas more recent models use a state space framework of population activity. In the latter framework, the firing of each neuron represents a dimension in a highdimensional space of all neurons. Hence, the firing of all neurons at a particular time represents a single point in the state space, de-emphasizing the importance of tuning properties of individual neurons (Fetz, 1992). The "optimal subspace" hypothesis posits that a preparatory state is achieved during planning and that deviations from this state may delay movement (Churchland et al., 2006c; Churchland and Shenoy, 2007a). The "initial condition hypothesis" augmented this view by further stating that trial-to-trial fluctuations in the neural trajectory are correlated with RT (Afshar et al., 2011).

To elucidate the role of the frontoparietal network in preparation, the established hand grasping circuit (Luppino et al., 1999) consisting of the hand area (F5) of the ventral premotor cortex (PMv) and the anterior intraparietal area (AIP) were investigated using a delayed grasping task. Neural activity in these areas is modulated strongly by visual object properties (Murata et al., 1997, 2000), extrinsic goals (Kakei et al., 2001), performed grip types (Baumann et al., 2009; Fluet et al., 2010), and preparatory activity in these areas can be used to decode the visual properties of objects and complex hand shapes required to grasp a diverse range of objects (Carpaneto et al., 2011; Townsend et al., 2011; Schaffelhofer et al., 2015).

In the current study, we analyzed population activity in a delayed grasping task with multiple grip types to evaluate how population activity of simultaneously recorded units in F5 and AIP might inform subsequent behavior. Preparatory activity in F5 could explain up to $18 \%$ of the variability in trial-to-trial RT, a significant finding, whereas AIP could explain only up to $6 \%$. By demonstrating a significant advantage of F5 over AIP in RT prediction, our results support the concept that the encoding of RT is represented primarily in the frontal and not the parietal lobe, at least when grasping in the dark.

\section{Materials and Methods}

Basic procedures. Neural activity was recorded simultaneously from area F5 and area AIP in one male and two female rhesus macaque monkeys (Macaca mulatta, animals B, S, and Z; body weight, $11.2,9.7$, and $7.0 \mathrm{~kg}$, respectively). Animal care and all experimental procedures were conducted in accordance with German and European law and were in agreement with the Guidelines for the Care and Use of Mammals in Neuroscience and Behavioral Research (National Research Council, 2003).

Basic experimental methods have been described previously (Townsend et al., 2011; Schaffelhofer et al., 2015). We trained animals to perform a delayed grasping task. They were seated in a primate chair and trained to grasp a handle with the left hand (animals B and Z) or the right hand (animal S; Fig. 1D). This handle was placed in front of the monkey at chest level and in the vertical position at a distance of $\sim 26 \mathrm{~cm}$, i.e., the monkeys had to reach a distance of $26 \mathrm{~cm}$ to grasp the handle. The handle could be grasped either with a power grip (opposition of fingers and palm) or precision grip (opposition of index finger and thumb; Fig. 1E). Two clearly visible recessions on either side of the handle contained touch sensors that detected thumb and forefinger contact during precision grips, whereas power grips were detected using an infrared light barrier inside the handle aperture. The monkey was instructed which grip type to make by means of two colored LED-like light dots projected from a thin-film transistor (TFT) screen (CTF846-A; screen size, 8 inches, digital; resolution, $800 \times 600$; refresh rate, $75 \mathrm{~Hz}$ ) onto the center of the handle via a half mirror positioned between the animal's eyes and the target. A mask preventing a direct view of the image was placed in front of the TFT screen and two spotlights placed on either side could illuminate the handle. Apart from these light sources, the experimental room was completely dark. In addition, one or two capacitive touch sensors (model EC3016NPAPL; Carlo Gavazzi) were placed at the level of the animals' midtorso and functioned as hand-rest buttons. The nonacting arm of animals $\mathrm{B}$ and $\mathrm{Z}$ were placed in a long tube, preventing it from interacting with the handle. Monkey $S$ was trained to keep its nonacting hand on an additional hand-rest button.

Eye movements were measured using an infrared optical eye tracker (model AA-ETL-200; ISCAN) via a heat mirror directly in front of the monkey's head. To adjust the gain and offset, red calibration dots were shown at different locations at the beginning of each session for 25 trials that the animal fixated for at least $2 \mathrm{~s}$.

Eye tracking and the behavioral task were controlled by customwritten software implemented in LabView Realtime (National Instruments) with a time resolution of $1 \mathrm{~ms}$. An infrared camera was used to monitor behavior continuously throughout the entire experiment.

Behavioral paradigm. Animals B and S performed Task 1 (Fig. 1E), whereas animal $\mathrm{Z}$ performed Task 2 (Fig. $1 F$ ). The following is an explanation of the trial course of Task 1 . Trials started after the monkey placed the acting hand on the resting position and fixated a red dot (fixation period). The animal was required to keep the acting hand, or both hands (animal S), completely still on the resting position until after the go cue. After 400-700 ms, two flashlights illuminated the handle for $300 \mathrm{~ms}$, followed by $600 \mathrm{~ms}$ of additional fixation. In the cue period, a second light dot was then shown next to the red one to instruct the monkey about the grip type for this trial (grip cue). Either a green or white dot appeared for $300 \mathrm{~ms}$, indicating a power or a precision grip, respectively. After that, the monkey had to memorize the instruction for a variable memory period. This memory period lasted for $0-1300 \mathrm{~ms}$ (i.e., the go cue could appear simultaneously with the grip cue), in discrete memory period bins of $0,100,200,300,400,500,600,700,800,900,1000$, or $1300 \mathrm{~ms}$, which were pseudorandomly sampled with an equal number of trials from each condition. Regardless of memory period length, the grip cue was always shown for $300 \mathrm{~ms}$. Switching off the fixation light then cued the monkey to reach and grasp the target (movement period) to receive a liquid reward. Animals were required to hold the appropriate grip for $300 \mathrm{~ms}$. Additionally, catch trials were interleaved randomly ( $\sim 8 \%$ of trials), in which a go cue was never shown and the animal only received a reward if it maintained fixation and the hands on the hand rests for $2000 \mathrm{~ms}$ after the grip cue. All trials were interleaved randomly and in total darkness.

The differences between Task 1 and Task 2 are as follows. In Task 2, there was only one fixation period that lasted for 600-1000 ms. In Task 2, the illumination of the handle took place at the time of grip cue. In the instructed version of Task 2 , the grip cues were identical to Task 1 . In the free-choice version, both a green and white dot appeared simultaneously, indicating that the monkey was free to choose between the two grip types. This was followed by a memory period lasting $400-600 \mathrm{~ms}$, and then either the green or white dot reappeared for $300 \mathrm{~ms}$ in $50 \%$ of all free-choice trials, which turned the free-choice task into a delayed-instructed task and was followed by a second memory period (duration, $400-600 \mathrm{~ms}$ ). In all other trials (instructed or free choice), only the red fixation dot was shown during the second cue period, making it impossible to distinguish the first and second memory periods. The hold period in Task 2 was $200 \mathrm{~ms}$ as opposed to $300 \mathrm{~ms}$ in Task 1. Importantly, during free-choice trials, the reward was reduced every time the monkey repeatedly chose the same grip type.

Surgical procedures and imaging. After completion of behavioral training, each animal received an MRI scan to locate anatomical landmarks for subsequent chronic implantation of microelectrode arrays. Each monkey was sedated (e.g., $10 \mathrm{mg} / \mathrm{kg}$ ketamine and $0.5 \mathrm{mg} / \mathrm{kg}$ xylazine, i.m.) and placed in the scanner (GE Healthcare 1.5T or Siemens Trio 3T) in a prone position. T1-weighted volumetric images of the brain and skull were obtained as described previously (Baumann et al., 2009). We measured the stereotaxic location of the arcuate and intraparietal sulci to guide placement of the electrode arrays. 
A

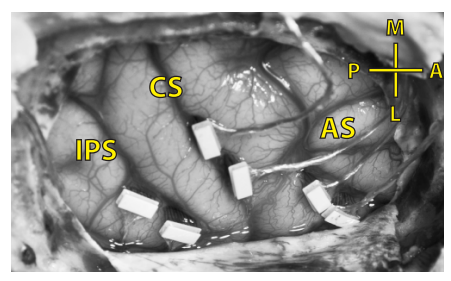

B

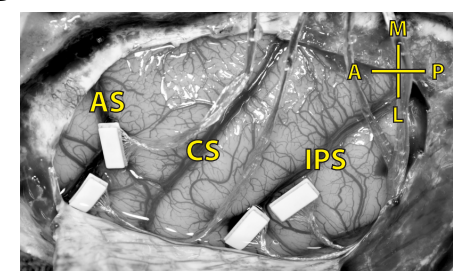

E

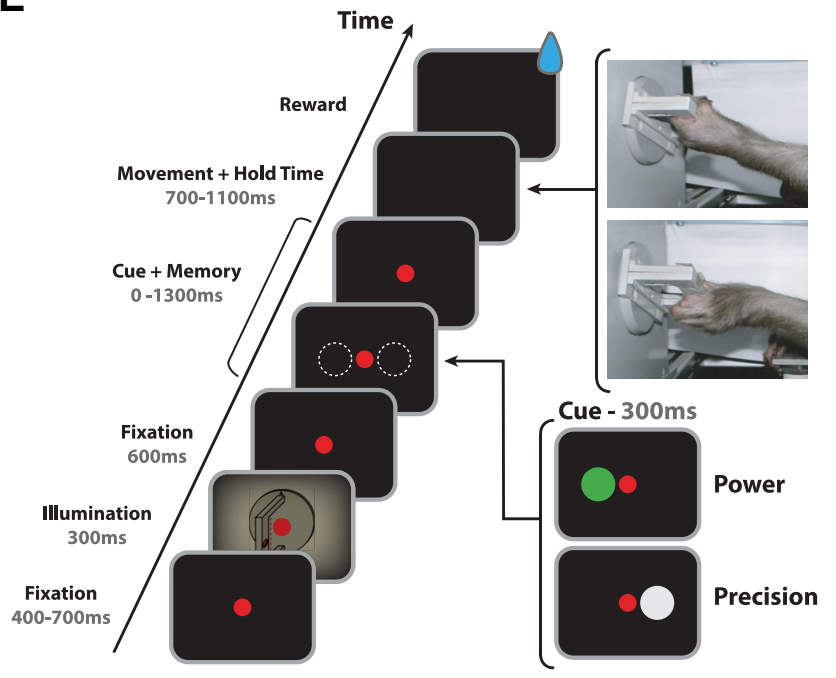

C

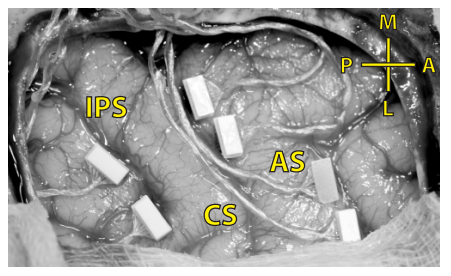

D

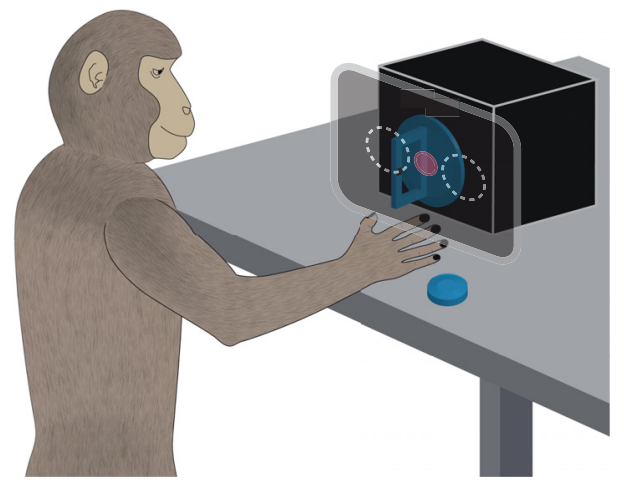

$\mathbf{F}$

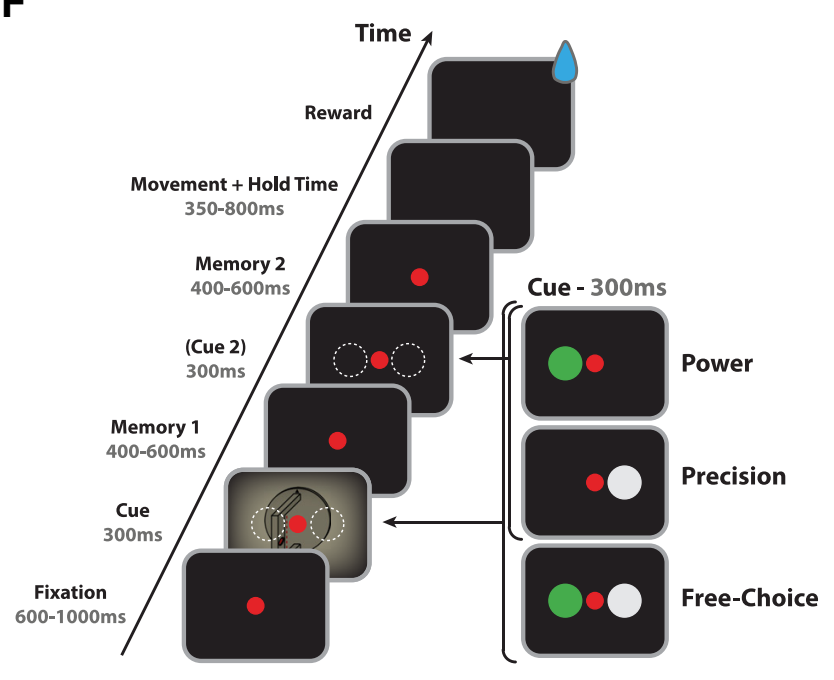

Figure 1. FMA implantation and task design. A-C, Array locations for animals B, S, and Z, respectively. Two arrays were placed in $F 5$ on the bank of the arcuate sulcus (AS). Two additional arrays were placed in AIP toward the lateral end of the intraparietal sulcus (IPS). In animals B and Z, two more arrays were placed on the bank of the central sulcus (CS). The cross shows medial (M), lateral $(\mathrm{L})$, anterior (A), and posterior (P) directions. Note that animal $S$ was implanted in the left hemisphere and animals B and Z in the right hemisphere. D, Sketch of an animal in the experimental setup. The cues were presented on a monitor projected onto a mirror, making the light dots appear superimposed onto the grasping handle. $\boldsymbol{E}$, Delayed grasping task with two grip types (Task 1 ). An example of each grip type can be seen during the movement epoch (top, power grip; bottom, precision grip). The handle was rotated to a supine orientation for demonstration purposes only. $\boldsymbol{F}$, Delayed grasping task with two grip types and three decision conditions (Task 2). Free-choice trials were presented twice as often as each of the other conditions. Delayed-instructed trials contained a second grip cue turning a free-choice trial into a delayed-instructed trial. Trials were presented in a pseudorandom order.

Chronic electrode implantation. An initial surgery was performed to implant a head post (titanium cylinder; diameter, $18 \mathrm{~mm}$ ). After recovery from this procedure and subsequent training of the task in the head-fixed condition, each animal was implanted with floating microelectrode arrays (FMAs; MicroProbes for Life Science) in a separate procedure. Animal $\mathrm{S}$ was implanted with 32 electrode FMAs and received two arrays in each area (Fig. 1B). The arcuate sulcus of animal $S$ did not present a spur, but in the MRI a small indentation was visible in the posterior bank, $\sim 2$ $\mathrm{mm}$ medial to the knee, which we treated as the spur. We placed both anterior FMAs lateral to that mark. Animals B and Z were implanted with six electrode arrays in the right hemisphere, each with 32 electrodes (Fig. $1 A, C)$. Two such arrays were implanted in area F5, two in area AIP, and two in the primary motor cortex (M1). FMAs consisted of nonmoveable monopolar platinum-iridium electrodes with initial impedances ranging from 300 to $600 \mathrm{k} \Omega$ at $1 \mathrm{kHz}$ measured before implantation. Postimplantation measurements in the first months after implantation confirmed these values in vivo. Lengths of electrodes were $1.5-7.1 \mathrm{~mm}$.

All surgical procedures were performed under sterile conditions and general anesthesia (e.g., induction with $10 \mathrm{mg} / \mathrm{kg}$ ketamine, i.m., and $0.05 \mathrm{mg} / \mathrm{kg}$ atropine, s.c., followed by intubation, $1-2 \%$ isoflurane, and analgesia with $0.01 \mathrm{mg} / \mathrm{kg}$ buprenorphene, s.c.). Heart and respiration rates, electrocardiogram, oxygen saturation, and body temperature were monitored continuously, and systemic antibiotics and analgesics were administered for several days after each surgery. To prevent brain swelling while the dura was open, the animal was hyperventilated mildly (end-tidal $\mathrm{CO}_{2}, \sim 30 \mathrm{mmHg}$ ), and mannitol was kept at hand. Animals were allowed to recover fully ( $\sim 2$ weeks) before behavioral training or recording experiments commenced.

Neural recordings and spike sorting. Signals from the implanted arrays were amplified and stored digitally using a 128 channel recording system (sampling rate, $30 \mathrm{kS} / \mathrm{s} ; 0.6-7500 \mathrm{~Hz}$ hardware filter; Cerebus; Blackrock Microsystems). Data were first filtered using a median filter (window length, $3 \mathrm{~ms}$ ), and the result was subtracted from the raw signal. Afterward, the signal was low-pass filtered with a causal Butterworth filter (5000 Hz; fourth order). To eliminate movement noise (i.e., common component induced by reference and ground), principal component analysis (PCA) artifact cancellation was applied for all electrodes of each array (as described by Musial et al., 2002). To ensure that no individual channels were eliminated, PCA dimensions with any coefficient $>0.36$ (with respect to normalized data) were retained. Spike waveforms were 
extracted and semiautomatically sorted using a modified version of the offline spike sorter Wave_clus (Quiroga et al., 2004; Kraskov et al., 2009).

Unit isolation was evaluated using four criteria: (1) the absence of short (1-2 ms) intervals in the interspike interval histogram for single units, (2) the degree of homogeneity of the detected spike waveforms, (3) the separation of waveform clusters in the projection of the first $17 \mathrm{fea}-$ tures detected by Wave_clus, and (4) the uniqueness of the shape of the interspike interval distribution.

After the semiautomatic sorting process, redetection of the average waveforms (templates) was done to detect overlaid waveforms (Gozani and Miller, 1994). Filtered signals were convolved with the templates starting with the biggest waveform. Independently for each template, redetection and resorting was run automatically using a linear classifier function (MATLAB function classify). After the identification of the target template, the shift-corrected template (achieved by up and down sampling) was subtracted from the filtered signal of the corresponding channel to reduce artifacts for detection of the next template. This procedure allowed a detection of templates up to an overlap of $0.2 \mathrm{~ms}$. As a control, unit isolation was evaluated again as described previously to determine the final classification of all units into single units or multiunits. In case of ambiguity, a unit was not classified as single. Stationarity of firing rate was checked for all units, and, in case the firing rate was not stable over the entire recording period ( $>30 \%$ change in firing rate between the first $10 \mathrm{~min}$ and the last $10 \mathrm{~min}$ of recording), the unit was excluded from additional analyses ( $<3 \%$ of all single units).

Data preprocessing. In all datasets trials with outlying RTs, $>700 \mathrm{~ms}$ in Task 1 and $>500 \mathrm{~ms}$ in Task 2 and $<200 \mathrm{~ms}$ in either task were excluded. In animals $\mathrm{B}$ and $\mathrm{S}$, these trials comprised $<1 \%$ of the data and $<3 \%$ in animal Z. Clearly, all animals were careful to wait for the appropriate go cue and did not act preemptively. We used this conservative check on outlier RTs to safely exclude the possibility that animals were acting in anticipation of the go cue.

Crucially, for all analyses of Task 1, trials with memory periods $<500 \mathrm{~ms}$ were excluded from analysis. These short memory period trials were removed to ensure that animals had sufficient time to fully plan the movement before acting. Such an exclusion criteria was not used in Task 2, because the animal never had $<700 \mathrm{~ms}$ to plan (delayed-instructed condition) and was trained for many months to acquire this timing scheme.

All recorded units (single unit and multiunit) were used in our main analyses. After spike sorting, spike events were binned in overlapping 100 ms windows and sampled every millisecond to produce a continuous firing rate signal $(1 \mathrm{kHz})$. This means that firing rates at the time of the go cue considered spikes occurring $50 \mathrm{~ms}$ before to $50 \mathrm{~ms}$ after the go cue. Because it is unlikely that (sensory) responses to the go cue would be represented in AIP or F5 already at 50 ms after presentation, we believe this binning does not bias the predictive power of RTs. In fact, our conclusions do not change when using a binning that does not extend beyond the go cue (data not shown).

Dimensionality reduction. Dimensionality reduction was performed for the purposes of visualization only. All quantitative analyses relied on the full dimensionality of the data. Gaussian-process factor analysis (GPFA) was performed on the neural data from cue presentation to movement onset (Yu et al., 2009). This method performs smoothing of spike trains and dimensionality reduction simultaneously within a common probabilistic framework. It assumes that the activity of each unit is a linear function (plus noise) of a low-dimensional neural state whose evolution in time is well described by a Gaussian process. This methods allows for better visualization on the single-trial level than other published methods (Yu et al., 2009). The data were reduced to 12 dimensions (the optimal number of latent dimensions in the data as determined by cross-validation) using $20 \mathrm{~ms}$ nonoverlapping spike bins to produce the trajectories in Figure $3 A$. In this reduction, the three displayed dimensions explain $63 \%$ of the total variance. In this figure, a rotation of the first three latent dimensions is shown (equivalent to a linear combination of the three dimensions explaining the most variance overall).

Similarly, neural trajectories in Figure 9 were generated by performing PCA on the peristimulus time histograms of all units for each grasp condition separately. All individual trials were then transformed into the two principal components explaining the most variance and binned into slow, medium, and fast RTs. All trials were aligned to the go cue and plotted from $350 \mathrm{~ms}$ before to $280 \mathrm{~ms}$ after the go cue.

Projection methods. As can be seen in Figure $3 A$, trials of the same condition tend to follow a stereotypical trajectory through neural space. Following the study by Afshar et al. (2011), we reasoned that the farther the neural state had advanced along the mean neural path at the time of the go cue would be predictive of subsequent performance. To test this hypothesis, we projected neural activity of individual trials at the go cue on the mean neural trajectory of similar trials (excluding the tested trial of the same condition). The projection is denoted in Figure $3 B$ with the symbol $\alpha$. The vector formed between the mean firing rate at the go cue and the firing rate at the go cue of an individual trial is projected onto the vector between the mean firing at go and the mean firing at go \pm some $\Delta t$. The data were tested empirically to determine the optimal $\Delta t$ values over all datasets. Selected $\Delta t$ values ranged from $300 \mathrm{~ms}$ before to $300 \mathrm{~ms}$ after the go cue.

Additionally, as depicted in Figure 3D, the instantaneous velocity of the neural data, $\left[t_{\mathrm{go}}-\left(t_{\mathrm{go}}-20\right)\right]$, in the high-dimensional neural space of individual trials was projected onto the mean neural trajectory. Similar to the projection method, the velocity projection method hypothesized that trials in which the neural space is changing in the direction of the mean trajectory will have shorter RTs. Importantly, trials were segregated into $100-200 \mathrm{~ms}$ bins based on the length of the memory period to minimize the effect of memory period length on neural position, i.e., the mean trajectory used as a reference for each trial was calculated solely on other trials within the same memory period bin.

Euclidian distance method. The Euclidian distance method was performed also equivalently to the study by Afshar et al. (2011). Single-trial RT was correlated with the Euclidian distance between the highdimensional firing rate at the go cue on the single trial and the mean high-dimensional firing rate of all other trials of the same condition at some time offset, $\Delta t$, as depicted in Figure $3 C$.

The optimal subspace method, as originally reported by Churchland et al. (2006c), was also performed. It is equivalent to the Euclidian distance method with a time offset of $\Delta t=0 \mathrm{~ms}$. Both of these methods are based on the hypothesis that trials in which firing rates are close to the mean rates observed for similar trials have shorter RTs.

Average rate method. The average rate (AR) method is based on the simple hypothesis that trials during which particular units have higher firing rates will be associated with shorter RTs. This method posits that neural activity increases during preparation and crosses a movement threshold to initiate a movement, also known as the rise-to-threshold hypothesis (Erlhagen and Schöner, 2002). Under the assumptions of this method, higher preparatory activity would always be associated with shorter RTs. Four implementations of this method were tested initially. The trial-by-trial RT was correlated with the following: (1) the signed difference between firing rate at go cue and at cue onset (i.e., an approximation of baseline firing), averaged across all units; (2) the same method but using the unsigned difference (absolute value); (3) the average firing rate at the go cue across all units; and (4) the average firing rate at the go cue across all units for their preferred grip type only. The third version, which does not rely on baseline firing rate or unit preferences, was the best performing (data not shown) and was therefore the one used for additional analysis. For clarity, we opted to name our implementation of the rise-to-threshold hypothesis as the AR method.

Sign-corrected average rate method. As hypothesized by the AR method, if units that increase their activity (relative to the mean) during movement preparation are associated with trials having short RTs, then they are negatively correlated with RT. However, if the activity of some units were in fact reduced (relative to the mean) for trials with short RT, this would result in a positive correlation. If many of each of these types exists in the same population, which is averaged to produce an RT prediction, these two inverted populations would be in conflict and cancel out each other, thereby causing poor RT prediction.

To overcome this obstacle, we introduced the sign-corrected average rate (SCAR) method. It is identical to the simple AR method as described in the previous section; however, the signal of all units was first multiplied with a sign-correction vector. That is, units that were correlated positively with RT were inverted to produce a negative correlation. To 
A

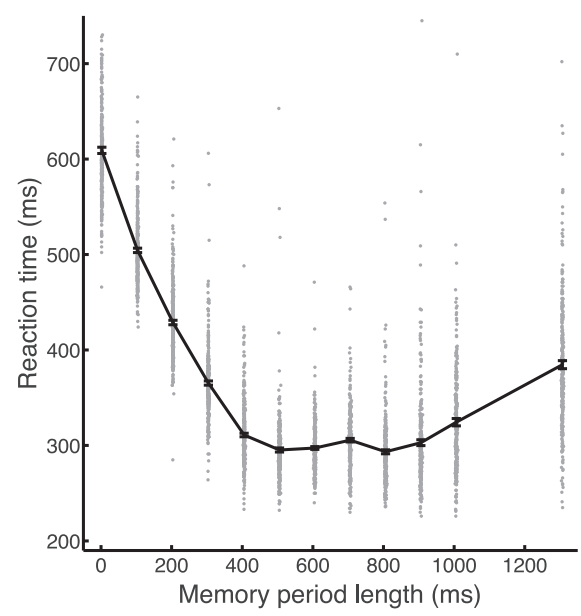

B

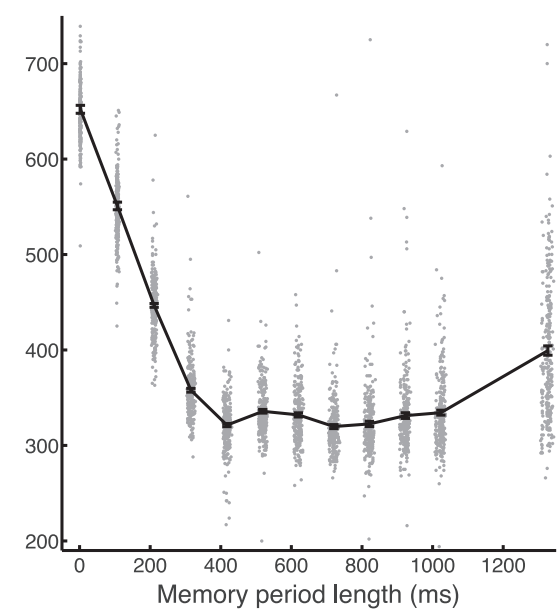

C

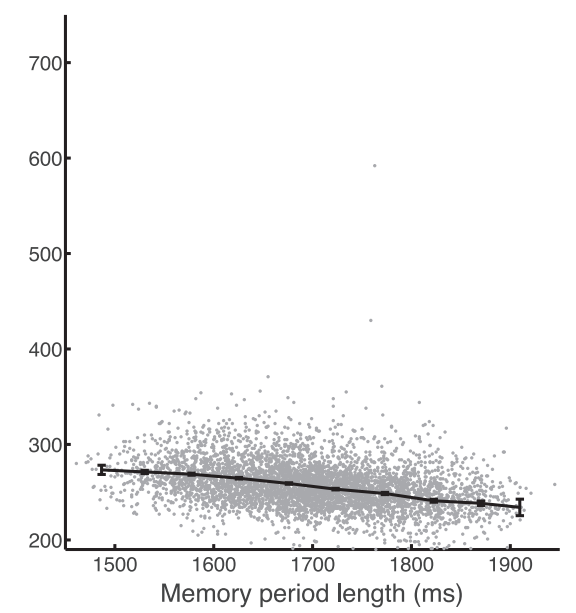

Figure 2. Scatter plots of RT versus memory period length. $A-C$, The RT of animals B, S, and Z, respectively, as a function of memory period length for all task conditions and datasets. The solid line represents the mean, and error bars indicate SEM within nonoverlapping $50 \mathrm{~ms}$ bins.

decide which units were to receive a negative value in the sign-correction vector, (1) the firing rates at the go cue of individual units were correlated with RT (twofold cross-validated) over all conditions. (2) Units received a -1 value in the sign-correction vector if they possessed on average (over all conditions) an $r$ value $>0$. All other units received a value of 1 in the correction vector. This method preserves the absolute magnitude of the mean firing rate across trials because no normalization is performed. A number of inversion criteria were tested; however, we found that it was sufficient to invert only units with a positive $r$ value (data not shown). It is important to note that units were not tested separately for each condition (grip type/decision condition), i.e., a unit that was inverted for a precision grip would also be inverted during a power grip. Testing on each condition separately would have increased RT prediction further.

$R T$ correlations. When correlating single-trial neural metrics, i.e., the previously described methods excluding the AR and SCAR methods, with RT, we did not include the neural data from that trial in the calculation of the mean neural trajectory used for that prediction, as in the study by Afshar et al. (2011). The predicted and observed RTs were then correlated with each other. This technique, termed leave-one-out crossvalidation, ensured that predictions of the RT of each trial were not based on movement activity from that trial.

Whenever average RT variance explained was calculated across an average of datasets, each average was weighted by the number of trials in each dataset.

Partial RT correlations. In our tasks, memory period length was correlated highly with RT (Fig. 2). To disentangle the relationship of memory period length to RT and the relationship of our neural prediction metrics on RT, partial correlation, which bares much similarity to multiple regression, was performed (Cramér, 1946). Partial correlation is a method for determining the correlation between two variables while controlling for one or more other variables. The partial correlation between two variables, while controlling for a single other variable, is described by

$$
\rho_{N B \times M}=\frac{r_{N B}-r_{N M} r_{M B}}{\sqrt{1-r_{N M}^{2}} \sqrt{1-r_{M B}^{2}}},
$$

where $\rho$ is the partial correlation of a neural prediction metric $(N)$ with RT $(B)$, while controlling for the effect of the length of the memory period $(M) . r_{X Y}$ is the standard Pearson's correlation between vectors $X$ and $Y$.

Cross-validation. The results of all methods were twofold crossvalidated. All trials of each dataset were first randomly segregated into two sets of equal size and methods performed separately on each set. Furthermore, the SCAR method required the preevaluation of preparatory correlations with RT to determine which units should have their firing rates inverted. To avoid false-positive results, SCAR was first trained on a training set of trials and always tested on trials that were not used for training. All analyses were twofold cross-validated by flipping the role of both sets. Segregating the data into more than two crossvalidation folds would severely reduce the number of test trials in each condition and therefore the reliability of prediction.

Multiple linear regression. To determine whether a combination of the tested methods could improve the amount of variance explained in RT, a number of regressions was performed. Multiple regression was performed using the leave-one-out technique, in which regressing on all other trials generated the prediction for each trial, and this process was repeated for each individual trial. First, the same model as described by Afshar et al. (2011) was used, which consisted of the projection method on both the pre-go and post-go cue axes, as well as the velocity projection method on both the pre-go and post-go cue axes. Alternatively, a number of simpler combinations were tested, although most are not presented here because they yielded poor results.

To test whether or not a multivariate model could explain significantly more variance than a simpler model, the $F$ test was used. The $F$ test is ideally suited to compare models (regressions) that use nested predictors, that is, models that use a subset of predictors of a more complex model. However, because we wanted to compare models over a number of conditions (each with varying degrees of freedom), we had to generate a nonstandard $F$ distribution for testing. Therefore, the $F$ statistic comparing each pair of models was calculated separately and then summed. Additionally, because each cross-validation fold contained different trials, each fold was considered as a separate condition for a total of four conditions in Task 1 (two behavioral, two folds) and 12 conditions (six behavioral, two folds) in Task 2. To generate a testing distribution the probability density functions (pdfs) of each corresponding $F$ statistic were convolved with each other to form a new distribution. We then calculated the likelihood of observing the calculated sum of $F$ statistics and from there derived the $p$ value.

To extend this test over all datasets and reach general conclusions, the sum of $F$ statistics was summed across all conditions and datasets and tested on an $F$ distribution of convolved pdfs over all conditions and datasets.

Chance-level calculation. Many individual correlations were computed in the current study. To ensure that all relevant methods were truly identifying relationships between neural data and RT, all correlations were tested against a chance distribution. For each method and condition, chance distributions were generated by correlating the prediction of each method with a corresponding vector of randomly shuffled RTs (1000 repetitions). We could then calculate the probability of observing the empirical $R^{2}$ given our shuffled distributions and use this as a $p$ value. 
In the case in which a significance calculation was required over multiple conditions and datasets, the generated chance distributions were convolved with each other to form a new distribution, precisely as with the $F$ statistic for testing multiple linear regression. The $p$ value for significance was fixed at 0.01 .

For the SCAR method, an additional control was performed. The SCAR method involved the inversion of the firing rates of some units. To ensure that this inversion did not artificially produce our results, the following control was performed. A random sample of units of the same size as in the real data was inverted and the method performed as normal (1000 repetitions, permutation test). The resulting chance-level distributions could be tested against the empirical results as was done for the other chance-level calculations.

Variance selection. All recorded units were included in the main analyses. To determine whether one could select a subset of units that would perform equally or better than the entire population, a variance selection was performed. The units with higher variances in spike count (at the go cue) across trials were preferentially included first. In addition, a random unit selection was performed alongside the first analysis with the same number of units per test. The random selection of units was performed 1000 times per percentage value. Data were interpolated to the range of $0-100 \%$ to facilitate averaging between datasets.

Significance testing was performed by summing the $R^{2}$ over all datasets and testing the likelihood of obtaining this value against the distribution of convolved pdfs over all datasets as generated by the random unit selection, precisely as was done with the $F$ statistic for testing multiple linear regression. The significance level was set at 0.05 and Bonferroni's corrected for the number of percentages tested (100).

Bayesian information criterion. The Bayesian information criterion (BIC) is a well known model selection criterion (McQuarrie and Tsai, 1998). It is described by the following:

$$
\mathrm{BIC}=-2 \ln \hat{L}+p \ln N,
$$

where $\hat{L}$ is the posterior likelihood of the data given the best-fit model, $p$ is the number of parameters used to generate the model, and $N$ is the number of observations used. A smaller BIC is associated with a better explanatory model. BICs were calculated for single conditions and averaged either over conditions or over conditions and datasets.

\section{Results}

\section{Behavior}

All three animals performed the task successfully. After initiating trials to the point of obtaining task information, i.e., receiving a grip cue, animals $\mathrm{B}, \mathrm{S}$, and $\mathrm{Z}$ successfully completed those trials 96,98 , and $95 \%$ of the time, respectively. Catch trials in which the animal was required to withhold movement were included in Task 1. Animals B and S completed these catch trials successfully 95 and $98 \%$ of the time, respectively. Figure 2 plots the RTs of all animals as a function of memory period length. The memory period in Task 1 lasted $0-1300 \mathrm{~ms}$, whereas the memory period in Task 2 was relatively longer (a minimum of $1400 \mathrm{~ms}$ in the instructed condition including the grip cue) to facilitate a second cue period in the delayed-instructed condition. RTs were reduced during longer memory periods, consistent with the established hypothesis that motor preparation improves over time (Rosenbaum, 1980; Riehle and Requin, 1989). The exception to this was the 1300 ms memory condition in Task 1, in which RT slightly increased, likely because of the expectation of a catch trial, which appeared periodically and lasted $2000 \mathrm{~ms}$. For animals B, S, and Z, the correlation coefficients over all datasets between memory period length and RT were $-0.55,-0.57$, and -0.33 , respectively. Similar experiments have shown that saturation of RT, i.e., the minimum length of memory period after which RT does not significantly improve, is at least 100-200 ms (Churchland et al., 2006c) in a reaching task. In Task 1, we observed RT saturation, but we did not observe this in Task 2 .
In contrast, there was no significant correlation between memory period length and movement time, which is the time between the hand leaving the hand-rest button and making contact with the handle, indicating that animals only initiated a movement when the movement was fully prepared. The only exceptions are the movement times of animal $\mathrm{B}$, which were slightly longer in the $1300 \mathrm{~ms}$ memory period condition than in shorter memory periods, potentially an effect of decreased attention for long memory periods. For all animals, the hands remained completely stationary on the hand-rest buttons before the go cue. The experiments from which these data were collected were originally designed to assess hypotheses that are not presented here and will be addressed elsewhere.

\section{Neural recordings}

The analyzed datasets include a collection of 18 recording sessions, six from each animal. In animal $B$, an average \pm SD of $63 \pm$ 17 and $28 \pm 18$ units were recorded in F5 and AIP, respectively, as well as in animal S (mean \pm SD, $132 \pm 15$ and $131 \pm 26$ ) and animal $Z(85 \pm 18$ and $81 \pm 24)$. An average of 483 trials per dataset met the inclusion criteria, as described in Materials and Methods. This corresponded to an average of 77 trials per condition and cross-validation fold overall. In animals $S$ and $\mathrm{Z}$, there was no significant difference between the two brain regions in the number of units recorded per dataset $(p=1$ and $p=0.56$, Wilcoxon's signed-rank test). However, in animal B, significantly more units were obtained in area F5 $(p=0.03)$, which may have affected the quality of RT decoding in area AIP. The majority of units obtained in all animals were identified as multiunits (52\% in animal B, 60\% in animal S, 70\% in animal Z). All recorded single units and multiunits were included in additional analyses.

Although the response characteristics of each individual unit are not analyzed here in detail, it is worth noting that the overall tuning characteristics of units in F5 and AIP were very similar regardless of the task design used (Task 1 or 2). Furthermore, both tasks were able to elicit strong grip type tuning in both F5 and AIP. An average of $32 \%$ of recorded units were significantly tuned for grip type during the late memory period in F5, whereas $26 \%$ were tuned in AIP $(p<0.05$, two-sample $t$ test $)$, which did not differ between areas ( $p=0.09$, Kruskal-Wallis ANOVA), although differences were seen between animals $(p=0.002$, Kruskal-Wallis ANOVA), with animal B showing slightly less tuning overall (24\% in F5 and $18 \%$ in AIP). This finding is particularly robust when considering that there are no visual cues present in the memory period, and, therefore, grip type tuning tends to reach a minimum during this epoch.

\section{Low-dimensional visualization of single-trial trajectories}

To visualize how neural data evolves on single trials, a lowdimensional representation of the full neural space of both brain areas combined is shown in Figure $3 A$ for an exemplar dataset (instructed precision grip, dataset Z120829). Dimensionality reduction was performed using GPFA, as described in Materials and Methods. Single trials tended to evolve from cue onset to a preparation state and further to a movement state after the go cue. Conversely, it did not appear that variability between trials decreased in a systematic way when comparing the size of the confidence ellipses at cue onset, go cue, and movement onset. To determine whether the trajectory of an individual trial could be related to RT, three methods were formulated, as depicted in Figure $3 B-D$. These three methods, the projection method, Euclidean distance method, and velocity projection method, are presented here virtually identical to how they were performed by 
A

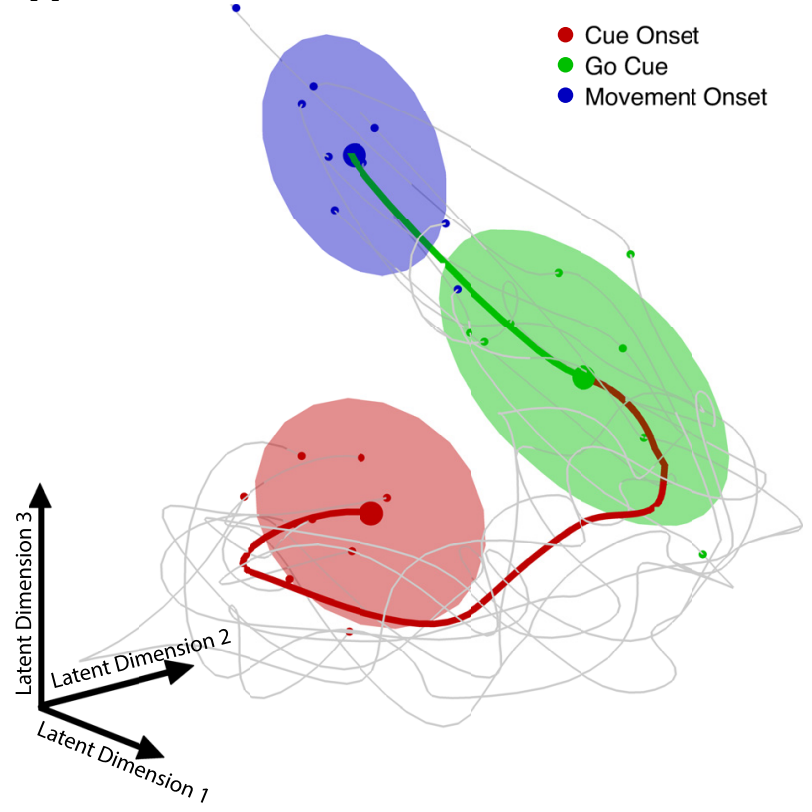

B

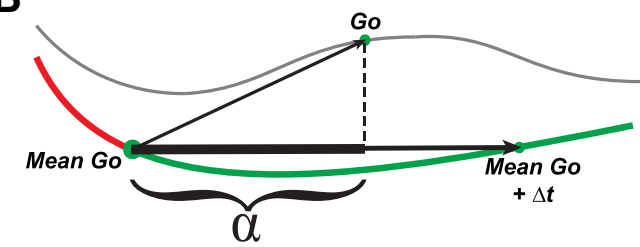

C

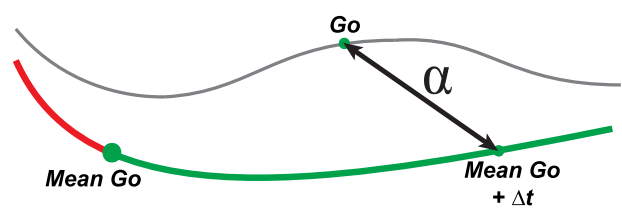

D

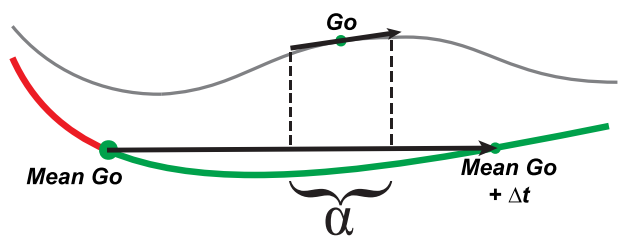

Figure 3. Neural data and RT prediction methods visualized as low-dimensional trajectories. $A$, Neural data of both areas of an exemplar condition reduced to a low-dimensional representation of the trial course (determined by GPFA). Thick trace represents the mean of trials for one condition (instructed precision grip, dataset Z120829). Thin gray traces represent 10 random single trials. Shaded ellipses ( $90 \%$ confidence) represent the state of all selected single trials at the start of each epoch. $\boldsymbol{B}-\boldsymbol{D}$, High-dimensional RT prediction methods in a two-dimensional illustration. Thick red and green traces represents the mean of trials. Thin gray trace represents a single exemplar trial. $\alpha$ denotes the component used to predict RT for the projection method ( $\boldsymbol{B}$ ), Euclidean distance method $(\boldsymbol{C})$, and velocity projection method (D).

Afshar et al. (2011) and are described in detail in Materials and Methods. Although GPFA aids the visualization of single trials, in the following section, these RT prediction methods are tested in the high-dimensional state space of all recorded units.

\section{Finding optimal reference points for trajectory-based methods}

As can be seen in Figure $3 B-D$, each of the three highdimensional state space methods relies on a reference time point, or $\Delta t$, on the mean trajectory. To find the optimal reference point, the time domain from $500 \mathrm{~ms}$ before to $500 \mathrm{~ms}$ after the go cue was tested. For purpose of determining optimal $\Delta t$ values, this time window was further limited to $\pm 300 \mathrm{~ms}$, because reference points become more unreliable between conditions and datasets at large offsets. Each method was performed with this range of $\Delta t$ values, and the predictions correlated with $\mathrm{RT}$, as seen in Figure 4 . The mean of all conditions is shown with a thick trace, and the used offsets before and after the go cue are marked with open circles (limited within $\pm 300 \mathrm{~ms}$ ). Inset histograms show all individual correlation coefficients [datasets $(6) \times$ conditions $(2-6) \times$ cross-validation folds (2)] before squaring and averaging and for each animal separately. The darker bars indicate correlations that are statistically significant $(p<$ 0.05 , Pearson's correlation). The results from animals B and $\mathrm{Z}$ are very similar and use identical offsets, whereas the results of animal S differed significantly.

As seen in Figure $4 A$, the projection method using references both before and after the go cue have correlation distributions with nonzero median in F5: one distribution is shifted to the negative and one to the positive. This finding is consistent with our hypothesis, because trials that are farther along a mean trajectory going forward in time should lead to shorter RTs and therefore an overall negative correlation between our neural predictor and RT. In AIP the projection method also performed significantly, although the resulting $R^{2}$ is much lower than in F5 (Fig. $4 B$ ). Based on this analysis, the best positive and negative $\Delta t$ values, which were then used in all subsequent analysis, were -290 and $60 \mathrm{~ms}$ in F5 for animals B and $\mathrm{Z}$ and -170 and $260 \mathrm{~ms}$ for animal S. In AIP, values of -210 and $200 \mathrm{~ms}$ were used for animals $B$ and $Z$, and values of -40 and $60 \mathrm{~ms}$ were used for animal S.

The Euclidian distance method performed similarly to the projection method but explained overall less variance in RT (Fig. $4 C, D)$. In both F5 and AIP, reference points generally produced correlation histograms that were shifted significantly from zero. In most cases the pre-go distribution was shifted to the negative direction and the post-go to the positive direction, again consistent with the hypothesis that trials that are closer to the state of the network after the go cue will have shorter RTs, with the notable exception of animal B on the pre-go axis, a point that is returned to later. Additionally, when using a time offset of $0 \mathrm{~ms}$, identical to the so-called optimal subspace method (Churchland et al., 2006c), the correlation distribution tended to be only marginally significantly shifted from zero in F5 and AIP. Despite this, the optimal subspace method was not used in additional analyses, because the Euclidean distance method outperformed it in every case. Based on this analysis, the $\Delta t$ values that were used in additional analysis were -300 and $170 \mathrm{~ms}$ in F5 for animals B and Z and -270 and $270 \mathrm{~ms}$ for animal S. In AIP, values of -90 and 300 $\mathrm{ms}$ were used for animals $B$ and $Z$ and values of -100 and $300 \mathrm{~ms}$ for animal S.

The velocity projection method performed poorly overall, accounting for $<1 \%$ of the variance in RT (Fig. $4 E, F$ ). Only rarely do reference points in F5 or AIP have correlation distributions significantly shifted from zero. Furthermore, accounting for the effect of memory period length on RT using partial correlation completely eliminates this effect (data not shown). Therefore, for most of our additional analyses, the velocity projection method 
A

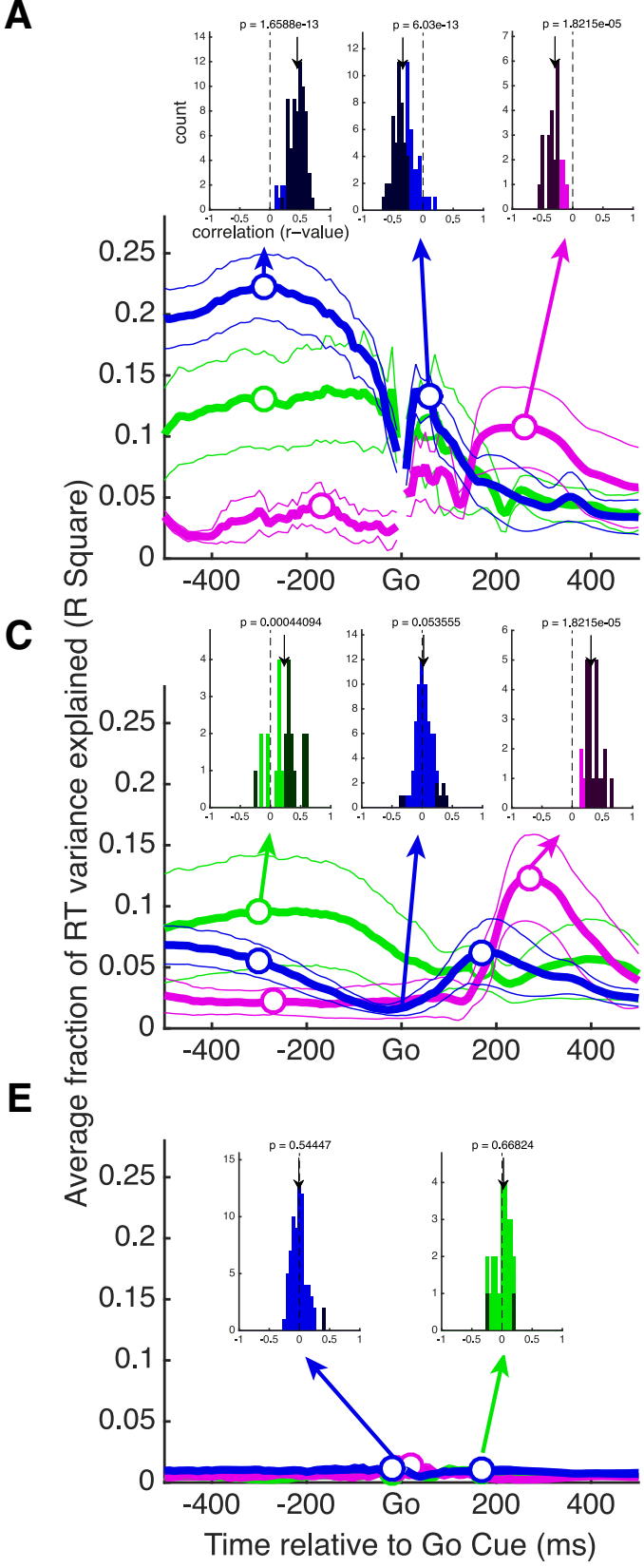

G
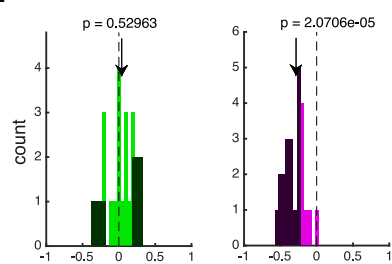

H
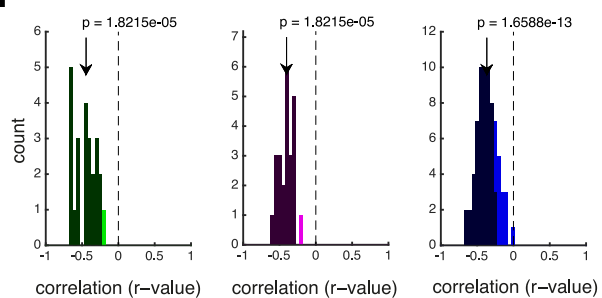

B
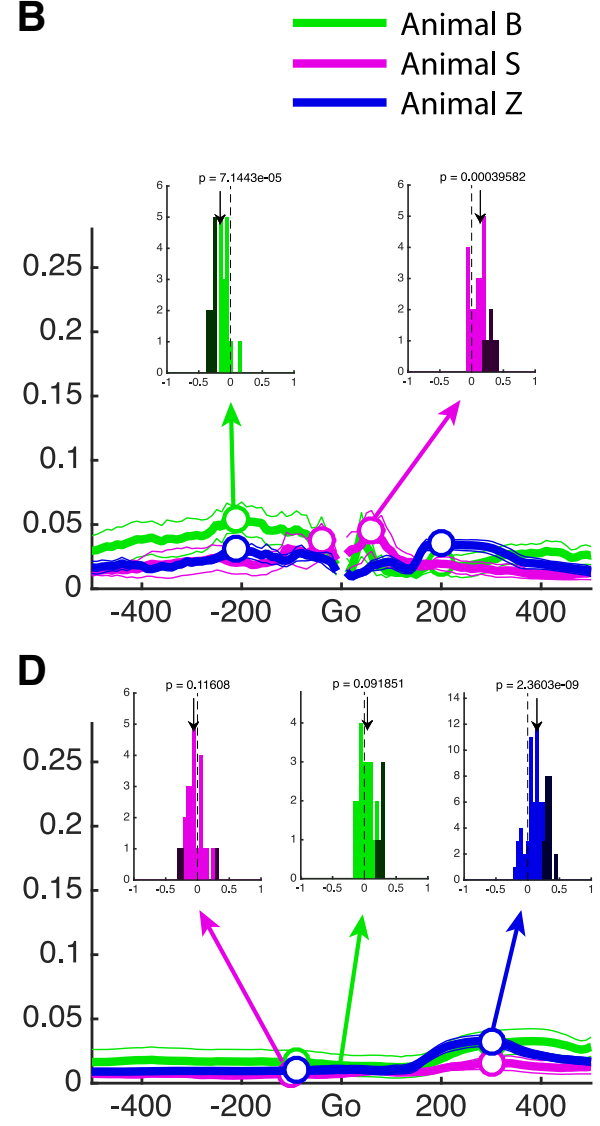

F
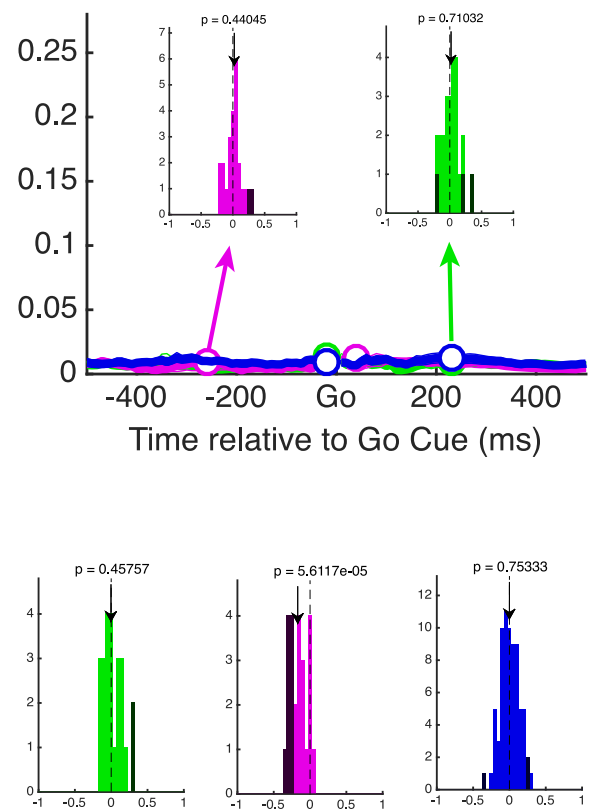

J
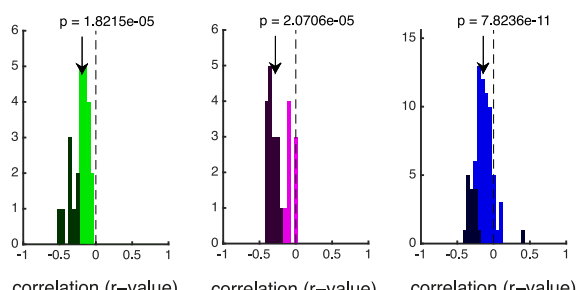
was excluded. It should be noted that trials were segregated into bins based on memory period length, as described in Materials and Methods. However, when all trials are pooled together, the resulting predictions of RT are still nonsignificant (data not shown).

\section{Population firing rate-based methods}

In addition to our high-dimensional trajectory-based methods, we also tested simpler methods based on averaging the activity of all units around the go cue. Such methods still depend on simultaneously recorded units, because they require an estimation of the population neural state for each trial. The first method we tested is the AR at go, which is our implementation of the riseto-threshold hypothesis, as described by Afshar et al. (2011). The correlation histograms obtained by the AR method are shown in Figure 4, $G$ for F5 and $I$ for AIP. The median of the correlation distribution is significantly shifted negatively in two of the three animals in F5 (Wilcoxon's signed-rank test), suggesting that higher firing rates around the go cue led to shorter RTs. However, in AIP, the distribution was only shifted for one of the three animals. However, in all cases in which a significant shift was present, this shift was in the negative direction, suggesting that higher firing rate tended to be related to shorter RTs.

The AR method relies on averaging. Therefore, if some units in the population are correlated negatively with RT whereas others are correlated positively, these effects could cancel out at the population level. To deal with this issue, we first correlated the firing rate at the go cue of each unit with RT on a set of training trials. Then, as described in Materials and Methods, on a set of testing trials we inverted the firing rates of units that had a positive correlation in the pretesting (twofold cross-validated). Briefly, the process consists of multiplying the firing of all units by a sign-correction vector (see Materials and Methods). This new method was termed the SCAR method. The correlation histograms of the SCAR method are shown in Figure 4, $H$ and $J$, for F5 and AIP, respectively. In both areas the median of the correlation distribution was shifted strongly into the negative domain (three of three animals in both areas, Wilcoxon's signed-rank test). Over all datasets, the average number of units whose activity was inverted was $38 \%$ in F5 and $42 \%$ in AIP, a large portion of the total unit count. The number of units inverted was less for animal S, in which the performance of the AR method was already considerably high.

\section{Pooling of multiunits and single units does not bias RT prediction}

To ensure that the previous results were not attributable to the sole contribution of either multiunits or single units, we repeated the analysis using only multiunits or single units. Results are presented as a performance ratio of average fraction of RT vari-

\footnotetext{
$\leftarrow$

Figure 4. Determination of the optimal reference time $(\Delta t)$ relative to go cue on the mean trajectory. $\boldsymbol{A}, \boldsymbol{B}$, Results of the projection method in areas $F 5$ and AIP, respectively. $\boldsymbol{C}, \boldsymbol{D}$, Results of the Euclidean distance method in areas $F 5$ and AIP, respectively. $\boldsymbol{E}, \boldsymbol{F}$, Results of the velocity projection method in areas $F 5$ and AIP, respectively. Thick traces are the mean of all conditions and datasets of each animal, thin traces are the SEM, and white circles are the optimal $\Delta t$ used in all subsequent analysis. Insets in $\boldsymbol{A} \boldsymbol{F}$ show histograms of correlation coefficients between each neural predictor and RT over all conditions (2-6), datasets (6), and cross-validation folds (2). Black bars denote correlations with a $p$ value $<0.05$. Arrows show the median together with the $p$ value of significant difference from zero (Wilcoxon's signed-rank test). $\mathbf{G}, \boldsymbol{H}$, Correlation coefficient histograms of the AR at go method and the SCAR method, respectively, in F5. $\boldsymbol{I}, \boldsymbol{J}$, Same as $\boldsymbol{G}$ and $\boldsymbol{H}$, but for neural data from AIP.
}

ance explained using only single units or multiunits versus the pool of all units (where 1 represents identical performance). In F5, when including only single units, the pre-go and post-go projection methods produced ratios of 0.81 and 0.84 , respectively. In AIP, the pre-go and post-go projection methods produced ratios of 0.76 and 0.91 , respectively. The pre-go and post-go Euclidean distance methods produced ratios of 0.65 and 0.95 for F5 and 0.72 and 0.81 for AIP single units.

When including only multiunits, in F5, the pre-go and post-go projection methods produced ratios of 0.64 and 0.69 in F5 and 0.77 and 0.69 in AIP. The Euclidean distance methods produced ratios of 0.87 and 0.87 in F5 and 1.13 and 0.59 in AIP.

The same analysis was done for the population-based methods (AR and SCAR). The AR method had performance ratios of 0.74 and 0.79 when using single units only in F5 and AIP, respectively, whereas multiunit only performance was 1.25 and 1.07 . The SCAR method had performance ratios of 0.76 and 0.87 when using single units only in F5 and AIP, respectively, whereas multiunit only performance was 0.66 and 0.63 .

In almost every case, including only multiunits or single units in the analysis reduced the overall performance. Using only single units caused a performance reduction of $9-36 \%$. Similarly, using multiunits caused a reduction of $13-41 \%$, with the exception of the Euclidean distance method (before go cue) in AIP and the AR method in both areas, which increased slightly. Overall, the prediction of RT cannot be explained based solely on the contribution of either single units or multiunits. However, the AR method seems to perform best using multiunits, suggesting that compounding single-unit responses stabilizes the performance of this method.

\section{Neural activity predicts trial-by-trial RT}

Together, the results of all methods based on optimal $\Delta t$ selection are shown in Figure 5. The mean $R^{2}$ is plotted for all 18 datasets in both F5 and AIP. Open bars mark methods that did not perform above chance level, as described in Materials and Methods. The average $R^{2}$ over all methods is significantly higher in F5 than AIP $(p<0.001$, Kruskal-Wallis ANOVA), suggesting that the population activity in F5 better encodes the variability in grasping plans. However, it is important to note that the current tasks include a large reaching component, which is also represented strongly in F5 and AIP (Lehmann and Scherberger, 2013), although more so in AIP. Therefore, some similarities between the behavioral and neural results of the current study and previous reaching studies are expected.

Not all methods achieved the same level of performance overall $(p<0.001$, Kruskal-Wallis ANOVA). The best method on average, SCAR, was able to explain $18 \%$ of the variance in RT in F5 and $6 \%$ in AIP. The SCAR method and the projection (before go cue) method performed best in F5 for animals B and Z, explaining 18 and $16 \%$ of the variance in RT, respectively. In animal $\mathrm{S}$, this pattern differed in F5, because the best performing methods were SCAR and Euclidean distance (after go cue), explaining 17 and $13 \%$ of variance in RT, respectively. The mean RT prediction for each animal is summarized in Figure 6A. There was no effect of grip type ( $p=0.69$, Kruskal-Wallis ANOVA) in all animals, suggesting that RT could be predicted equally well regardless of grip. Additionally, there was no effect of crossvalidation fold ( $p=0.93$, Kruskal-Wallis ANOVA), confirming that segregating the data into training and testing trials did not introduce inconsistencies into the results.

As described previously, Task 2 contained different task types (instructed, free choice, and delayed instructed). There was a 


\section{A}

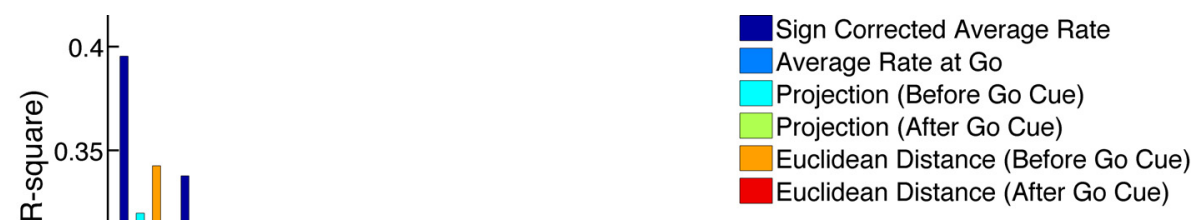


A

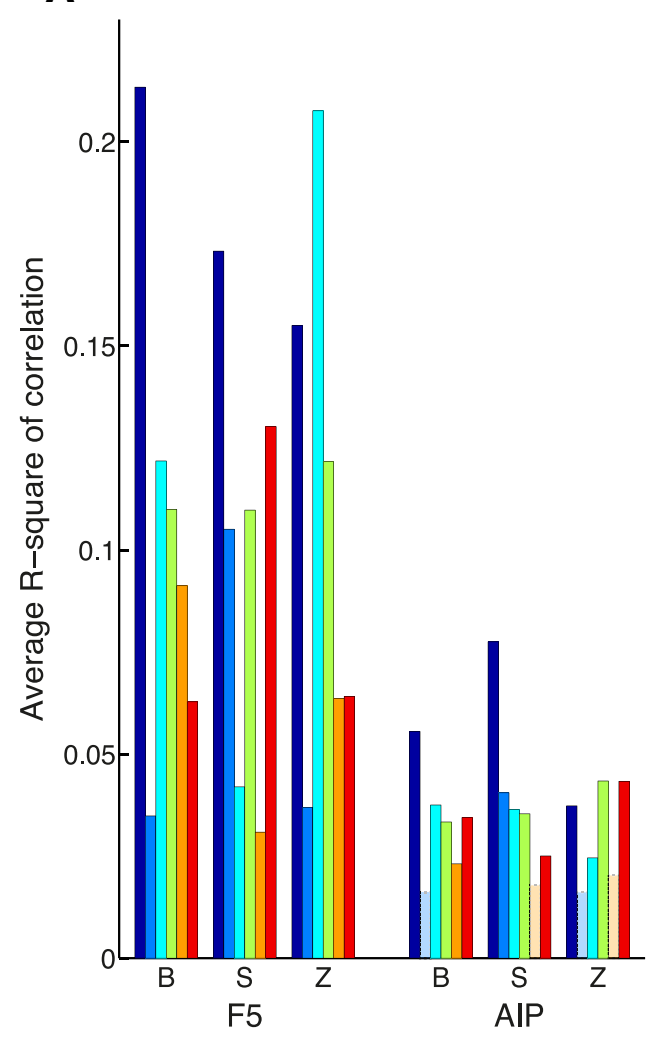

C

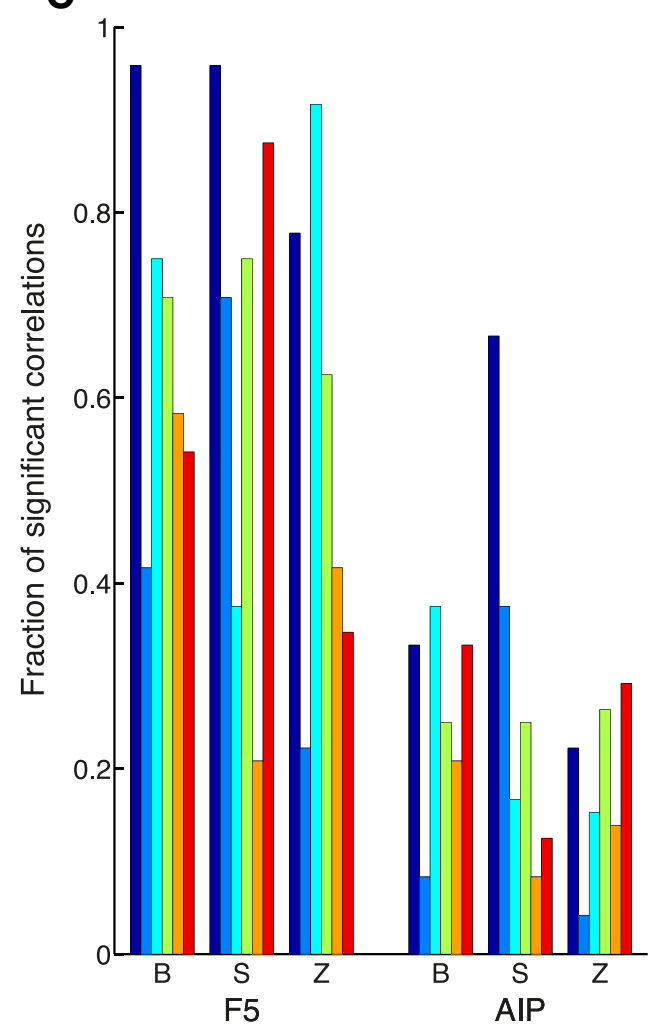

B
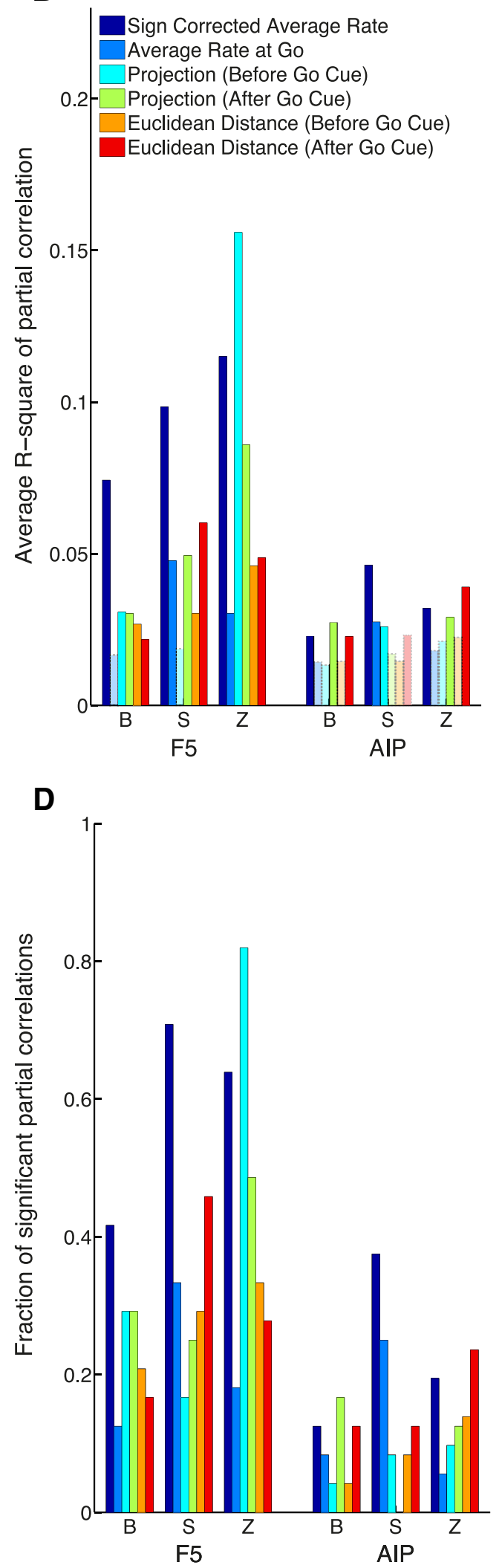

Figure 6. Comparison of prediction performance and fraction of significant full/partial correlations between predictors and RT over all task conditions, datasets, and brain areas. $A$, Average fraction of RT variance explained by correlation. $\boldsymbol{B}$, Average fraction of RT variance explained by partial correlation. Significant results are illustrated as solid bars, whereas the open bars show results that can be explained by chance $(p \geq 0.01)$. C, Fraction of conditions with significant correlations $(p<0.05)$. D, Fraction of conditions with significant partial correlations $(p<0.05)$. 
fit a set of weights to all units (MATLAB function regress). The results of this analysis, when cross-validated identically to the main analysis (twofold), show that a linear regression over all individual units can explain 3.9\% of variance in RT in F5 and $2.2 \%$ in AIP, with a significant advantage of F5 over AIP $(p<$ 0.001, Kruskal-Wallis ANOVA). However, this performance is only one-quarter of the projection or SCAR methods overall. Because the number of available units frequently outnumbers the number of available trials, coefficients cannot be ideally identified. For this reason, the regression often excluded up to $15 \%$ of the units in each dataset by assigning them a coefficient of zero.

To deal with the small number of trials available, it is also possible to use stepwise linear regression to add or remove units based on how their inclusion affects the model (MATLAB function stepwisefit). To produce an optimal solution, the model was initialized with only a constant term and units were subsequently added if they significantly improved the model ( $F$ statistic, $p<$ $0.05)$. The results of this analysis show that a stepwise linear regression over all individual units can explain $12.1 \%$ of variance in RT in F5 and 3.4\% in AIP, with a significant advantage of F5 over AIP $(p<0.001$, Kruskal-Wallis ANOVA). However, in this analysis, between 77 and $97 \%$ of units were excluded from the model to produce an optimal fit. Together, the linear regression results are consistent with previous analyses showing an advantage of F5 over AIP and are similar to the results obtained by selecting units by variance over trials (see Fig. 8). However, their usefulness is limited, at least in datasets with a restricted number of trials.

\section{Removing the effect of the memory period does not eliminate RT prediction}

The length of the memory period was strongly negatively correlated with RT in all tasks and animals (Fig. 2). To ensure that a straightforward encoding of the memory period in the firing rates of individual units was not responsible for our findings, all methods were retested using partial correlation. As described in Materials and Methods, partial correlation allows for the correlation of two variables while controlling for the linear effects of one or more additional variables. Here we controlled for the effect of memory period length on RT. Figure $6 B$ shows the mean $R^{2}$ over all datasets while controlling for the effect of memory period length. Partial correlation reduces the performance of all methods, but almost all methods remain above chance level in F5. In AIP, all methods are reduced to chance level in at least one animal, with the exception of the SCAR method. The largest reduction in performance caused by partial correlation was $66 \%$ over all methods in animal B, suggesting a strong reliance on the memory period length and consistent with the unexpected direction of the shift in the correlation coefficient distribution of animal B in Figure $4 C$. The smallest reduction in performance was $25 \%$ in animal Z. In AIP, results of each animal were never reduced by $>38 \%$. For comparison, the mean $R^{2}$ using the standard correlation metric is shown in Figure 6A. Similarly, the number of significant correlations was reduced when using partial correlation as illustrated in Figure 6D.

\section{Anterior AIP outperforms posterior AIP}

A number of recent studies have highlighted that the anterior (aAIP) and posterior ( $\mathrm{pAIP}$ ) subdivisions of AIP differentially encode visual task parameters (Baumann et al., 2009; Romero and Janssen, 2014) and differ drastically in their effective connectivity (Premereur et al., 2015). Because it is not well understood how these two areas differ in their contribution to preparatory activity for grasping, we further segregated our units into aAIP and pAIP corresponding to the ante- rior and posterior implanted arrays, respectively, and repeated the main analyses.

Unlike the comparison between F5 and AIP, the number of units recorded on each array within AIP differed significantly for all animals ( $p<0.05$, Wilcoxon's signed-rank test). Therefore, for each dataset, units were discarded randomly from the larger set until an equal number of units were present from each subarea (stratification).

If the same RT prediction methods used in the main analysis are applied to subdivisions of AIP, there is a small, but significant, advantage of aAIP over pAIP $(p=0.021$, Kruskal-Wallis ANOVA). Most of this advantage comes from the projection (pre-go) method, with an average $R^{2}$ of 0.031 in aAIP and 0.019 in pAIP ( $p<0.01$, Wilcoxon's signed-rank test). In agreement with the main results, there was no significant difference in RT prediction between grip conditions or cross-validation folds ( $p=$ 0.36 and $p=0.86$, Kruskal-Wallis ANOVA). These findings are in line with the emerging view that a gradient of visual to motor processing exists between pAIP and aAIP.

\section{Multiple regression does not improve RT prediction}

By combining multiple prediction methods in a multiple regression, it is possible to capitalize on the potential orthogonality between different predictors. To test whether a multiple regression could increase overall prediction of RT, we first replicated the regression described by Afshar et al. (2011), which consists of a regression of the pre-go and post-go cue versions of the projection and the velocity projection methods. Because the velocity projection method performed poorly in our analysis, it was not expected for this regression to significantly improve RT prediction. In fact, this four-factor multiple regression only outperformed simpler unimodal and bimodal regressions consisting of subsets of these factors in $16.7 \%$ of all datasets in F5 and $11.1 \%$ in AIP ( $F$ test). Furthermore, this regression never achieved a lower BIC score than more parsimonious regressions in any dataset or brain area, suggesting that combining these four factors in a regression is not justified in our dataset.

A number of other regressions were tested, but in no case were $>50 \%$ of datasets in F5 and $16.7 \%$ of datasets in AIP able to significantly outperform simpler regressions ( $F$ test). Furthermore, none of these multiple factor regressions achieved a lower BIC in $>11.1 \%$ of datasets in F5 and in none of the datasets in AIP.

Because multiple regression performs best when individual variables are independent, it would be unlikely to explain significantly more variance in RT if our predictors are highly correlated. In fact, most methods are highly correlated with one another in our dataset (minimum $R^{2}>0.14$ ), with the exception of the velocity projection method $\left(R^{2}<0.03\right)$, which performed poorly in the main analysis.

\section{No alternative reference point can outperform SCAR}

The SCAR method relies on first correlating the firing rate of each unit with RT and then inverting based on the resulting correlation coefficient. Because this method relies on cross-validation, it would be preferable to perform a method that does not rely on previous information. To ensure that this alternative was not possible, a control was performed. The mean firing rate at multiple time points (up to $2 \mathrm{~s}$ ) before the go cue was subtracted from the firing rate of each single trial, and the absolute value of the resulting signal was taken. Subsequently, the firing rate on each trial was averaged over units and correlated with RT. This method has the effect of inverting the activity of each unit relative to the mean firing rate at some previous time point. In no case 
A

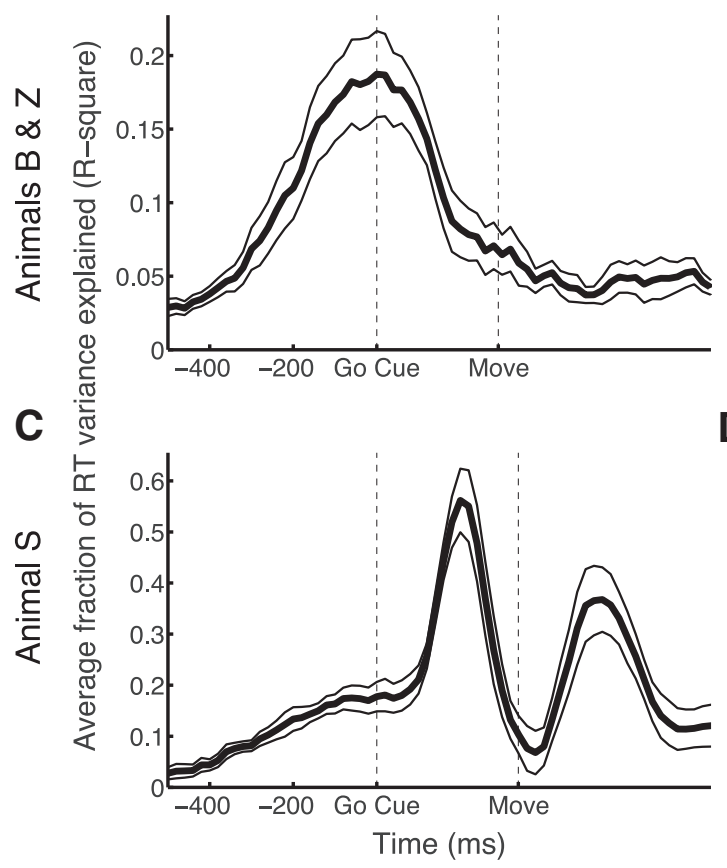

B

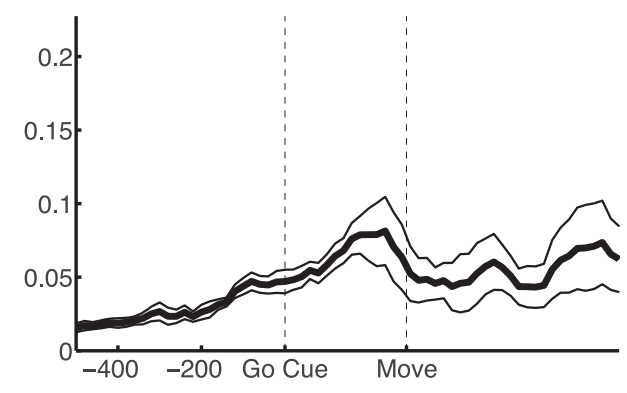

D

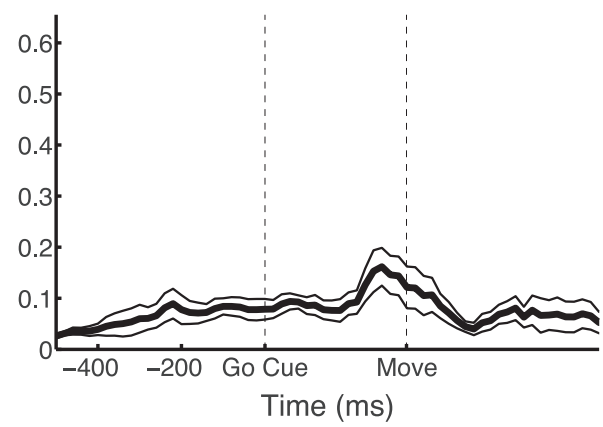

Figure 7. Stability of the sign-correction vector determined at the go cue by the SCAR method. RT prediction is calculated using sign-corrected neural activity around each time point. $\boldsymbol{A}, \boldsymbol{B}, \mathrm{SCAR}$ method as a function of time for animals B and Z in areas F5 (A) and AIP $(\boldsymbol{B}) . \boldsymbol{C}, \boldsymbol{D}$, SCAR method as a function of time for animal $S$ in areas F5 (C) and AIP $(\boldsymbol{D})$. Dashed lines indicate the go cue and the median movement onset (Move). Note the difference in peak RT prediction in F5 between animals $B$ and $Z$ and animal $S$.

was such a method able to explain more or equal variance in RT than the SCAR method, suggesting that there exists no trivial alternative to pretesting each individual unit with respect to measured RT. The same was true if grip cue-aligned activity was used for reference.

\section{Consistency of the sign-correction vector during movement}

Because the SCAR method relies on previous information of the relationship between firing rate and RT, we wondered whether the learned sign-correction vector, which is used to invert the activity of positively-correlated units, revealed a natural alignment of firing rates across time or whether it simply fit the data well at the time of the go cue. To test this, we used the signcorrection vectors determined in the main analysis to repeat the SCAR method using neural activity not just from the go cue but also at variable time points from $500 \mathrm{~ms}$ before to $800 \mathrm{~ms}$ after the go cue. The results of this analysis are depicted in Figure 7. As can be seen in Figure $7 C$ for animal $S$ in F5, maximal RT prediction is achieved shortly before median movement onset $\left(R^{2}=0.56\right)$. If neural activities of many units peak shortly before movement onset, then the SCAR method should perform best at this time. Such a result would suggest that trials on which activity drifted toward the firing rate observed at movement onset were more likely to be trials with a short RT, in line with the rise-to-threshold hypothesis (Erlhagen and Schöner, 2002).

However, as can be seen in Figure $7 A$, the results of animals $B$ and $\mathrm{Z}$ differed significantly in F5. In this case, the peak RT prediction occurs precisely around the time of go cue. In contrast to the results of animal S, a peak at the go cue suggests that, although our sign correction was able to properly align the firing of each unit at the go cue, it does not necessarily represent a consistent pattern in the firing of the underlying units.

In AIP, peak RT prediction was achieved in all animals shortly before median movement onset, i.e., a higher (sign-corrected) firing rate on single trials tended to lead to shorter RTs. Maximal RT prediction before movement onset can be explained by the idea that activity either rises during the memory period to achieve peak activity during the movement or that activity is decreased during the memory period to reach a minimum during movement. This result in AIP is consistent with a study showing significant RT prediction from activity in AIP shortly before movement onset (Verhoef et al., 2015).

\section{Variance selection allows high performance with a subset of units}

Because all recorded units were included in the previous analysis, we were curious whether a subset of units could be selected that performed equally well or better than the entire population. To test this, a variance selection of units was performed. Units were discarded from the analysis in order of increasing variance in spike count (at the go cue) across trials. This way, units with higher variances were preferentially included. For the two best performing methods, SCAR and projection (before go cue), the variance selection performed significantly better than chance ( $p<0.05$, Bonferroni's corrected) in F5 for all animals (Fig. $8 A, C)$. In AIP, only variance selection using the SCAR method outperformed chance (Fig. $8 B, D$ ).

In all cases, selecting units by variance did not improve maximal performance, as expected. In fact, when comparing performance using all units to a smaller subset in F5, using a variance-selected subset of only 32 or $18 \%$ of recorded units, for the SCAR and projection (before go cue) methods, respectively, suffered only a $5 \%$ decrease in performance. For the SCAR method, it was only necessary to use a subset of $23 \%$ of the available units in F5 to attain 95\% of maximal performance. Together, these results suggest that, when units are selected by variance at the go cue, only relatively small subsets of the recorded units are required to attain virtually maximal performance. More impor- 
A

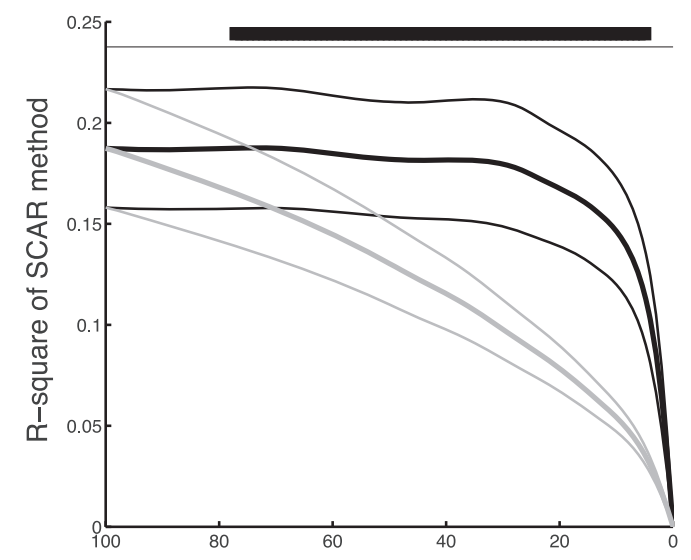

C

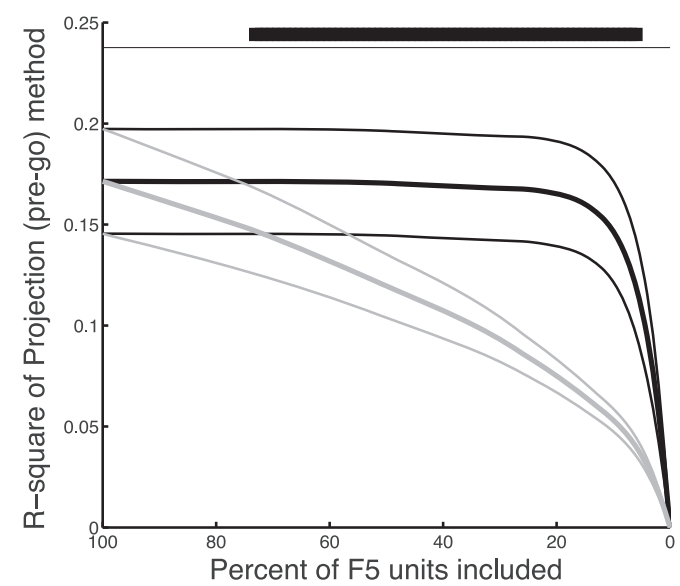

B

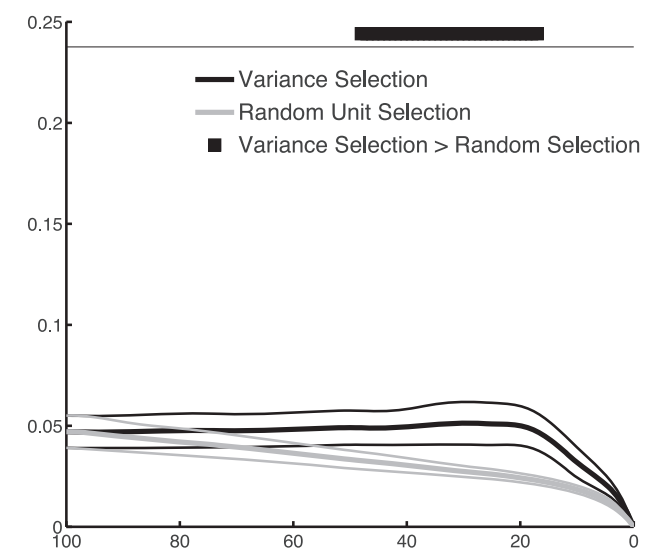

D

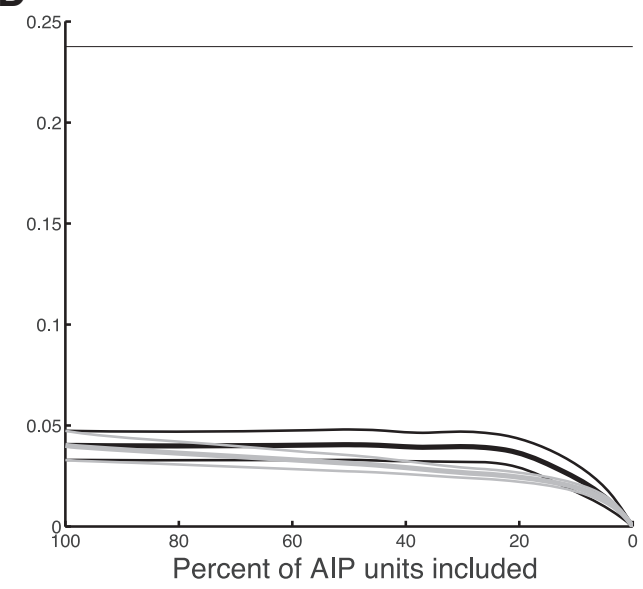

Figure 8. Selection of units by firing rate variance at the go cue for the two best performing methods in animals $B$ and $Z$. $A, B, V$ ariance selection of units versus random selection using the $S C A R$ method in F5 (A) and AIP $(\boldsymbol{B}) . \boldsymbol{C}, \boldsymbol{D}$, Variance selection of units versus random selection using the projection (before go cue) method in F5 (C) and AIP (D). Horizontal black bars on top represent unit percentages in which the variance selection performed significantly better than random selection ( $p=0.05$, Bonferroni's corrected, permutation test).

tantly, including all units in the population does not appear to add noise to these methods, because maximal performance is achieved when including all units, suggesting that they properly describe the relationship between preparatory activity and RT at the population or network level.

\section{Variability of RT axis from day to day and animal to animal}

When considering each dataset separately, it became clear that the day-to-day variability in RT prediction for each method is relatively high (Fig. 5). To elucidate how neural trajectories, which are presumably very similar over sessions, could explain very different amounts of variance in RT, we visualized a few individual sessions using PCA. This second dimensionality reduction method was introduced, in addition to the GPFA used in Figure $2 A$, to visualize average trajectories as opposed to single trials. In contrast to GPFA, which applies many different and sometimes large smoothing kernels, PCA allows more direct control over the amount of smoothing over time. In Figure 9, the first two principal components of individual conditions of individual recording sessions are shown. The mean trajectory over all trials is depicted along with the mean trajectory of trials binned into slow, medium, and fast RT trials. In every subplot, a visualization of the projection (after go cue) method is presented from the data. In this visualization, the position of single trials along the dashed projection axis would determine our measurement for how far along the mean neural trajectory this trial is. The subsequent length of the projection of each single trial onto this axis would then be used to predict RT. In Figure 9A, trajectories of a power grip condition are shown from dataset B140509. It appears that the fast and slow RT trials are located distantly to each other along the projection axis, suggesting that this axis would be valuable in explaining trial-to-trial RT variability. This was in fact the case, because the projection (after go cue) method was able to explain $27 \%$ of the variance in RT in the main analysis of this dataset. However, note that the position of the fastest RT trials is less far along the mean trajectory than slow trials, directly contradicting the predictions of our hypothesis. As we noted in Figure $6 B$, much of the RT prediction obtained in animal B was eliminated by controlling for the effect of memory period length. Based on the trajectory in Figure 9A, it seems that trials with longer memory periods tended to continue along the projection axis instead of lingering near the mean trajectory. Because longer memory periods led to slower RTs for the most extreme memory period lengths $(1300 \mathrm{~ms})$, trials that have progressed farther along the mean indicated slower RT trials.

Plotted in Figure $9 B$ is the mean trajectory of the precision grip on the very next dataset (B140515). The mean trajectory for this condition is very similar to that of Figure $9 A$. However, the orientation of the projection axis is approximately orthogonal to that of an axis running through the slow and fast RT trials, sug- 

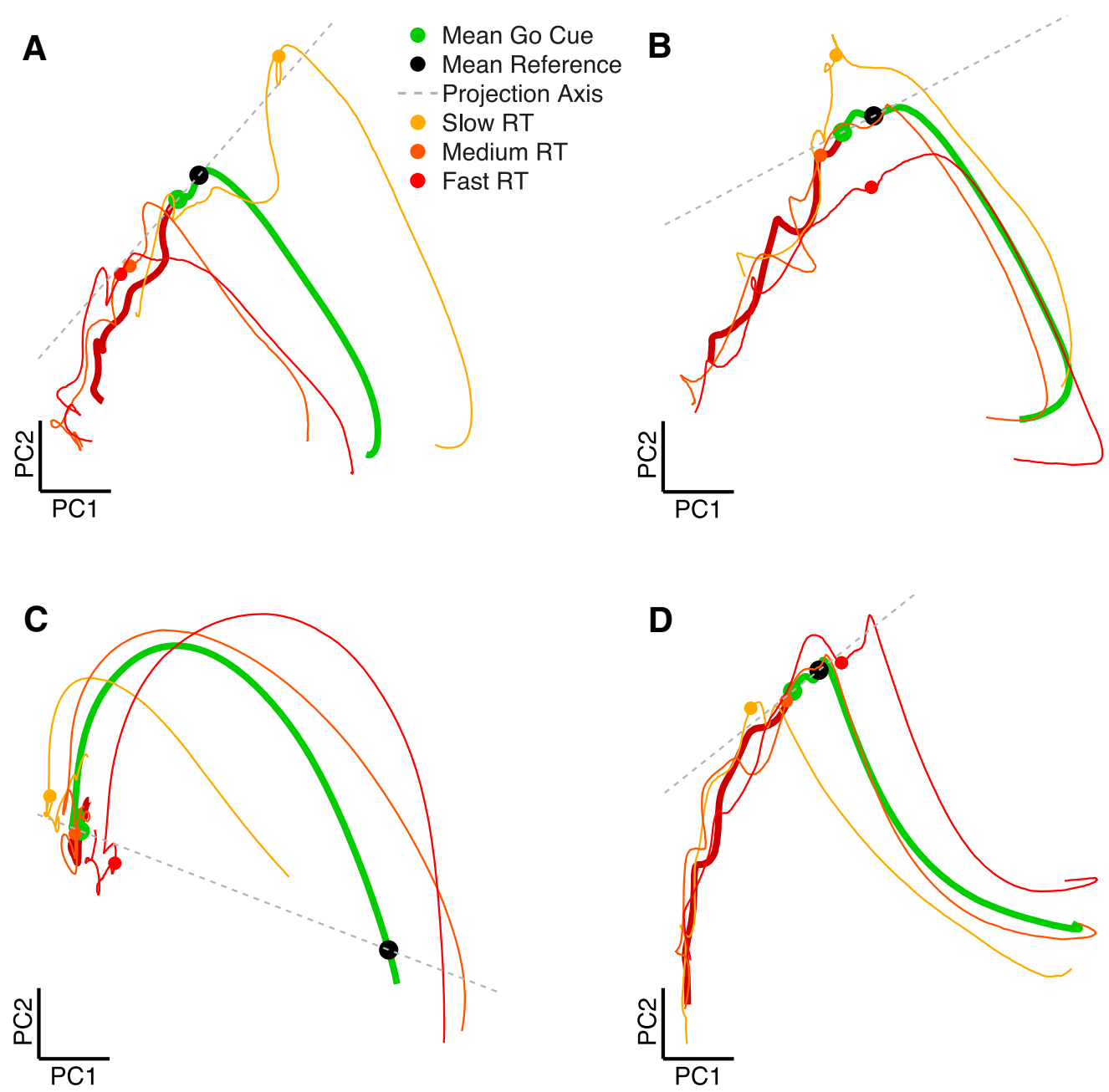

Figure 9. Low-dimensional representation (PCA) of neural state space illustrating day-to-day and animal-to-animal variability. Trajectories are plotted in the two principal components of area F5 explaining the most variance. $\boldsymbol{A}$, Trajectory of a power grip from dataset B140509 in which RT is most variable along the projection axis. $\boldsymbol{B}$, Trajectory of a precision grip from dataset B140515 in which RT is most variable orthogonal to the projection axis. C, Trajectory of a precision grip from dataset $S 120913$ in which RT is most variable on the shared distance and projection axis. $\boldsymbol{D}$, Trajectory of a power grip from dataset Z120921 in which RT is most variable along the projection axis.

gesting that this axis would explain only a small amount of variability in trial-to-trial RT. The projection (after go cue) method performed at chance level for this dataset, only explaining $3 \%$ of the variance in RT. Therefore, it seems plausible that, even when trajectories are similar, it is possible for RT variance to be rotated relative to the mean trajectory, suggesting that the mean trajectory may not always be an ideal reference. In this case, the Euclidean distance (after go cue) method performed significantly better because trials with shorter RT were located closer to the movement onset state in the state space.

The trajectory in Figure $9 C$ shows a precision grip from dataset S1209013. It seems to differ substantially from the other trajectories. In this case, there is no clear progression of the preparatory trajectory near the go cue, and there is also no abrupt change in the directionality of the trajectory after the go cue. Therefore, it is not surprising that both projection methods performed quite poorly on this dataset. Only by selecting a $\Delta t$ for the projection (after go cue) method that was quite large $(210 \mathrm{~ms})$ could improve RT prediction. In this case, the projection (after go cue) and Euclidean distance (after go cue) methods performed similarly, which is not surprising because projection and distance become mathematically similar for large $\Delta t$ values.
Figure $9 D$ represents an ideal trajectory of a power grip from dataset Z120921. In this case, trials that are farther along the projection axis correspond to trials with shorter RTs, in line with the predictions of the projection method.

\section{Discussion}

Using simultaneous neural recordings from three animals, we have shown that preparatory activity in both premotor and parietal cortices is correlated with trial-to-trial variability in RT. However, the activity in F5 is far more predictive of RT than in AIP. Although the length of the memory period facilitated RT predictability, our findings cannot be explained purely based on this relationship. The use of a state space framework, made possible by the parallel recording of many units, represents a major step forward in understanding the relationship between preparatory activity and behavioral parameters.

\section{Trial-to-trial RT prediction}

Although response characteristics and tuning properties of AIP and F5 neurons can be very similar (Baumann et al., 2009; Fluet et al., 2010), we have shown that their trial-to-trial relationship with RT differs greatly (Fig. 5). The current result is not trivial, because F5 and 
AIP are densely and reciprocally connected (Luppino et al., 1999; Borra et al., 2008). However, the level of RT predictability found in the preparatory activity of AIP units is consistent with previous studies in nearby areas (Janssen and Shadlen, 2005; Snyder et al., 2006).

Together, the relative advantage of F5 over AIP is not altogether surprising given the fact that $\mathrm{F} 5$, which has projections to the spinal cord (He et al., 1993; Borra et al., 2010) and a facilitation effect on M1 (Shimazu et al., 2004), must naturally be involved in the transition between preparation and movement execution.

\section{Comparing prediction methods}

High performance of the projection method, matched only by the SCAR method, is consistent with the "initial conditions" hypothesis formulated by Afshar et al. (2011) from activity in PMd. Even after controlling for the effect of memory period length, the projection method still performs above chance level; however, the SCAR method can outperform the projection method, especially in Task 1. SCAR offers an alternative explanation for the relationship between single-unit firing and RT. In this framework, most individual units have a consistent relationship with RT, i.e., higher or lower firing rates before the go cue are associated with shorter RT. Controlling for the sign of this relationship was able to increase RT prediction up to four times and follows well from the observation that the preparatory activity in PMd is both positively and negatively correlated with RT (Riehle and Requin, 1993), as in F5.

Could subpopulations of these units explain prediction of RT? If this were the case, we would expect units that fire more during the delay would continue to rise during the movement. In two of three animals, the SCAR method peaked in RT prediction at the go cue, suggesting that this sign correction was a local property and not a consistent property of each unit (Fig. $7 A, C$ ). Indeed, activity is often higher in the delay period than during the movement (Crammond and Kalaska, 2000), suggesting that the relationship between firing during preparation and movement is complex (Churchland and Shenoy, 2007b).

Interestingly, maximal performance is always achieved for the projection and SCAR methods when including all recorded units and not a variance-selected subset in F5 (Fig. 8), supporting the conclusion that both methods accurately describe populationlevel features and are not simply dominated by specific subpopulations of units.

Previously, the best performing method was a multiple regression of projection and velocity projection components (Afshar et al., 2011). We did not find significant performance of the velocity projection method or any multiple regression. When examining our neural trajectories, it seems that in many cases the speed of change in neural signal remained high or even increased during the memory period, especially in Task 1 (our unpublished data). This may represent an interesting quality of $\mathrm{F} 5$ activity that differs from activity in PMd. Such memory-related activity could mask relationships between trial-to-trial neural velocity and RT, especially after factoring in the length of the memory period.

\section{Differences between PMv and PMd}

To our knowledge, the preparatory activity recorded in $\mathrm{F} 5$ in our study explains more trial-to-trial variance in RT than any other published study. However, the results obtained in nearby PMd are quantitatively comparable (Afshar et al., 2011). A number of studies have systematically contrasted PMv and PMd (for review, see Hoshi and Tanji, 2007). It may be that F5 is more involved in the specific timing and execution of reaching movements than PMd, as evidenced by chemical inactivation (Kurata and Hoff- man, 1994). Nevertheless, both PMv and PMd are essential for grasping movements (Raos et al., 2004, 2006). Electrical microstimulation in PMd during preparation (Churchland and Shenoy, 2007a), and potentially F5 (Gerits et al., 2012), delays movement onset.

Although PMd and PMv are part of relatively distinct parietofrontal networks, they both have an important effect on behavioral timing. Additionally, both PMd and PMv project to similar locations within M1 and lack a clear hierarchy (Dum and Strick, 2005), suggesting that their roles are complementary and not sequential.

\section{Limitations}

To rule out premature muscle contractions as an explanation for RT prediction obtained during a delay, electromyographic recording of relevant muscles has been used in the past (Churchland et al., 2006c; Afshar et al., 2011). Such recordings were not undertaken in the current study; however, we do not believe that premature muscle contractions are a likely cause of the RT prediction observed here for three reasons. First, the hands of all animals remained completely still on the hand rest buttons until after the go cue had been given, as confirmed by infrared monitoring. Second, the RTs of all animals were well above $200 \mathrm{~ms}$ in at least $97 \%$ of trials, suggesting that they appropriately awaited the go cue. Third, animals successfully withheld movement during the catch trials, suggesting that they were properly awaiting the go signal.

Although the primary interest of the current study was grasping actions, all movements included a large reaching component as well. It remains a possibility that the relative advantage of F5 over AIP could in part be attributable to a larger role of F5 in reaching than AIP. However, previous studies dissociating reaching and grasping have shown that PMv is greatly involved in the representation of grasping without a reach component (HeppReymond et al., 1994) and is potentially even less involved in reach encoding than AIP (Lehmann and Scherberger, 2013). Therefore, finding higher RT prediction accuracy in F5 rather suggests a larger influence of the grasping component in the neural signal.

\section{Implications for models of motor preparation}

It is clear that the most dominant factor in the neural trajectories of animal B is the length of the memory period itself (Fig. 9A), which seems to act counter to the notion of an optimal subspace, because trials do not congregate within an area of low variability. It has been shown that variability is decreased by external stimuli, which was observed in PMd (Churchland et al., 2006c) and a number of other cortical areas (Churchland et al., 2010). If F5 neurons were multiplexing many factors in addition to a motor plan such as anticipation of the go cue, similar to hazard rate (Janssen and Shadlen, 2005), or variability in attention over longer periods of time, trial-to-trial variability might be increased at go cue. Furthermore, encoding of the length of the memory period clearly increased RT predictability in F5 and AIP, as evidenced by the decrease in predictability when using partial correlation. Additional work is needed to determine the extent to which F5 and AIP encode cue anticipation or attention-related factors.

Alternatively, it could be that the subspace required to successfully complete the grasping movement is sufficiently large to allow trajectories to lie in a relatively wide space. The absence of a static prepare-and-hold state is consistent with the augmented view of the initial conditions hypothesis posited by Ames et al. 
(2014), who showed that the memory state is bypassed in PMd when time to prepare an action is lacking. In this view, the subspace required to successfully complete an action, i.e., with no penalty in movement generation, but a possible penalty in RT, could be quite broad. However, it is clear that F5 firing rates do not necessarily congregate in a specific part of the state space given enough time, as would be predicted by an attractor model of preparatory dynamics. The interesting question of determining whether such a prepare-and-hold state is necessary in F5 or AIP, along with whether the observed preparatory processes set the initial conditions of a dynamical system, as they do in PMd and M1 (Churchland et al., 2012; for review, see Shenoy et al., 2013), are left to future works.

Recently, the ability to record activity from many neurons simultaneously has opened up new possibilities in the investigation of the motor and premotor cortices (for review, see Churchland et al., 2007). The current study explores the relationship between preparatory activity in large populations of neurons and subsequent behavior, shedding light on the differential role of parietal and frontal cortices in this process.

\section{References}

Afshar A, Santhanam G, Yu BM, Ryu SI, Sahani M, Shenoy KV (2011) Single-trial neural correlates of arm movement preparation. Neuron 71: 555-564. CrossRef Medline

Ames KC, Ryu SI, Shenoy KV (2014) Neural dynamics of reaching following incorrect or absent motor preparation. Neuron 81:438-451. CrossRef Medline

Baumann MA, Fluet MC, Scherberger H (2009) Context-specific grasp movement representation in the macaque anterior intraparietal area. J Neurosci 29:6436-6448. CrossRef Medline

Borra E, Belmalih A, Calzavara R, Gerbella M, Murata A, Rozzi S, Luppino G (2008) Cortical connections of the macaque anterior intraparietal (AIP) area. Cereb Cortex 18:1094-1111. CrossRef Medline

Borra E, Belmalih A, Gerbella M, Rozzi S, Luppino G (2010) Projections of the hand field of the macaque ventral premotor area F5 to the brainstem and spinal cord. J Comp Neurol 518:2570-2591. CrossRef Medline

Carpaneto J, Umiltà MA, Fogassi L, Murata A, Gallese V, Micera S, Raos V (2011) Decoding the activity of grasping neurons recorded from the ventral premotor area F5 of the macaque monkey. Neuroscience 188:80-94. CrossRef Medline

Churchland MM (2015) Using the precision of the primate to study the origins of movement variability. Neuroscience 296:92-100. CrossRef Medline

Churchland MM, Shenoy KV (2007a) Delay of movement caused by disruption of cortical preparatory activity. J Neurophysiol 97:348-359. CrossRef Medline

Churchland MM, Shenoy KV (2007b) Temporal complexity and heterogeneity of single-neuron activity in premotor and motor cortex. J Neurophysiol 97:4235-4257. CrossRef Medline

Churchland MM, Afshar A, Shenoy KV (2006a) A central source of movement variability. Neuron 52:1085-1096. CrossRef Medline

Churchland MM, Santhanam G, Shenoy KV (2006b) Preparatory activity in premotor and motor cortex reflects the speed of the upcoming reach. J Neurophysiol 96:3130-3146. CrossRef Medline

Churchland MM, Yu BM, Ryu SI, Santhanam G, Shenoy KV (2006c) Neural variability in premotor cortex provides a signature of motor preparation. J Neurosci 26:3697-3712. CrossRef Medline

Churchland MM, Yu BM, Sahani M, Shenoy KV (2007) Techniques for extracting single-trial activity patterns from large-scale neural recordings. Curr Opin Neurobiol 17:609-618. CrossRef Medline

Churchland MM, Yu BM, Cunningham JP, Sugrue LP, Cohen MR, Corrado GS, Newsome WT, Clark AM, Hosseini P, Scott BB, Bradley DC, Smith MA, Kohn A, Movshon JA, Armstrong KM, Moore T, Chang SW, Snyder LH, Lisberger SG, Priebe NJ, Finn IM, Ferster D, Ryu SI, Santhanam G, Sahani M, Shenoy KV (2010) Stimulus onset quenches neural variability: a widespread cortical phenomenon. Nat Neurosci 13:369-378. CrossRef Medline

Churchland MM, Cunningham JP, Kaufman MT, Foster JD, Nuyujukian P,
Ryu SI, Shenoy KV (2012) Neural population dynamics during reaching. Nature 487:51-56. CrossRef Medline

Cramér H (1946) Mathematical methods of statistics. Princeton: Princeton UP.

Crammond DJ, Kalaska JF (2000) Prior information in motor and premotor cortex: activity during the delay period and effect on pre-movement activity. J Neurophysiol 84:986-1005. Medline

Dum RP, Strick PL (2005) Frontal lobe inputs to the digit representations of the motor areas on the lateral surface of the hemisphere. J Neurosci 25 : 1375-1386. CrossRef Medline

Erlhagen W, Schöner G (2002) Dynamic field theory of movement preparation. Psychol Rev 109:545-572. CrossRef Medline

Fetz EE (1992) Are movement parameters recognizably coded in the activity of single neurons? Behav Brain Sci 15:679-690.

Fluet MC, Baumann MA, Scherberger H (2010) Context-specific grasp movement representation in macaque ventral premotor cortex. J Neurosci 30:15175-15184. CrossRef Medline

Gerits A, Farivar R, Rosen BR, Wald LL, Boyden ES, Vanduffel W (2012) Optogenetically induced behavioral and functional network changes in primates. Curr Biol 22:1722-1726. CrossRef Medline

Ghez C, Favilla M, Ghilardi MF, Gordon J, Bermejo R, Pullman S (1997) Discrete and continuous planning of hand movements and isometric force trajectories. Exp Brain Res 115:217-233. CrossRef Medline

Gozani SN, Miller JP (1994) Optimal discrimination and classification of neuronal action potential waveforms from multiunit, multichannel recordings using software-based linear filters. IEEE Trans Biomed Eng 41: 358-372. CrossRef Medline

Hanes DP, Schall JD (1996) Neural control of voluntary movement initiation. Science 274:427-430. CrossRef Medline

He SQ, Dum RP, Strick PL (1993) Topographic organization of corticospinal projections from the frontal lobe: motor areas on the lateral surface of the hemisphere. J Neurosci 13:952-980. Medline

Hepp-Reymond MC, Hüsler EJ, Maier MA, Q1 HX (1994) Force-related neuronal activity in two regions of the primate ventral premotor cortex. Can J Physiol Pharmacol 72:571-579. CrossRef Medline

Hoshi E, Tanji J (2007) Distinctions between dorsal and ventral premotor areas: anatomical connectivity and functional properties. Curr Opin Neurobiol 17:234-242. CrossRef Medline

Janssen P, Shadlen MN (2005) A representation of the hazard rate of elapsed time in macaque area LIP. Nat Neurosci 8:234-241. CrossRef Medline

Kakei S, Hoffman DS, Strick PL (2001) Direction of action is represented in the ventral premotor cortex. Nat Neurosci 4:1020-1025. CrossRef Medline

Kraskov A, Dancause N, Quallo MM, Shepherd S, Lemon RN (2009) Corticospinal neurons in macaque ventral premotor cortex with mirror properties: a potential mechanism for action suppression? Neuron 64: 922-930. CrossRef Medline

Kurata K, Hoffman DS (1994) Differential effects of muscimol microinjection into dorsal and ventral aspects of the premotor cortex of monkeys. J Neurophysiol 71:1151-1164. Medline

Kutas M, Donchin E (1974) Studies of squeezing: handedness, responding hand, response force, and asymmetry of readiness potential. Science 186 : 545-548. CrossRef Medline

Lehmann SJ, Scherberger H (2013) Reach and gaze representations in macaque parietal and premotor grasp areas. J Neurosci 33:7038-7049. CrossRef Medline

Luppino G, Murata A, Govoni P, Matelli M (1999) Largely segregated parietofrontal connections linking rostral intraparietal cortex (areas AIP and VIP) and the ventral premotor cortex (areas F5 and F4). Exp Brain Res 128:181-187. CrossRef Medline

McQuarrie AD, Tsai CL (1998) Regression and time series model selection. Hackensack, NJ: World Scientific.

Murata A, Fadiga L, Fogassi L, Gallese V, Raos V, Rizzolatti G (1997) Object representation in the ventral premotor cortex (area F5) of the monkey. J Neurophysiol 78:2226-2230. Medline

Murata A, Gallese V, Luppino G, Kaseda M, Sakata H (2000) Selectivity for the shape, size, and orientation of objects for grasping in neurons of monkey parietal area AIP. J Neurophysiol 83:2580-2601. Medline

Musial PG, Baker SN, Gerstein GL, King EA, Keating JG (2002) Signal-tonoise ratio improvement in multiple electrode recording. J Neurosci Methods 115:29-43. CrossRef Medline

National Research Council (2003) Guidelines for the care and use of mam- 
mals in neuroscience and behavioral research. Washington, DC: National Academies Press.

Premereur E, Van Dromme IC, Romero MC, Vanduffel W, Janssen P (2015) Effective connectivity of depth-structure-selective patches in the lateral bank of the macaque intraparietal sulcus. PLos Biol 13:e1002072. CrossRef Medline

Quiroga RQ, Nadasdy Z, Ben-Shaul Y (2004) Unsupervised spike detection and sorting with wavelets and superparamagnetic clustering. Neural Comput 16:1661-1687. CrossRef Medline

Raos V, Umiltá MA, Gallese V, Fogassi L (2004) Functional properties of grasping-related neurons in the dorsal premotor area $\mathrm{F} 2$ of the macaque monkey. J Neurophysiol 92:1990-2002. CrossRef Medline

Raos V, Umiltá MA, Murata A, Fogassi L, Gallese V (2006) Functional properties of grasping-related neurons in the ventral premotor area F5 of the macaque monkey. J Neurophysiol 95:709-729. Medline

Riehle A, Requin J (1989) Monkey primary motor and premotor cortex: single-cell activity related to prior information about direction and extent of an intended movement. J Neurophysiol 61:534-549. Medline

Riehle A, Requin J (1993) The predictive value for performance speed of preparatory changes in neuronal activity of the monkey motor and premotor cortex. Behav Brain Res 53:35-49. CrossRef Medline

Romero MC, Pani P, Janssen P (2014) Coding of shape features in the macaque anterior intraparietal area. J Neurosci 34:4006-4021. CrossRef Medline

Rosenbaum DA (1980) Human movement initiation: specification of arm, direction, and extent. J Exp Psychol Gen 109:444-474. CrossRef Medline
Schaffelhofer S, Agudelo-Toro A, Scherberger H (2015) Decoding a wide range of hand configurations from macaque motor, premotor, and parietal cortices. J Neurosci 35:1068-1081. CrossRef Medline

Shenoy KV, Sahani M, Churchland MM (2013) Cortical control of arm movements: a dynamical systems perspective. Annu Rev Neurosci 36: 337-359. CrossRef Medline

Shimazu H, Maier MA, Cerri G, Kirkwood PA, Lemon RN (2004) Macaque ventral premotor cortex exerts powerful facilitation of motor cortex outputs to upper limb motoneurons. J Neurosci 24:1200-1211. CrossRef Medline

Snyder LH, Dickinson AR, Calton JL (2006) Preparatory delay activity in the monkey parietal reach region predicts reach reaction times. J Neurosci 26:10091-10099. CrossRef Medline

Townsend BR, Subasi E, Scherberger H (2011) Grasp movement decoding from premotor and parietal cortex. J Neurosci 31:14386-14398. CrossRef Medline

Verhoef BE, Michelet P, Vogels R, Janssen P (2015) Choice-related activity in the anterior intraparietal area during 3-D structure categorization. J Cogn Neurosci 27:1104-1115. CrossRef Medline

Wise SP (1985) The primate premotor cortex: past, present, and preparatory. Annu Rev Neurosci 8:1-19. CrossRef Medline

Yu BM, Cunningham JP, Santhanam G, Ryu SI, Shenoy KV, Sahani M (2009) Gaussian-process factor analysis for low-dimensional single-trial analysis of neural population activity. J Neurophysiol 102:614-635. CrossRef Medline 